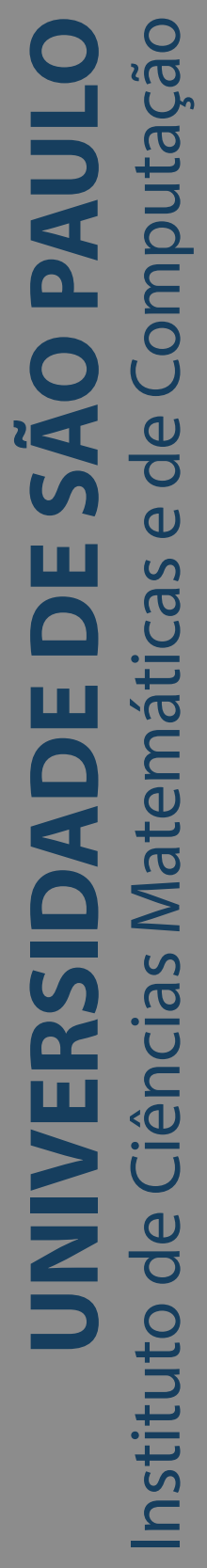

\title{
Esferas hiperbólicas com pontos cônicos
}

\section{João dos Reis Junior}

Dissertação de Mestrado do Programa de Pós-Graduação em Matemática (PPG-Mat) 

Assinatura:

\title{
João dos Reis Junior
}

\section{Esferas hiperbólicas com pontos cônicos}

\author{
Dissertação apresentada ao Instituto de Ciências \\ Matemáticas e de Computação - ICMC-USP, \\ como parte dos requisitos para obtenção do título \\ de Mestre em Ciências - Matemática. EXEMPLAR \\ $D E D E F E S A$
}

Área de Concentração: Matemática

Orientador: Prof. Dr. Carlos H. Grossi Ferreira

\section{USP - São Carlos \\ Novembro de 2020}





\title{
João dos Reis Junior
}

\section{Hyperbolic spheres with cone singularities}

\begin{abstract}
Master dissertation submitted to the Institute of Mathematics and Computer Sciences - ICMC-USP, in partial fulfillment of the requirements for the degree of the Master Program in Mathematics. EXAMINATION BOARD PRESENTATION COPY
\end{abstract}

Concentration Area: Mathematics

Advisor: Prof. Dr. Carlos H. Grossi Ferreira

USP - São Carlos

November 2020 

Dedico este trabalho às pessoas que, mesmo tentadas pelo flerte da loucura, preferem continuar seguindo o caminho da razão e sobriedade, embora mantendo a sua loucura como um hobbie. 

Em primeiro lugar, gostaria de agradecer meus dois grandes mentores em toda minha jornada científica, Sasha Ananin e Carlos H. Grossi, pacientes orientadores que me deram o prazer de conhecer matemática pela primeira vez.

Igualmente importantes nessa jornada foram meus amigos na universidade. Dentre todos os muitos deles, valem a menção Christian V. B. Lemos, Rafael F. Pereira, Philipy V. Chiovetto e Uirá N. Matos, sendo o último meu parceiro de discussões matemáticas mais frequentes, que guardo com nostalgia.

Agradeço a minha família pela melhor educação que poderiam me dar, pelo carinho e pela confiança que sempre tiveram em mim.

Agradeço a Natane Neves, minha companheira, pela confiança, paciência, carinho e companhia.

E agradeço a todos aqueles que dedicaram suas vidas a essa nobre ciência, a invenção mais elaborada da humanidade, tornando-a este corpo de conhecimento curioso e intrincado, que tive o prazer de experienciar.

Esse trabalho foi realizado com o apoio financeiro do CNPq, processo número 131527/20185, Conselho Nacional de Desenvolvimento Científico e Tecnológico (CNPq). 

"The players listen very carefully and respectfully to all that the clever men have to say about what is to happen in the next generation. The players then wait until all the clever men are dead, and bury them nicely. They then go and do something else." (The Napoleon of Notting Hill, Gilbert K. Chesterton) 



\section{RESUMO}

JOÃO DOS REIS JR. Esferas hiperbólicas com pontos cônicos. 2020. 80 p. Dissertação (Mestrado em Ciências - Matemática) - Instituto de Ciências Matemáticas e de Computação, Universidade de São Paulo, São Carlos - SP, 2020.

A presente dissertação tem como tema principal o estudo de esferas métricas com pontos cônicos, e em especial, os espaços classificadores de tais esferas. Na primeira seção é feita uma breve exposição do tratamento de geometrias clássicas utilizando formas hermitianas, que é o framework utilizado na parte principal do texto. A seguir, é apresentada uma versão (traduzida para o português) do artigo que se originou do trabalho de colaboração do autor dessa dissertação com outros 3 coautores. O artigo foi publicado no periódico Topology and its applications, 272 (2020), 107073. O preprint que deu origem à publicação também encontra-se anexado à dissertação.

Palavras-chave: Esferas hiperbólicas, Pontos cônicos, Geometria. 



\section{ABSTRACT}

JOÃO DOS REIS JR. Hyperbolic spheres with cone singularities. 2020. 80 p. Dissertação (Mestrado em Ciências - Matemática) - Instituto de Ciências Matemáticas e de Computação, Universidade de São Paulo, São Carlos - SP, 2020.

This essay has as its main theme the study of metric spheres with cone singularities, and specially, the classifying spaces of such spheres. In the first section we present a brief exposition of the treatment using hermitian forms for classical geometries, the framework utilized in the principal part of the text. Next, it is presented a version (translated to Portuguese) of the paper originated from the collaboration work between the author and another 3 co-authors. The paper has been published in the journal Topology and its applications, 272 (2020), 107073. The preprint that gave origin to the publication is also attached to this essay.

Keywords: Hyperbolic spheres, Cone sigularities, Geometry. 

1 FUNDAMENTOS DE GEOMETRIA CLÁSSICA . . . . . . . . . . 17

$1.1 \quad$ Conceitos iniciais . . . . . . . . . . . . . 17

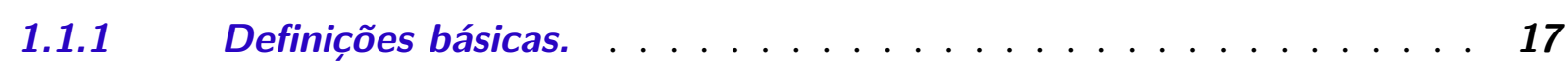

1.1.2 Algumas construções geométricas. . . . . . . . . . . . . . 19

1.1.3 Fatos preliminares. ................. 19

$1.2 \quad$ Geodésicas . . . . . . . . . . . . . . . . 20

$1.3 \quad$ Conexão de Levi-Civita . . . . . . . . . . . . . . . . 21

1.3.1 Transporte paralelo ao longo de geodésicas . . . . . . . . . . . . . 23

2 ESFÉRICAS HIPERBÓlICAS COM PONTOS CÔNICOS . . . . . . 27

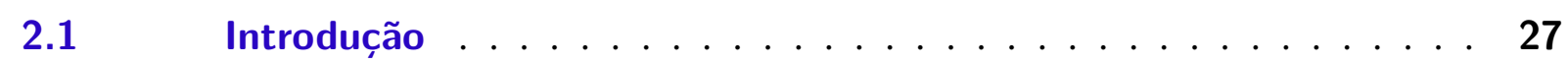

2.1.1 Caso geral . . . . . . . . . . . . . . . . . 28

2.1.2 2n-gonos hiperbólicos convexos . . . . . . . . . . . . 29

2.1.3 Geometria em $C\left(a_{0}, a_{1}, \ldots, a_{n}\right)$ e outros espaços relacionados . . . . 29

2.1.4 Breve esboço da exposição . . . . . . . . . . . . . . . 29

2.1.5 Trabalhos relacionados . . . . . . . . . . . . . . 31

$2.2 \quad$ Fatos preliminares . . . . . . . . . . . . . 31

$2.3 \quad$ Lemas técnicos . . . . . . . . . . . . . . . 35

$2.4 \quad \mathrm{O}$ espaço $S\left(a_{0}, a_{1}, a_{2}, a_{3}\right)$ de hexágonos, $\sigma=-1 \ldots \ldots$

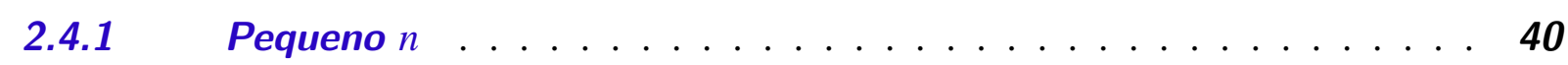

2.4.2 Topologia de $S\left(a_{0}, a_{1}, a_{2}, a_{3}\right) \ldots \ldots \ldots \ldots \ldots$

2.4.3 Discretude de $M_{3} \ldots \ldots \ldots \ldots \ldots \ldots$

$2.5 \quad$ Esferas hiperbólicas com $n+1$ pontos cônicos e $2 n$-gonos convexos 53

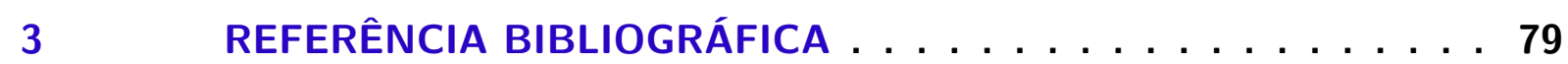





\section{FUNDAMENTOS DE GEOMETRIA CLÁSSICA}

\subsection{Conceitos iniciais}

\subsubsection{Definições básicas.}

Seja $\mathbb{K}$ um dos seguintes corpos: $\mathbb{R}$ (números reais) ou $\mathbb{C}$ (números complexos). A aplicação $a \mapsto \bar{a}$ denota a conjugação no caso $\mathbb{K}=\mathbb{C}$ e a aplicação idêntica no caso $\mathbb{K}=\mathbb{R}$.

Definição 1. Uma geometria clássica é um $\mathbb{K}$-espaço vetorial $V$ munido de uma forma hermitiana $\langle-,-\rangle$. Por definição, isto significa que a forma assume valores em $\mathbb{K}$, é biaditiva, e satisfaz as identidades $\left\langle k v_{1}, v_{2}\right\rangle=k\left\langle v_{1}, v_{2}\right\rangle \mathrm{e}\left\langle v_{1}, v_{2}\right\rangle=\overline{\left\langle v_{2}, v_{1}\right\rangle}$ para todos $v_{1}, v_{2} \in V, k \in \mathbb{K}$.

Denotamos $V^{\times}=V-\{0\}$ o espaço vetorial sem origem e $\pi: V^{\times} \rightarrow \mathbb{P}_{\mathbb{K}} V$ a projeção natural para a projetivização de $V$. O espaço tangente num ponto $p \in \mathbb{P}_{\mathbb{K}} V$ pode ser descrito como o $\mathbb{K}$-espaço vetorial

$$
\mathrm{T}_{p} \mathbb{P}_{\mathbb{K}} V=\operatorname{Lin}_{\mathbb{K}}(p, V / p)
$$

de transformações lineares de $\mathbb{K} p$ para $V / \mathbb{K} p$. Vamos entender como funciona essa identificação. Seja $U \subset \mathbb{P}_{\mathbb{K}} V$ aberto. Denotamos $U^{*} \subset V$ o aberto saturado de $U$ em $V$, isto é, $\pi^{-1} U$. Dados $f: U \rightarrow \mathbb{K}$ suave e $\varphi \in \operatorname{Lin}_{\mathbb{K}}(p, V / p)$ vetor tangente em $p$, seja $\hat{\varphi} \in \operatorname{Lin}_{\mathbb{K}}(p, V)$ um levantamento de $\varphi$. A derivação de $f$ por $\varphi$ em $p$ é dada por

$$
\varphi_{p} f=\left.\frac{d}{d t}\right|_{t=0} f \circ \pi([1+t \hat{\varphi}] p) .
$$

note que (como é necessário) a fórmula não depende do representante de $p$ e do levantamento $\hat{\varphi}$.

Aqui, e no que segue, frequentemente não faremos distinção na notação de um ponto em $\mathbb{P}_{\mathbb{K}} V$, de um representante deste em $V$ e do espaço 1-dimensional correspondente, quando o conceito ou expressão não depende da interpretação. Por exemplo, o subespaço $p^{\perp} \leq V$ é bem definido para todo $p \in \mathbb{P}_{\mathbb{K}} V$. 
Se $p$ é não-isotrópico, isto é, se $\langle p, p\rangle \neq 0$, podemos identificar naturalmente $V / p$ com $p^{\perp}$. Neste caso, nós interpretamos o espaço tangente como $\mathrm{T}_{p} \mathbb{P}_{\mathbb{K}} V=\operatorname{Lin}_{\mathbb{K}}\left(p, p^{\perp}\right)$. Este, por sua vez, herda a forma $\mathbb{R}$-bilinear

$$
\left(t_{1}, t_{2}\right):= \pm \frac{\operatorname{tr}_{\mathbb{R}}\left(t_{1}^{*} t_{2}\right)}{\operatorname{dim}_{\mathbb{R}} \mathbb{K}}
$$

onde $t_{1}, t_{2}: p \mapsto p^{\perp}$ são vetores tangentes, $t_{1}^{*}: p^{\perp} \rightarrow p$ é o mapa adjunto de $t_{1}$ com respeito a forma hermitiana, $\operatorname{tr}_{\mathbb{R}}\left(t_{1}^{*} t_{2}\right)$ denota o traço da aplicação $\mathbb{R}$-linear $t_{1}^{*} t_{2}: p \rightarrow p$ e o sinal pode ser escolhido de forma conveniente. Vamos nos referir a esta forma como a métrica da geometria clássica. No caso $\mathbb{K}=\mathbb{C}$, temos também a métrica hermitiana

$$
\left\langle t_{1}, t_{2}\right\rangle:= \pm \operatorname{tr}_{\mathbb{C}}\left(t_{1}^{*} t_{2}\right)
$$

É fácil ver que $\operatorname{Re}\left\langle t_{1}, t_{2}\right\rangle=\left(t_{1}, t_{2}\right)$. Obviamente, a métrica (hermitiana) depende suavemente de $p$ não-isotrópico. Se a forma hermitiana em $V$ é não-degenerada, então a métrica é não-degenerada.

A assinatura dos pontos dividem $\mathbb{P}_{\mathbb{K}} V$ em três partes: pontos negativos, pontos isotrópicos e pontos positivos, definidos respectivamente como

$$
\begin{aligned}
& \mathrm{B} V:=\left\{p \in \mathbb{P}_{\mathbb{K}} V \mid\langle p, p\rangle<0\right\} \\
& \mathrm{S} V:=\left\{p \in \mathbb{P}_{\mathbb{K}} V \mid\langle p, p\rangle=0\right\}, \\
& \mathrm{E} V:=\left\{p \in \mathbb{P}_{\mathbb{K}} V \mid\langle p, p\rangle>0\right\}
\end{aligned}
$$

Exemplo 1. Tome

(1) $\mathbb{K}=\mathbb{C}, \operatorname{dim}_{\mathbb{C}} V=2$, a forma com assinatura ++ e o sinal + na definição da métrica hermitiana. Obtemos a esfera 2-dimensional redonda.

(2) $\mathbb{K}=\mathbb{C}, \operatorname{dim}_{\mathbb{C}} V=2$, a forma com assinatura +- e o sinal - na definição da métrica hermitiana. Seja $p \in \mathbb{P}_{\mathbb{C}} V$ não-isotróprico. Pela decomposição ortogonal $V=p \oplus p^{\perp}$ segue que a métrica hermitiana em $T_{p} \mathbb{P}_{\mathbb{C}} V$ é positivo definida. Ganhamos dois discos de Poincaré hiperbólicos $\mathrm{B} V$ e $\mathrm{E} V$, colados pelo absoluto $\mathrm{S} V$.

(3) $\mathbb{K}=\mathbb{R}, \operatorname{dim}_{\mathbb{R}} V=3$, a forma com assinatura ++- e o sinal - . O disco B $V$ é o disco hiperbólico de Beltrami-Klein.

(4) $\mathbb{K}=\mathbb{C}, \operatorname{dim}_{\mathbb{C}} V=3$, a forma com assinatura ++- e o sinal -. A 4-bola aberta $\mathrm{B} V$ é o plano hiperbólico complexo $\mathbb{H}_{\mathbb{C}}^{2}$.

De maneira similar, podemos descrever muitas outras geometrias: Esféricas, FubiniStudy, hiperbólicas incluindo as de curvatura seccional constante e curvatura holomorfa constante, algumas geometrias lorentzianas como espaços de de Sitter e anti de Sitter. 


\subsubsection{Algumas construções geométricas.}

Exemplo 2. Tomemos $W \leq V, \operatorname{dim}_{\mathbb{R}} W=2$ tal que $W$ não é $\mathbb{C}$-espaço vetorial (caso contrário, $\mathbb{P}_{\mathbb{C}} W$ é simplesmente um ponto em $\mathbb{P}_{\mathbb{C}} V$ ). Suponhamos que a forma hermitiana, restrita a $W$, é real e não nula. É fácil entender que $\mathbb{K} W \simeq \mathbb{K} \otimes_{\mathbb{R}} W$. Então, o círculo

$$
\mathrm{G} W:=\mathbb{P}_{\mathbb{K}} W=\mathbb{P}_{\mathbb{R}} W \simeq \mathbb{S}^{1}
$$

é dito uma geodésica em $\mathbb{P}_{\mathbb{K}} V$. A linha projetiva $\mathbb{P}_{\mathbb{K}}(\mathbb{K} W)$ é dita a linha projetiva da geodésica. De fato, conforme provaremos na seção 1.3.1, tal construção é de fato uma geodésica no sentido usual com respeito a métrica nos pontos não isotrópicos, e toda geodésica em $\mathbb{P}_{\mathbb{K}} V$ tem essa mesma origem.

Exemplo 3. Seja $W \leq V, \operatorname{dim}_{\mathbb{R}} W=2$ nas condições de (2) do exemplo 1 . A parte real da forma hermitiana restrita a $W$ pode ser indefinida não-degenerada, definida, não-nula degenerada, ou nula. O círculo $\mathbb{P}_{\mathbb{K}} W$ é chamado respectivamente hiperciclo, círculo métrico, horociclo e absoluto. Dentro de cada um dos discos de Poincaré E $V$ e B $V$, estes são os usuais hiperciclos, círculos métricos e horociclos.

\subsubsection{Fatos preliminares.}

Seja $p \in \mathbb{P}_{\mathbb{K}} V$ não-isotrópico. Iremos utilizar a seguinte notação para a decomposição ortogonal:

$$
V=p \oplus p^{\perp}, \quad v=\pi^{\prime}[p]+\pi[p] v,
$$

onde

$$
\pi^{\prime}[p] v:=\frac{\langle p, v\rangle}{\langle p, p\rangle} p \in \mathbb{K} p \quad \text { e } \quad \pi[p] v:=v-\frac{\langle p, v\rangle}{\langle p, p\rangle} p \in p^{\perp}
$$

não dependem da escolha de representante para $p$.

A forma hermitiana restrita a um $\mathbb{K}$-subespaço bidimensional $W \leq V$ pode ser nula, definida, indefinida não-degenerada ou não-nula degenerada. A correspondente linha projetiva $\mathbb{P}_{\mathbb{K}} W$ será chamada respectivamente nula, esférica, hiperbólica ou euclidiana.

Lema 1. (1) Seja $L$ uma linha projetiva. Para todo $p \in L$ não-isotrópico existe um único $q \in L$ ortogonal a $p$, isso é, $\langle p, q\rangle=0$.

(2) Pontos isotrópicos numa linha projetiva hiperbólica formam uma $(n-1)$-esfera, onde $n=\operatorname{dim}_{\mathbb{R}} \mathbb{K}$. Linhas projetivas euclidianas possuem um único ponto isotrópico.

Quando $p \in V$ é não isotrópico, temos a identificação $\mathrm{T}_{p} \mathbb{P}_{\mathbb{K}} V=\operatorname{Lin}_{\mathbb{K}}\left(p, p^{\perp}\right)=p^{\perp}\langle p,-\rangle$. Em termos dessa caracterização, o mapa adjunto e o traço de um vetor tangente são facilmente calculado. 
Lema 2. (1) O mapa adjunto a $v\langle p,-\rangle$ é dado por $(v\langle p,-\rangle)^{*}=p\langle v,-\rangle$.

(2) Seja $p \in \mathbb{P}_{\mathbb{K}} V$ não-isotrópico e seja $v \in V$. Então o traço do mapa $\mathbb{R}$-linear $t:=v\langle p,-\rangle$ é dado por $\operatorname{tr}_{\mathbb{R}} t=\operatorname{dim}_{\mathbb{R}} \mathbb{K} \cdot \operatorname{Re}\langle p, v\rangle$

Definição 2. Seja $W \subset V \mathbb{R}$-subespaço vetorial. Dizemos que um ponto $p \in W$ é projetivamente suave em $W$ se $\operatorname{dim}_{\mathbb{K}}(p \mathbb{K} \cap W)=\min _{0 \neq w \in W} \operatorname{dim}_{\mathbb{R}}(\cap W)$.

Não é difícil provar que os pontos projetivamente suaves em $W$ formam uma região aberta em $\mathbb{P}_{\mathbb{K}} W$. Além disso, temos

Lema 3. [AGG, Lemma 4.2.2] Seja $W \subset V$ um $\mathbb{R}$-subespaço vetorial, seja $p \in W$ ponto projetivamente suave em $W$, e seja $\varphi \in \operatorname{Lin}_{\mathbb{K}}(p, V)$. Então $t_{\varphi} \in \mathrm{T}_{p} \mathbb{P}_{\mathbb{K}} W$ se e somente se $\varphi p \in W+\mathbb{K} p$

O vetor tangente de uma curva suave pode ser expressado em termos da identificação $\mathrm{T}_{p} \mathbb{P}_{\mathbb{K}} V=\operatorname{Lin}_{\mathbb{K}}\left(p, p^{\perp}\right)$ :

Lema 4. Seja $c:[a, b] \rightarrow \mathbb{P}_{\mathbb{K}} V$ uma curva suave e seja $c_{0}:[a, b] \rightarrow V$ um levantento suave de $c$ para $V$. Se o ponto $c\left(t_{0}\right)$ é não-isotrópico, então o vetor tangente $\dot{c}\left(t_{0}\right): c_{0}\left(t_{0}\right) \rightarrow c_{0}\left(t_{0}\right)^{\perp}$ é dado por $\dot{c}\left(t_{0}\right): c_{0}\left(t_{0}\right) \mapsto \pi\left[c\left(t_{0}\right)\right] \dot{c}_{0}\left(t_{0}\right)$

\subsection{Geodésicas}

Vamos relembrar a definição do exemplo 1 (1). Tome um $\mathbb{R}$-subespaço bidimensional $W \subset V$ tal que a forma hermitiana, restrita a $W$, é real e não nula. É imediato que $\mathbb{K} W \simeq \mathbb{K} \otimes_{\mathbb{R}} W$. Logo, $\mathbb{P}_{\mathbb{K}} W=\mathbb{P}_{\mathbb{R}} W$. O círculo $\mathrm{G} W:=\mathbb{P}_{\mathbb{K}} W$ é, por definição, uma geodésica. (O corolário X.X relaciona esta definição com a tradicional). A geodésica $W$ gera a linha projetiva $\mathbb{P}_{\mathbb{K}}(\mathbb{K} W)$. Uma geodésica é dita esférica, hiperbólica ou euclidiana a depender da natureza de sua linha projetiva. Começamos provando alguns fatos elementares sobre geodésicas:

Lema 5. (1) Sejam $g_{1}, g_{2} \in \mathbb{P}_{\mathbb{K}} V$ pontos distintos e não ortogonais. Então existe uma úniga geodésica contendo $g_{1}$ e $g_{2}$.

(2) Seja $p \in \mathbb{P}_{\mathbb{K}} V$ não-isotrópico e seja $0 \neq t \in \mathrm{T}_{p} \mathbb{P}_{\mathbb{K}} V, t: p \rightarrow p^{\perp}$. Então existe uma única geodésica com vetor tangente $t$ em $p$. Essa geodésica é dada pelo subespaço $W=\mathbb{R} p+$ $\mathbb{R} t p$.

Demonstração. (1) Seja $W=\mathbb{R} g_{1}+\mathbb{R}\left\langle g_{1}, g_{2}\right\rangle g_{2}$. É claro que $g_{1}, g_{2} \in \mathrm{G} W$. Se $g_{1}, g_{2} \in W^{\prime}$, então $W^{\prime}=\mathbb{R} k_{1} g_{1}+\mathbb{R} k_{2} g_{2}$ para certos $k_{1}, k_{2} \in \mathbb{K}$ tais que $k_{1} \bar{k}_{2}\left\langle g_{1}, g_{2}\right\rangle \in \mathbb{R}^{*}$ (pois $W^{\prime}$ é geodésica). Então, $W^{\prime}=\mathbb{R} k_{1} g_{1}+\mathbb{R} k_{1} \bar{k}_{2}\left\langle g_{1}, g_{2}\right\rangle k_{2} g_{2}=k_{1} W$, isso é, $\mathrm{G} W^{\prime}=\mathrm{G} W$.

(2) A geodésica $G W$, onde $W=\mathbb{R} p+\mathbb{R} t p$, não depende da escolha de $p \in \mathbb{K} p$. Pelo lema $3, t$ é vetor tangente a $\mathrm{G} W$ em $p$. Seja $\mathrm{G} W^{\prime}$ uma geodésica com vetor tangente $t$. Podemos 
escolher $W^{\prime}$ tal que $p \in W^{\prime}$. Pelo lema 3, $t p \in W^{\prime}+\mathbb{K} p$. Então, $t p \in p^{\perp}$ implica $t p \in W^{\prime}$. Ou seja, $W^{\prime}=\mathbb{R} p+\mathbb{R} t p$

Denotamos por $\mathrm{G}\left\{g_{1}, g_{2} \nmid\right.$ a geodésica que contem os pontos distintos e não ortogonais $g_{1}, g_{2} \in \mathbb{P}_{\mathbb{K}} V$.

Tomemos $g_{1}, g_{2} \in \mathbb{P}_{\mathbb{K}} V$ distintos e ortogonais. Assuma que a linha projetiva L gerada por $\mathrm{G} l g_{1}, g_{2}$ lé não nula. Ao menos um dos $g_{1}, g_{2}$ é não-isotrópico — digamos, $g_{1}$. Toda geodésica em $\mathrm{L}$ passando por $g_{1}$ tem a forma $\mathrm{G} W \operatorname{com} W=\mathbb{R} q+\mathbb{R} g_{1}$, onde $g_{1} \neq q \in \mathrm{L}, \mathrm{e}\left\langle q, g_{1}\right\rangle \in \mathbb{R}^{*}$. Então, $\pi\left[g_{1}\right] q \in \mathrm{G} W$.Pelo lema 1 (1), $g_{2}$ é o único ponto em L ortogonal a $g_{1}$. Portanto, $\pi\left[g_{1}\right] q=g_{2}$ em $\mathbb{P}_{\mathbb{K}} V$. Ou seja, toda geodésica em L que passa por $g_{1}$ necessariamente passa por $g_{2}$. Em particular, toda geodésica numa linha projetiva euclidiana passa por seu ponto isotrópico (veja lema 1 (2)). Neste caso, no mapa afim $\mathbb{K}$ de pontos não-isotrópicos de $\mathrm{L}$, as geodésicas correspondem a linhas retas. Isto justifica o termo 'euclidiana'. Como a métrica é na realidade nula nas linhas projetivas euclidianas, talvez o termo mais apropriado fosse linha afim.

\subsection{Conexão de Levi-Civita}

No que segue, assumimos que a forma hermitiana $\langle-,-\rangle$ é não-degenerada. Em particular, B $V$ e E $V$ são munidos de métrica pseudo-riemanniana. Além disso, usaremos as seguintes convenções:

Seja $p \in \mathbb{P}_{\mathbb{K}} V$ não-isotrópico. Estendendo por zeros, podemos considerar qualquer vetor tangente $t: p \rightarrow p^{\perp}$ como uma aplicação linear $t \in \operatorname{Lin}_{\mathbb{K}}(V, V)$. Dessa forma, $\mathrm{T}_{p} \mathbb{P}_{\mathbb{K}} V=$ $\operatorname{Lin}_{\mathbb{K}}\left(p, p^{\perp}\right) \subset \operatorname{Lin}_{\mathbb{K}}(V, V)$. É fácil entender que valem $t=t \pi^{\prime}[p], t=\pi[p] t, t \pi[p]=\pi^{\prime}[p] t=0$, e $s t=0$ para todos vetores tangentes $s, t \in \operatorname{Lin}_{\mathbb{K}}(V, V)$ em $p$. Podemos fazer o mesmo no caminho inverso; Dado $t \in \operatorname{Lin}_{\mathbb{K}}(V, V)$ arbitrário, definimos o vetor tangente

$$
t_{p}:=\pi[p] t \pi^{\prime}[p]
$$

em $p$ (neste caso dizemos que o vetor tangente $t$ é observado em $p$ ).

Seja $U \subset V$ um aberto saturado (i.e., $\mathbb{K}^{*} U \subset U$ ) sem pontos isotrópicos. Um campo levantado sobre $U$ é uma aplicação suave $X: U \rightarrow \operatorname{Lin}_{\mathbb{K}}(V, V)$ tal que $X(p)_{p}=X(p)$ and $X(k p)=X(p)$ para todo $p \in U$ e $k \in \mathbb{K}^{*}$. Em outras palavras, $X$ consistentemente define um um campo suave sobre $\mathbb{P}_{\mathbb{K}} U$.

Definição 3. Toda aplicação $t \in \operatorname{Lin}_{\mathbb{K}}(V, V)$ dá origem a seu campo (levantado) $T$ espalhado: Este é dado pela regra $T(p)=t_{p}$, que é definido para todo $p$ não-isotrópico.

Para $t \in \operatorname{Lin}_{\mathbb{K}}(V, V)$ e $X$ campo levantado, definimos

$$
\nabla_{t} X(p):=\left(\left.\frac{d}{d \varepsilon}\right|_{\varepsilon=0} X((1+\varepsilon t) p)\right)_{p}
$$


Como $\pi[k p]=\pi[p]$ e $\pi^{\prime}[k p]=\pi^{\prime}[p]$ para todo $p \in U$ e $k \in \mathbb{K}^{*}$, o campo $p \mapsto \nabla_{Y(p)} X$ é um campo levantado para arbitrários $X, Y$ campos levantados sobre $U$. Segue da definição que $\nabla$ é uma conexão afim.

Lema 6. Seja $p \in \mathbb{P}_{\mathbb{K}} V$ não-isotrópico e seja $t$ vetor tangente em $p$. Então vale

$$
\left.\frac{d}{d \varepsilon}\right|_{\varepsilon=0} \pi^{\prime}[p+\varepsilon t p]=-\left.\frac{d}{d \varepsilon}\right|_{\varepsilon=0} \pi[p+\varepsilon t p]=t+t^{*}
$$

Demonstração. Pela definição de $\pi^{\prime}$, temos $\pi^{\prime}[p+\varepsilon t p]=\frac{\langle p+\varepsilon t p,-\rangle}{\langle p, p\rangle+\varepsilon^{2}\langle t p, t p\rangle}(p+\varepsilon t p)$. Derivando a fórmula, temos

$$
\begin{gathered}
\left.\frac{d}{d \varepsilon}\right|_{\varepsilon=0} \frac{\langle p+\varepsilon t p,-\rangle}{\langle p, p\rangle+\varepsilon^{2}\langle t p, t p\rangle}(p+\varepsilon t p)=\frac{\langle t p,-\rangle}{\langle p, p\rangle} p+\frac{\langle p,-\rangle}{\langle p, p\rangle} t p . \\
\text { O segundo termo é } t \pi^{\prime}[p]=t \text {. Denotando } \varphi:=\frac{\langle t p,-\rangle}{\langle p, p\rangle} p, \text { temos } \\
\langle t x, y\rangle=\left\langle t \pi^{\prime}[p] x, y\right\rangle=\left\langle\frac{\langle p, x\rangle}{\langle p, p\rangle} t p, y\right\rangle=\frac{\langle x, p\rangle}{\langle p, p\rangle}\langle t p, y\rangle=\left\langle x, \frac{\langle t p, y\rangle}{\langle p, p\rangle} p\right\rangle=\langle x, \varphi y\rangle
\end{gathered}
$$

para todos $x, y \in V . \operatorname{Logo}, t^{*}=\varphi$

Lema 7. Seja $p \in \mathbb{P}_{\mathbb{K}} V$ não-isotrópico. Sejam $s$ e $t$ vetores tangentes em $p$. Então

$$
\nabla_{T} S(x)=\left(s \pi[x] t-t \pi^{\prime}[x] s\right)_{x}
$$

para todo $x \in \mathbb{P}_{\mathbb{K}} V$ não-isotrópico, onde os campos $S$ e $T$ são respectivamente campos espalhados de $s$ e $t$. Em particular, $\nabla_{T} S(p)=0$.

Demonstração. Pelo lema anterior,

$$
\begin{aligned}
\nabla_{T} S(x) & =\nabla_{t_{x}} S(x)=\left(\left.\frac{d}{d \varepsilon}\right|_{\varepsilon=0} S\left(x+\varepsilon t_{x} x\right)\right)_{x}=\left(\left.\frac{d}{d \varepsilon}\right|_{\varepsilon=0} \pi\left[x+\varepsilon t_{x} x\right] s \pi^{\prime}\left[x+\varepsilon t_{x} x\right]\right)_{x}= \\
& =\left(-\left(t_{x}+\left(t_{x}\right)^{*}\right) s \pi^{\prime}[x]+\pi[x] s\left(t_{x}+\left(t_{x}\right)^{*}\right)\right)_{x}=\left(s \pi[x] t-t \pi^{\prime}[x] s\right)_{x}
\end{aligned}
$$

já que $\pi[x]\left(t_{x}\right)^{*}=\left(t_{x}\right)^{*} \pi^{\prime}[x]=0$

Lema 8. $\nabla$ é a conexão de Levi-Civita da métrica (hermitiana) de toda componente de $\mathbb{P}_{\mathbb{K}} V \backslash S V$.

Demonstração. Seja $p \in \mathbb{P}_{\mathbb{K}} V$ não-isotrópico. Sejam $S$ e $T$ campos levantados locais com $S(p):=s$ e $T(p):=t$. Para mostrar que $\left(\nabla_{S} T-\nabla_{T} S-[S, T]\right)(p)=0$, lembramos que a torção é um tensor e que portanto podemos assumir que $S$ e $T$ são espalhados respectivamente por $S$ e $t$ (veja Definição 3). Segue do lema 7 que $\nabla_{S} T(p)=\nabla_{T} S(p)=0$. O fato que $[S, T](p)=0$ segue assim: Seja $f$ função suave e seja $\hat{f}$ seu levantamento para $V$. Por definição, $T(x) f=$ $\left.\frac{d}{d \varepsilon}\right|_{\varepsilon=0} \hat{f}(x+\varepsilon \pi[x] t x)$. Logo 


$$
\begin{gathered}
S(p)(T f)=\left.\frac{d}{d \delta}\right|_{\delta=0}\left(\left.\frac{d}{d \varepsilon}\right|_{\varepsilon=0} \hat{f}(p+\delta s p+\varepsilon \pi[p+\delta s p] t(p+\delta s p))\right)= \\
=\left.\frac{d}{d \delta}\right|_{\delta=0}\left(\left.\frac{d}{d \varepsilon}\right|_{\varepsilon=0} \hat{f}(p+\delta s p+\varepsilon \pi[p+\delta s p] t p)\right)= \\
=\left.\frac{d}{d \delta}\right|_{\delta=0}\left(\left.\frac{d}{d \varepsilon}\right|_{\varepsilon=0} \hat{f}\left(p+\delta s p+\varepsilon t p-(p+\delta s p) \frac{k_{0} \varepsilon \delta}{1+\delta^{2}\langle s p, s p\rangle /\langle p, p\rangle}\right)\right),
\end{gathered}
$$

onde denotamos $k_{0}:=\langle s p, t p\rangle /\langle p, p\rangle$. Como $\hat{f}(p k)=\hat{f}(p)$ para todo $k \in \mathbb{K}^{*}$, segue que

$$
\hat{f}\left(\left(1-k_{0} \varepsilon \delta\right) p+\delta\left(1-k_{0} \varepsilon \delta\right) s p+\varepsilon t p\right)=\hat{f}\left(p+\delta s p+\frac{\varepsilon}{1-k_{0} \varepsilon \delta} t p\right) .
$$

Sendo $f$ suave,

$$
\begin{gathered}
S(p)(T f)=\left.\frac{d}{d \delta}\right|_{\delta=0}\left(\left.\frac{d}{d \varepsilon}\right|_{\varepsilon=0} \hat{f}\left(p+\delta s p+\varepsilon t p-k_{0} \varepsilon \delta(p+\delta s p)\right)\right)= \\
=\left.\frac{d}{d \delta}\right|_{\delta=0}\left(\left.\frac{d}{d \varepsilon}\right|_{\varepsilon=0} \hat{f}\left(p+\delta s p+\frac{\varepsilon}{1-k_{0} \varepsilon \delta} t p\right)\right)=\left.\frac{d}{d \delta}\right|_{\delta=0}\left(\left.\frac{d}{d \varepsilon}\right|_{\varepsilon=0} \hat{f}(p+\delta s p+\varepsilon t p)\right) .
\end{gathered}
$$

Logo, $S(p)(T f)=T(p)(S f)$, isto é, $[S, T](p)=0$.

Para verificar que $v(S, T)(p)=\left(\nabla_{\nu} S(p), T(p)\right)+\left(S(p), \nabla_{v} T(p)\right)$ para um vetor tangente $v$ em $p$, definimos $\varphi_{1}:=\left.\frac{d}{d \varepsilon}\right|_{\varepsilon=0} S(p+\varepsilon v p)$ e $\varphi_{2}:=\left.\frac{d}{d \varepsilon}\right|_{\varepsilon=0} T(p+\varepsilon v p)$. Então,

$$
\begin{aligned}
& \pm \operatorname{dim}_{\mathbb{R}} \mathbb{K}\left(\nabla_{v} S(p), T(p)\right)= \pm \operatorname{dim}_{\mathbb{R}} \mathbb{K}\left(\pi[p] \varphi_{1} \pi^{\prime}[p], T(p)\right)=\operatorname{tr}_{\mathbb{R}}\left(\left(\pi[p] \varphi_{1} \pi^{\prime}[p]\right)^{*} T(p)\right)=\operatorname{tr}_{\mathbb{R}}\left(\varphi_{1}^{*} T(p)\right), \\
& \pm \operatorname{dim}_{\mathbb{R}} \mathbb{K}\left(S(p), \nabla_{v} T(p)\right)=\operatorname{tr}_{\mathbb{R}}\left(S^{*}(p) \varphi_{2}\right), \mathrm{e} \\
& \quad \pm \operatorname{dim}_{\mathbb{R}} \mathbb{K} v(S, T)(p)=\left.\frac{d}{d \varepsilon}\right|_{\varepsilon=0} \operatorname{tr}_{\mathbb{R}}\left(S^{*}(p+\varepsilon v p) T(p+\varepsilon v p)\right)=\operatorname{tr}_{\mathbb{R}}\left(\varphi_{1}^{*} T(p)\right)+\operatorname{tr}_{\mathbb{R}}\left(S^{*}(p) \varphi_{2}\right) .
\end{aligned}
$$

Por um argumento similar se prova o caso hermitiano.

\subsubsection{Transporte paralelo ao longo de geodésicas}

Definição 4. Sejam $p, q \in \mathbb{P}_{\mathbb{K}} V$. Definimos como a tância entre $p$ e $q$ a expressão:

$$
\operatorname{ta}(p, q):=\frac{\langle p, q\rangle\langle q, p\rangle}{\langle p, p\rangle\langle q, q\rangle}
$$

Note que essa expressão não depende de representantes de $p$ e $q$.

A tância recebe esse nome por ser intimamente relacionada com a distância nas geometrias clássicas ${ }^{1}$

1 De fato, a distância é uma função monótona da tância. Por ser algébrica, envolvendo apenas a forma hermitiana, é frequentemente útil utilizá-la em detrimento da própria distância, quando o contexto permite. Essa abordagem será utilizada no próximo capítulo 
Definição 5. Seja $p \in \mathbb{P}_{\mathbb{K}} V$ não-isotrópico, seja $t$ vetor tangente em $p$, e seja $T$ o campo espalhado por $t$. O campo levantado suave

$$
\operatorname{Tn}(t)(-):=\frac{\mathrm{T}(-)}{\operatorname{ta}(p,-)}
$$

é definido para todo ponto fora de $\mathbb{P}_{\mathbb{K}} p^{\perp} \cup \mathrm{S} V$.

Lema 9. Seja G uma geodésica e seja $t$ um vetor tangente não nulo de $\mathrm{G}$ num ponto nãoisotrópico $p \in \mathrm{G}$. Então o campo $\operatorname{Tn}(t)$ é não nulo e tangente a $\mathrm{G}$ em todo ponto em que $\operatorname{Tn}(t)$ é definido.

Demonstração. Seja $g \in \mathrm{G}$ não-isotrópico e não ortogonal a $p$. Claramente $\varphi:=\operatorname{Tn}(t)(g) \neq 0$, já que $\pi[g] t \pi^{\prime}[g]=0$ implicaria $g \in p^{\perp}$. Pelo lema 5 (2), $\mathrm{G}=\mathrm{G} W$ com $W=\mathbb{R} p+\mathbb{R} t p$. Podemos assumir que $g \in W$. Logo, $\varphi g \in W$ e $\operatorname{Tn}(t)(g)$ é tangente a $\mathrm{G}$ em $g$ pelo lema 3 .

Lema 10. Sejam $p, q \in \mathbb{P}_{\mathbb{K}} V$ distintos e não-ortogonais, com $p$ não-isotrópico. Denotamos por $\mathrm{G}[p, q]$ o segmento orientado ${ }^{2}$ da geodésica $\mathrm{G}\langle p, q$ q que não contém o ponto ortogonal a $p$. Seja $\varphi: V \rightarrow V$ dada por $\varphi=q\langle p, q\rangle^{-1}\langle p,-\rangle$. Então $\varphi_{p}$ é tangente ao segmento orientado $\mathrm{G}[p, q]$ em $p$.

Demonstração. $\mathrm{O}$ vetor tangente $\varphi_{p}$ não depende da escolha de representantes para $p$ e $q$. Podemos assumir que $\langle p, p\rangle=\sigma$ e $\langle p, q\rangle=\sigma a$, onde $\sigma \in\{-1,+1\}$ e $a>0$. Claramente, $\varphi_{p}$ : $p \mapsto(1 / a) \pi[p] q(1 / a)$. A curva $c_{0}(t):=(1-t) p+t q, t \in[0,1]$, parametriza um levantamento de $\mathrm{G}[p, q]$. De fato, $\langle p,(1-t) p+t q\rangle=0$ siginifca que $(1-a) t=1$, o que é impossível. Pelo lema 4 , a aplicação linear $\dot{c}(0): p \mapsto \pi[p] q$ é tangente a $\mathrm{G}[p, q]$ em $p$.

Lema 11. Seja $p \in \mathbb{P}_{\mathbb{K}} V$ não-isotrópico, seja $t$ um vetor tangente em $p$ e seja $T$ o campo espalhado de $t$. Então, para todo $x$ não-isotrópico, vale

$$
T(x)(\operatorname{ta}(p,-))=-2 \operatorname{ta}(p, x) \operatorname{Re} \frac{\langle t x, x\rangle}{\langle x, x\rangle}
$$

Demonstração. A prova é um cálculo direto:

$$
\begin{aligned}
T(x)(\operatorname{ta}(p,-)) & =\left.\frac{d}{d \varepsilon}\right|_{\varepsilon=0} \frac{\langle p, x+\varepsilon \pi[x] t x\rangle\langle x+\varepsilon \pi[x] t x, p\rangle}{\langle p, p\rangle\left(\langle x, x\rangle+\varepsilon^{2}\langle\pi[x] t x, \pi[x] t x\rangle\right)}=\frac{\langle p, \pi[x] t x\rangle\langle x, p\rangle+\langle p, x\rangle\langle\pi[x] t x, p\rangle}{\langle p, p\rangle\langle x, x\rangle}= \\
& =-\frac{\langle p, x\rangle\langle x, t x\rangle\langle x, p\rangle+\langle p, x\rangle\langle t x, x\rangle\langle x, p\rangle}{\langle p, p\rangle\langle x, x\rangle^{2}}=-2 \operatorname{ta}(p, x) \operatorname{Re} \frac{\langle t x, x\rangle}{\langle x, x\rangle}
\end{aligned}
$$

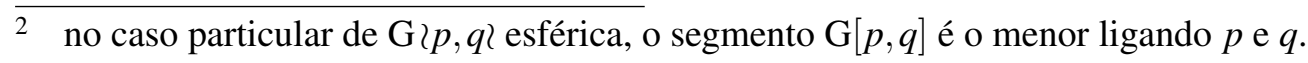


Lema 12. Seja $\mathrm{G}$ uma geodésica, seja $t$ um vetor tangente não nulo a $\mathrm{G}$ num ponto não isotrópico $p \in \mathrm{G}$, e seja $h \in \mathrm{T}_{p} \mathrm{~L}$, onde $\mathrm{L}$ é a linha projetiva de $G$. Então, para todo ponto $g \in \mathrm{G}$ não-isotrópico não ortogonal a $p$, vale

$$
\nabla_{\operatorname{Tn}(t)(g)} \operatorname{Tn}(h)=0
$$

Demonstração. Denote por $H$ e $T$ os campos respectivamente espalhados por $h$ e $t$. É suficiente mostrar que $\left(\nabla_{T(g)} \frac{H(-)}{\operatorname{ta}(p,-)}\right) g=0$. Pelo lema $5(2), \mathrm{G}=\mathrm{G} W$ com $W=\mathbb{R} p+\mathbb{R} t p$. Podemos tomar $W$ tal que $g \in W$. Pelos lemas 6 e 11 ,

$$
\begin{gathered}
\left(\nabla_{T(g)} \frac{H(-)}{\operatorname{ta}(p,-)}\right) g=T(g)\left(\frac{1}{\operatorname{ta}(p,-)}\right) H(g) g+\frac{1}{\operatorname{ta}(p, g)}\left(\nabla_{T(g)} H\right) g= \\
=\frac{1}{\operatorname{ta}(p, g)} \pi[g]\left(2 \frac{\langle t g, g\rangle}{\langle g, g\rangle} h g+h \pi[g] t g-t \pi^{\prime}[g] h g\right) .
\end{gathered}
$$

Segue do lema 3 que $h p=k t p$ para algum $k \in \mathbb{K}$ já que ambos $h$ e $t$ são tangentes a L em $p$. De $h p^{\perp}=t p^{\perp}=0$, concluímos que $h g=k t g$. Por fim, de $\pi[g]=1-\pi^{\prime}[g], h t g=0,\langle t g, g\rangle \in \mathbb{R}$, e $h g=k t g$, obtemos

$$
h \pi[g] \operatorname{tg}=-h \pi^{\prime}[g] \operatorname{tg}=-h g \frac{\langle g, t g\rangle}{\langle g, g\rangle}=-\frac{\langle t g, g\rangle}{\langle g, g\rangle} h g
$$

e

$$
t \pi^{\prime}[g] h g=k t \pi^{\prime}[g] t g=k t g \frac{\langle g, t g\rangle}{\langle g, g\rangle}=\frac{\langle t g, g\rangle}{\langle g, g\rangle} h g
$$

Os lemas 12,9 e 5 (2) tem como consequência o seguinte

Corolário 1. Fora dos pontos isotrópicos, uma geodésica no sentido do exemplo 2 é uma geodésica no sentido da conexão de Levi-Civita $\nabla$. Toda geodésica dessa conexão têm essa natureza. 



\section{ESFÉRICAS HIPERBÓLICAS COM PONTOS CÔNICOS}

\subsection{Introdução}

No trabalho germinal [Thu], W. P. Thurston estudou o espaço de 2-esferas planas com pontos cônicos de curvatura prescrita. Tais espaços possuem estrutura de variedade hiperbólica complexa, mas em geral não são completos. Seu completamento tem uma estrutura natural de variedade cônica complexa hiperbólica que é orbifold exatamente quando as curvaturas prescritas satisfazem uma simples condição. Dessa forma, Thurston recriou os célebres 7 quocientes compactos não-aritméticos da 2-bola holomorfa (abreviando, quocientes não-aritméticos) que foram construídos por Deligne-Mostow [DM1], [DM2] a 30-35 anos atrás. Já que não queremos distinguir um quociente $Q$ de um recobrimento (não-ramificado) finito de $Q$, assumimos $Q$ variedade. Recentemente, 4 novos quocientes não-aritméticos foram construídos em [DPP]. Estes 4 exemplos são muito similares aos outros 7 . Cada um dos 11 contêm ao menos quatro curvas $\mathbb{C}$-fuchsianas, i.e., curvas suaves compactas holomorfas totalmente geodésicas.

Por sua vez, os quocientes aritméticos são conhecidos por serem de 2 tipos [McR]: O primeiro tipo e o segundo tipo que é relacionado à álgebras com divisão. Levando em conta a rigidez (Mostow-Prasad) dos quocientes, o número de quocientes é contável para cada tipo. Os tipos podem ser distinguidos pela presença de uma curva $\mathbb{C}$-fuchsiana: um quociente do primeiro tipo possui uma tal curva, enquanto um do segundo tipo não. Usando curvas $\mathbb{C}$-fuchsianas, definimos o tipo de um quociente arbitrário pelo mesmo critério e chegamos às seguintes questões.

Problema 1. Existe algum quociente não-aritmético do segundo tipo? Existem infinitos quocientes não-aritméticos do primeiro/segundo tipo? 
Esboço Neste artigo, nós desenvolvemos algumas ferramentas que permitem o estudo do espaço $C\left(a_{0}, a_{1}, \ldots, a_{n}\right)$ de 2-esferas com pontos cônicos de curvaturas prescritas $2 a_{0}, 2 a_{1}, \ldots, 2 a_{n}$, onde o conjunto dos demais pontos é dotado de geometria esférica de curvatura $\sigma:=1$ ou hiperbólica de curvatura $\sigma:=-1$. Com sorte, $C\left(a_{0}, a_{1}, \ldots, a_{n}\right)$ ou espaços relacionados podem jogar alguma luz no problema enunciado.

Exemplo 1 (Toy example.). De modo a esclarecer o panorama geral, nós primeiro olhamos para o caso mais simples de 4 pontos cônicos de fixadas curvaturas $2 a, \pi, \pi, \pi$, onde $a \in] 0, \frac{\pi}{2}$ [ quando $\sigma=1$ e $a \in] \frac{\pi}{2}, \pi[$ quando $\sigma=-1$. Nessa situação em particular, cada 2-esfera hiperbólica ou esférica pode ser cortada num triângulo sólido (disco fechado) limitado por um triângulo e morando no espaço modelo, i.e., na esfera redonda ou no plano hiperbólico. De fato, basta ligar o ponto cônico $p$ de curvatura $2 a$ com os outros 3 pontos cônicos por segmentos geodésicos simples tais que estes se interceptem apenas em $p$ (por exemplo, tomando os segmentos mais curtos) e então cortar a 2-sphere ao longo destes segmentos. $\mathrm{O}$ ponto $p$ se divide nos vértices do triângulo e os outros 3 pontos cônicos serão pontos médios dos lados do triângulo. Se dividirmos o triângulo sólido ao longo de uma mediana e então colar as correspondentes metades, nós modificamos o triângulo original com respeito a essa mediana. Ao fazê-lo, as 2-esferas correspondentes aos triângulos $t_{1}$ and $t_{2}$ são isométricas (orientação preservada) se e somente se $t_{1}$ e $t_{2}$ diferem por um número finito de tais modificações. Isso nos permite descrever o espaço $C\left(a, \frac{\pi}{2}, \frac{\pi}{2}, \frac{\pi}{2}\right)$ como o quociente de um disco topológico $S$, o espaço de triângulos orientados no sentido anti-horário de área fixada $\sigma(\pi-2 a)$, pelo grupo $M_{3}$ gerado por 3 involuções que são nada mais que modificações agindo em $S$. Em certo sentido, essa construção relembra a de [WaW].

\subsubsection{Caso geral}

Denotamos por $G$ o grupo de Lie de isometrias preservando orientação (=holomorfas) do espaço modelo. Então, $G:=\operatorname{PU}(2)$ quando $\sigma=1$ e $G:=\operatorname{PU}(1,1)$ quando $\sigma=-1$. Sejam $c_{0}, c_{1}, \ldots, c_{n}$ pontos cônicos de alguma 2 -sphere $\Sigma \in C\left(a_{0}, a_{1}, \ldots, a_{n}\right)$. Ganhamos uma representação de holonomia $\rho: F_{n} \rightarrow G$, onde $F_{n}:=\pi_{1}\left(\Sigma \backslash\left\{c_{0}, c_{1}, \ldots, c_{n}\right\}\right)$ é o grupo livre de posto $n$. Desse modo, obtemos um mapa de holonomia bem definido $h: C\left(a_{0}, a_{1}, \ldots, a_{n}\right) \rightarrow \operatorname{Rep}\left(F_{n}, G\right) / G$, $\Sigma \mapsto[\rho]$, para o espaço de representações $\operatorname{Rep}\left(F_{n}, G\right)$ considerado módulo conjugação por $G$.

Como no exemplo 1 , podemos ligar $c_{0} \operatorname{com} c_{1}, \ldots, c_{n}$ por $n$ segmentos geodésicos simples que se interceptam apenas em $c_{0}$ e então cortar $\Sigma$ ao longo de tais segmentos, ganhando um $2 n$-gono $P$ que limita um disco topológico fechado não necessariamente mergulhável no espaço modelo. Nomeando os vértices ímpares de $P$ que se originam de $c_{1}, \ldots, c_{n}$ com os mesmos símbolos e os pares, que se originam de $c_{0}$, de $p_{1}, \ldots, p_{n}$, vemos que o ângulos internos de $P$ nos $p_{j}$ 's somam $2 \pi-2 a_{0}$, que os ângulos interiores de $P$ em $c_{j}$ serão $2 \pi-2 a_{j}$, e que os lados de $P$ adjacentes à $c_{j}$ têm mesmo comprimento para todo $j=1, \ldots, n$. De certa maneira, $P$ limita um domínio fundamental para $\Sigma$. 
Uma escolha de tais segmentos distingue geradores $r_{0}, r_{1}, \ldots, r_{n}$ de $F_{n}$ tal que $F_{n}=$ $\left\langle r_{0}, r_{1}, \ldots, r_{n} \mid r_{n} \ldots r_{1} r_{0}=1\right\rangle$. Um apropriado grupo de tranças $\bar{M}_{n}$ (veja Definição 2) age em $F_{n}$ e preserva o conjunto de classes de conjugação dos $r_{j}$ 's. Uma outra escolha destes segmentos é dada no nível de representações por um elemento $m \in \bar{M}_{n}$ que transforma $[\rho]$ em $[\rho \circ m]$. (Em geral, tais elementos $m$ não formam um subgrupo.) Portanto, a imagem do mapa de holonomia $h$ vive em uma espécie de variedade relativa de caracteres $R\left(a_{0}, a_{1}, \ldots, a_{n}\right) \subset \operatorname{Rep}\left(F_{n}, G\right) / G$ formada por todas as representações $[\rho]$ tais que as classes de conjugação de $\rho r_{0}, \rho r_{1}, \ldots, \rho r_{n}$ são as rotações em sentido anti-horário no espaço modelo pelos ângulos $2 a_{0}, 2 a_{1}, \ldots, 2 a_{n}$, listados talvez em ordem diferente. Noutras palavras, $R\left(a_{0}, a_{1}, \ldots, a_{n}\right)$ é o espaço de relações entre rotações de classe de conjugação prescrita (a ordem das classes não é fixada).

\subsubsection{2n-gonos hiperbólicos convexos}

Vamos olhar mais carinhosamente para o caso $\sigma=-1 \operatorname{com} a_{0}, a_{1}, \ldots, a_{n} \in\left[\frac{\pi}{2}, \pi[\right.$ tais que $\sum_{j} a_{j}>2 \pi$. Aqui, qualquer $2 n$-gono $P$ é convexo, i.e., todos seus ângulos interiores estão em $] 0, \pi]$, e portanto, o disco fechado correspondente é mergulhável no espaço modelo. Nesse caso, o espaço $C\left(a_{0}, a_{1}, \ldots, a_{n}\right)$ de 2-esferas hiperbólicas com pontos cônicos de curvatura $2 a_{0}, 2 a_{1}, \ldots, 2 a_{n}$ pode ser descrito como o quociente de uma componente de $R\left(a_{0}, a_{1}, \ldots, a_{n}\right)$ pela ação de $\bar{M}_{n}$ (veja Seção 2.5 e Teorema 2; veja também Seção 2.1.5).

\subsubsection{Geometria em $C\left(a_{0}, a_{1}, \ldots, a_{n}\right)$ e outros espaços relacionados}

Como esperamos munir $C\left(a_{0}, a_{1}, \ldots, a_{n}\right)$ com uma estrutura de orbifold hiperbólico complexo, é natural conjecturar que tal geometria descende de uma componente $C$ de $R\left(a_{0}, a_{1}, \ldots, a_{n}\right)$ e que $\bar{M}_{n}$ age discretamente em $C$ por isometrias. (Existe uma maneira mais astuta de construir espaços com geometria de forma similar, mas limitaremos inicialmente para essa variante mais simples.)

Na verdade, mesmo no primeiro caso considerado por Thurston, pode não existir nenhuma geometria hiperbólica em $C$. (No caso plano, podemos sempre escolher a estrutura advinda da área orientada; esta providencia uma estrutura complexa hiperbólica.) A escolha de uma 'boa' estrutura hiperbólica em $C$ é o principal desafio do projeto, e o primeiro passo é descrever as componentes de $R\left(a_{0}, a_{1}, \ldots, a_{n}\right)$ onde $\bar{M}_{n}$ age discretamente.

Podemos estabelecer a inexistência de geometria de curvatura constante numa componente de $R\left(a_{0}, a_{1}, a_{2}, a_{3}\right)$, compatível com a ação de $\bar{M}_{3}$, se essa ação é não discreta.

\subsubsection{Breve esboço da exposição}

Toda representação $[\rho] \in R\left(a_{0}, a_{1}, \ldots, a_{n}\right)$ pode ser interpretada como um $2 n$-gon marcado, um caminho geodésico por partes fechado e orientado, cujos vértices consecutivos $c_{1}, p_{1}, \ldots, c_{n}, p_{n}:=c_{0}$ são dados por $p_{j}:=R_{j} p_{j-1}$ para todo $1 \leqslant j \leqslant n$ (índices módulo $n$ ), 
onde $c_{j}$ é o ponto fixo da rotação $R_{j}:=\rho r_{j}, 0 \leqslant j \leqslant n$, e, sem perda de generalidade, $R_{0}$ é uma rotação anti-horária de ângulo $2 a_{0}$.

É conveniente lidar com os $2 n$-gonos com uma marcação esquecida $c_{0}$. Em outras palavras, consideramos o quociente $S\left(a_{0}, a_{1}, \ldots, a_{n}\right)$ de $R\left(a_{0}, a_{1}, \ldots, a_{n}\right)$ por um adequado grupo cíclico de ordem $n$ em $\bar{M}_{n}$. Assim, nós podemos colocar o ponto fixo da rotação $R_{0}$ em qualquer vértice $p_{j}$. Em termos de relações, o gerador do grupo cíclico transforma a relação $R_{n} \ldots R_{1} R_{0}=1$ na relação $R_{1} R_{n} \ldots\left(R_{1} R_{0} R_{1}^{-1}\right)=1$. Um certo grupo de tranças $M_{n}$ (veja Definição 3 ) gerado por modificações simples (cirurgias) nos $2 n$-gonos age em $S\left(a_{0}, a_{1}, \ldots, a_{n}\right)$ de tal forma que o mapa $R\left(a_{0}, a_{1}, \ldots, a_{n}\right) \rightarrow S\left(a_{0}, a_{1}, \ldots, a_{n}\right)$ induz uma bijeção entre as $\bar{M}_{n}$-orbitas e as $M_{n}$-orbitas. Obviamente, a discretude de $\bar{M}_{n}$ é equivalente a a discretude de $M_{n}$.

O próximo passo é descrever as componentes de $S\left(a_{0}, a_{1}, \ldots, a_{n}\right)$. A ordem cíclica de $a_{1}, \ldots, a_{n}$ em $P$ define o tipo de um $2 n$-gono $P$ e $M_{n}$ age transitivamente nos tipos. Portanto, precisamos descrever as componentes de um dado tipo e encontrar aquelas componentes onde $M$, o subgrupo de índice $(n-1)$ ! de elementos que preservam o tipo de $M_{n}$, age discretamente.

Procedemos por indução em $n$. Sem perda de generalidade, assumimos que $R_{0}$ e $R_{n}$ são rotações no sentido anti-horário por $2 a_{0}$ e $2 a_{n}$. A classe de conjugação de $R_{0} R_{n}$ é dada exatamente pela distância $d_{n}$ entre $c_{n}$ e $p_{n}$. Portanto, por indução, nós sabemos a topologia das fibras da aplicação $d_{n}: S\left(a_{0}, a_{1}, \ldots, a_{n}\right) \rightarrow\left[0, \infty\left[\right.\right.$. De fato, se $R_{0} R_{n}$ é uma rotação em sentido anti-horário por $2 a, a \in] 0, \pi$, então a fibra em questão é $S\left(a, a_{1}, \ldots, a_{n-1}\right) \times C_{R_{0} R_{n}}$, onde $C_{R_{0} R_{n}}$ é o centralizador de $R_{0} R_{n}$ in $G$, topologicamente um círculo. Se $R_{0} R_{n}=1$ ( $\operatorname{logo}, d_{n}=0$ ), então a fibra é $S\left(a_{1}, \ldots, a_{n-1}\right)$. Se $R_{0} R_{n}$ é uma isometria parabólica ou hiperbólica, então a fibra é $S\left(a^{*}, a_{1}, \ldots, a_{n-1}\right) \times C_{R_{0} R_{n}}$, onde $a^{*}$ é a classe de conjugação de $R_{0} R_{n}$ e $C_{R_{0} R_{n}}$ é topologicamente uma linha. (Isso significa que, para a indução, precisamos considerar as relações entre isometrias de classes de conjugação prescritas, não necessariamente elípticas. Nós não o fazemos nesse artigo em particular porque o que estudamos aqui é principalmente o caso de $n=3$ e $\sigma=-1$.) Coletando informações sobre a topologia das fibras de $d_{n}$, incluíndo aquelas sobre pontos singulares de $d_{n}$, nós chegamos a descrição das componentes e suas respectivas topologias (veja Proposição 1).

Também, no caso $n=3$ e $\sigma=-1$, enxergamos 3 curvas $A_{1}, A_{2}, A_{3}$ que são respectivamente o conjunto de pontos fixos das involuções $n_{1}, n_{2}, n_{3}$ que geram o grupo $M_{3}$ (aqui identificamos as componentes dos dois tipos). Mostramos que $M_{3}$ não age discretamente numa componente se a componente não é suave (veja observação 1) ou se um par de tais curvas se interceptam na componente. Finalmente, descrevemos todas as componentes onde $M_{3}$ age discretamente (veja Teorema 1) : para quaisquer $\left.a_{0}, a_{1}, a_{2}, a_{3} \in\right] 0, \pi\left[\right.$, a menos que $a_{0}=a_{1}=a_{2}=a_{3}=\frac{\pi}{2}$ (o caso onde $S\left(a_{0}, a_{1}, a_{2}, a_{3}\right)$ é não suave), existe uma componente (de um dado tipo) onde $M_{3}$ age discretamente se e somente se $a_{j}+a_{k} \leqslant \pi$ para todo $j \neq k$ ou $\pi \leqslant a_{j}+a_{k}$ para todo $j \neq k$, e tal componente é única. Tomando um triângulo ideal no plano hiperbólico e o grupo gerado pelas reflexões em seus lados $A_{1}, A_{2}, A_{3}$, ganhamos uma imagem topológica adequada da ação de $M_{3}$ 
na componente em questão.

Vale mencionar que qualquer componente de $S\left(a_{0}, a_{1}, a_{2}, a_{3}\right)$ é uma superfície em $\mathbb{R}^{3}$ dada por uma das equações (2.3.3) ou (2.3.4).

\subsubsection{Trabalhos relacionados}

Voltando ao exemplo1, levando em conta as equações (2.3.3) e (2.3.4), $a_{1},=a_{2}=a_{3}:=\frac{\pi}{2}$, e fazendo a mundaça de variáveis $x_{j}:= \pm 2 s\left(2 t_{j}-1\right)$, chegamos à equação $x_{1}^{2}+x_{2}^{2}+x_{3}^{2}-x_{1} x_{2} x_{3}-$ $2=t$, onde $s:=\sin a_{0}, t:=-\operatorname{tr} R_{c, k}, k:=e^{a_{0} i}$, e $R_{c, k} \in \mathrm{SU}(1,1)$ (veja a definição no início da Seção 2.2) representa a rotação em sentido anti-horário por $2 a_{0}$ em torno de $c$ no plano hiperbólico. Em outras palavras, nosso grupo $M_{3}$ neste caso é comensurável ao grupo $\Gamma$ de [Gol] no caso $|t|<2$. Se admitirmos no toy example uma isometria parabólica ou hiperbólica no lugar de $R_{c, k}$, poderiamos de fato lidar com o caso geral estudo por by W. M. Goldman [Gol] (a isometria parabólica corresponderia ao caso estudado por P. Waterman e S. Wolpert [WaW]).

A condição $\sum_{j=0}^{n} a_{j}>2 \pi$ (ou $\sum_{j=0}^{n} a_{j}<2 \pi$ ) nas curvaturas dos pontos cônicos, necessária para a existência de uma 2 -esfera hiperbólica (respectivamente, esférica) $\Sigma$ com as singularidades indicadas, é claramente suficiente. Ainda mais, A. D. Alexandrov provou que, para qualquer tal $\Sigma$, existe um único poliedro convexo compacto $P$ no 3-espaço hiperbólico real de curvatura -1 (respectivamente, na 3 -esfera redonda de curvatura 1) cujo bordo $\partial P$ é isométrico a $\Sigma$ com respeito à métrica induzida em $\partial P$ (veja [Ale, 3.6.4, p. 190] para a unicidade e [Ale, 5.3.1, p. 261] para a existência).

G. Mondello e D. Panov [MPa] encontraram condições necessárias e quase suficientes para a existência de uma 2-esfera esférica com pontos cônicos de dados ângulos, cada qual permitido a exceder $2 \pi$. O espaço correspondente pode ser bastante útil quando lidamos com o problema 1. No contexto de poliedros convexos no 3-espaço hiperbólico real, C. D. Hodgson e I. Rivin [HRi] descreveram todas 2-esferas esféricas com todos ângulos cônicos $>2 \pi$ e todas geodésicas fechadas de comprimento $>2 \pi$.

\subsection{Fatos preliminares}

Nós iremos utilizar as seguintes convenções e notação similar às encontradas em [AGr].

Seja $\sigma= \pm 1$. No que se segue, $V$ denota um espaço $\mathbb{C}$-linear 2-dimensional munido de uma forma hermitiana $\langle-,-\rangle$ de assinatura $\left(1+\frac{\sigma+1}{2}, \frac{\sigma-1}{2}\right)$. Denotamos $\mathrm{B} V:=\left\{p \in \mathbb{P}_{\mathbb{C}} V \mid\right.$ $\langle p, p\rangle>0\}$. Assim B $V$ é o espaço modelo de curvatura $\sigma$, a esfera redonda se $\sigma=1$ e o disco hiperbólico se $\sigma=-1$. De fato, quando $\sigma=-1$, a esfera de Riemann-Poincaré $\mathbb{P}_{\mathbb{C}} V$ é colada de dois discos hiperbólicos $\mathrm{B} V$ and $\mathrm{B}^{\prime} V:=\left\{p \in \mathbb{P}_{\mathbb{C}} V \mid\langle p, p\rangle<0\right\}$ ao longo do absoluto $\partial \mathrm{B} V:=\left\{p \in \mathbb{P}_{\mathbb{C}} V \mid\langle p, p\rangle=0\right\}$.

A distância $\operatorname{dist}\left(p_{1}, p_{2}\right)$ entre pontos $p_{1}, p_{2} \in \mathrm{B} V$ é dada pela fórmula $\cos ^{2} \frac{\operatorname{dist}\left(p_{1}, p_{2}\right)}{2}=$ 
$\operatorname{ta}\left(p_{1}, p_{2}\right)$ para $\sigma=1 \mathrm{e} \cosh ^{2} \frac{\operatorname{dist}\left(p_{1}, p_{2}\right)}{2}=\operatorname{ta}\left(p_{1}, p_{2}\right)$ para $\sigma=-1$. Então, a distância é uma função monótona da tancia definida por $\operatorname{ta}\left(p_{1}, p_{2}\right):=\frac{\left\langle p_{1}, p_{2}\right\rangle\left\langle p_{2}, p_{1}\right\rangle}{\left\langle p_{1}, p_{1}\right\rangle\left\langle p_{2}, p_{2}\right\rangle}$.

Para $z \in \mathbb{C} \backslash 0$, denotamos por $\arg z$ e $\operatorname{Arg} z$ as funções argumento tomando valores em ]$-\pi, \pi]$ e em $[0,2 \pi[$, respectivamente.

Fato 1. [AGr, Exemplo 6.1]. A área orientada de um triângulo com vértices dois a dois nãoortogonais $p_{1}, p_{2}, p_{3} \in \mathrm{B} V$ é igual a $\operatorname{area}\left(p_{1}, p_{2}, p_{3}\right)=-2 \sigma \arg \left(g_{12} g_{23} g_{31}\right)$, onde $\left[g_{j k}\right]$ é a matriz de Gram dos $p_{j}$ 's. Se $\sigma=-1$, é possível ver que arg assume valores em $]-\frac{\pi}{2}, \frac{\pi}{2}$ [ quando calculamos a área pela fórmula acima se $\sigma=1$, então arg pode assumir qualquer valor. Em particular, o triângulo é orientado no sentido anti-horário se e somente se $-\sigma \operatorname{Im}\left(g_{12} g_{23} g_{31}\right)>0$.

Para $c \in \mathrm{B} V$ e $k \in \mathbb{C}$ tal que $|k|=1$, a regra $R_{c, k}: p \mapsto(\bar{k}-k) \frac{\langle p, c\rangle}{\langle c, c\rangle} c+k p$ define uma aplicação $\mathbb{C}$-linear $R_{c, k}: V \rightarrow V$.

Fato 2. Sejam $c, p \in \mathrm{B} V$ e sejam $k, k^{\prime} \in \mathbb{C}$ tais que $|k|=\left|k^{\prime}\right|=1$. Então $R_{c, k}$ providencia uma rotação em $\mathrm{B} V$. Mais precisamente, $R_{c, k}$ é a rotação por $\operatorname{Arg} k^{2}$ no sentido anti-horário em torno de $c$. Além disso, $R_{c, k} \in \mathrm{SU} V, R_{c,-k}=-R_{c, k}, R_{c, k} R_{c, k^{\prime}}=R_{c, k k^{\prime}},\left\langle c, R_{c, k} p\right\rangle=k\langle c, p\rangle$, e $\left\langle R_{c, k} p, p\right\rangle=(\operatorname{ta}(p, c)(\bar{k}-k)+k)\langle p, p\rangle$. Se $\sigma=1$ e $\left\langle c, c^{\prime}\right\rangle=0$, então $R_{c, k} R_{c^{\prime}, k}=1$.

Demonstração. Seja $c^{\prime} \in \mathbb{P}_{\mathbb{C}} V$ o ponto ortogonal à $c$ e tomemos representantes tais que $\langle c, c\rangle=1$ $\mathrm{e}\left\langle c^{\prime}, c^{\prime}\right\rangle=\sigma$. Todo ponto $x \in \mathrm{B} V$ pode ser escrito na forma $x=c+\eta c^{\prime}$ para algum único $\eta \in \mathbb{C}$. Quando $\sigma=-1$, temos $|\eta|<1$ e isso corresponde a identificar $\mathrm{B} V$ com o disco unitário em $\mathbb{C}$ centrado na origem; quando $\sigma=1$, identificamos $\mathbb{P}_{\mathbb{C}} V \backslash c^{\prime}$ com $\mathbb{C}$ (em ambos casos, o ponto $c$ é levado para a origem). Nessa identificação, $R_{c, k}$ manda o ponto $x$ para $c+k^{2} \eta c^{\prime}$, i.e., é apenas a multiplicação pelo número complexo unitário $k^{2}$. Na base ortonormal $c, c^{\prime}$, a aplicação $R_{c, k}$ tem a forma $\left[\begin{array}{ll}\bar{k} & 0 \\ 0 & k\end{array}\right]$, e portanto pertence à $\mathrm{SU} V$. As igualdades $R_{c,-k}=-R_{c, k}, R_{c, k} R_{c, k^{\prime}}=R_{c, k k^{\prime}}$, $\left\langle c, R_{c, k} p\right\rangle=k\langle c, p\rangle, \mathrm{e}\left\langle R_{c, k} p, p\right\rangle=(\operatorname{ta}(p, c)(\bar{k}-k)+k)\langle p, p\rangle$ seguem por cálculos automáticos. Finalmente, para mostrar que $R_{c, k} R_{c^{\prime}, k}=1$, é suficiente notar que $R_{c, k} R_{c^{\prime}, k} c=c$ e $R_{c, k} R_{c^{\prime}, k} c^{\prime}=$ $c^{\prime}$.

Seja $\gamma$ uma geodésica em B $V$. Denotamos por $I_{\gamma}$ a reflexão (antiholomorfa) em $\gamma$. Dado um par de geodésicas $\gamma, \gamma^{\prime}$ interceptando em $c \in \mathrm{B} V$, denotamos por $\alpha_{c}\left(\gamma, \gamma^{\prime}\right) \in[0, \pi[$ o ângulo orientado em $c$ de $\gamma$ para $\gamma^{\prime}$ medido no sentido anti-horário.

Fato 3. Seja $1 \neq R \in \mathrm{PU} V$ uma isometria preservando orientação não-trivial de $\mathrm{B} V$. Então existem duas geodésicas distintas $\gamma, \gamma^{\prime}$ tais que $R=I_{\gamma^{\prime}} I_{\gamma}$ em PUV.

As geodésicas são ultraparalelas, tangentes ou concorrentes se e somente se $R$ é respectivamente hiperbólica, parabólica ou elíptica. O par de geodésicas é único a menos de ação do centralizador $C_{R}$ de $R$ em PUV e a ação do centralizador é livre no caso hiperbólico e parabólico. No caso elíptico, o estabilizador do par é dado pela reflexão no ponto de intersecção 
das geodésicas, i.e., por $R_{c, i}$, onde $c$ é o ponto de intersecção. Nesse caso, $I_{\gamma^{\prime}} I_{\gamma}=R_{c, k} \in \mathrm{PU} V$, onde $k:=e^{i \alpha_{c}\left(\gamma, \gamma^{\prime}\right)}$.

Topologicamente, $C_{R}$ é um círculo se $R$ é elíptica e uma linha, se $R$ é hiperbólica ou parabólica.

Fato 4. Seja $\sigma=-1$. Para quaisquer $\left.a_{1}, a_{2} \in\right] 0, \pi\left[\right.$ tais que $a_{1}+a_{2} \neq \pi$, existe um único $t_{0}\left(a_{1}, a_{2}\right)>1 \mathrm{com}$ a propriedade seguinte. Dados dois pontos distintos $q_{1}, q_{2} \in \mathrm{B} V$, denotamos por $\gamma_{1}, \gamma_{2}$ as geodéiscas tais que $q_{j} \in \gamma_{j}, \alpha\left(\gamma, \gamma_{1}\right)=a_{1}$, e $\alpha\left(\gamma_{2}, \gamma\right)=a_{2}$, onde $\gamma$ é a geodésica ligando $q_{1}, q_{2}$. As geodésicas $\gamma_{1}, \gamma_{2}$ se interceptam, são tangentes ou são ultraparalelas se $\operatorname{ta}\left(q_{1}, q_{2}\right)<t_{0}\left(a_{1}, a_{2}\right), \operatorname{ta}\left(q_{1}, q_{2}\right)=t_{0}\left(a_{1}, a_{2}\right)$, ou $\operatorname{ta}\left(q_{1}, q_{2}\right)>t_{0}\left(a_{1}, a_{2}\right)$, respectivamente. No caso $a_{1}+a_{2}=\pi$, as geodésicas $\gamma_{1}, \gamma_{2}$ são sempre ultraparalelas e definimos $t_{0}\left(a_{1}, a_{2}\right):=1$ neste caso.

Fato 5. Sejam $\gamma_{1}, \gamma_{2}$ geodésicas ultraparalelas e sejam $\left.a_{1}, a_{2} \in\right] 0, \pi[$. Então existe uma única geodésica $\gamma$, dependendo suavemente de $\gamma_{1}, \gamma_{2}$, tal que $\alpha\left(\gamma_{1}, \gamma\right)=a_{1}$ e $\alpha\left(\gamma, \gamma_{2}\right)=a_{2}$.

Demonstração. Sejam $v_{1}, v_{1}^{\prime}$ e $v_{2}, v_{2}^{\prime}$ vértices de $\gamma_{1}$ e $\gamma_{2}$ tais que os triângulos $\left(v_{1}^{\prime}, v_{1}, v_{2}\right)$ e $\left(v_{1}^{\prime}, v_{2}, v_{2}^{\prime}\right)$ são ambos orientados no sentido horário. Então o ângulo de $\left[v_{1}^{\prime}, c\right]$ para $\left[c, v_{2}\right]$ cresce monotonicamente de 0 até $\pi$ enquanto $c \in \gamma_{1}$ percorre de $v_{1}$ para $v_{1}^{\prime}$. Em algum $c \in \gamma_{1}$, este ângulo vale $a_{1}$. Similarmente, existe $c^{\prime} \in\left[c, v_{1}^{\prime}\right]$ tal que o ângulo de $\left[v_{1}^{\prime}, c^{\prime}\right]$ para $\left[c^{\prime}, v_{2}^{\prime}\right]$ vale $a_{1}$. Segue então que, para qualquer $p \in\left[c, c^{\prime}\right]$, a geodésica $\gamma_{p}$ que passa por $p$ e que satisfaz $\alpha\left(\gamma_{1}, \gamma_{p}\right)=a_{1}$ intercepta $\gamma_{2}$. Para $p=c$, essa intersecção é $v_{2}$ e, para $p=c^{\prime}$, é $v_{2}^{\prime}$. Logo, o ângulo $\alpha\left(\gamma_{p}, \gamma_{2}\right)$ varia de 0 até $\pi$. Por continuidade, obtemos a existência da $\gamma$ desejada. Se tivermos uma outra $\gamma^{\prime}$, então $\gamma$ e $\gamma^{\prime}$ são ultraparalelas. Então, nós ganhamos um quadrilátero simples cujos ângulos interiores somam $2 \pi$, uma contradição.

Fato 6. Seja $\sigma=-1$ e $\left.a_{0}, a_{1}, a_{2}, a_{3} \in\right] 0, \pi\left[\right.$. Então, para qualquer $t>t_{0}\left(a_{0}, a_{3}\right)$, existe geodésicas geometricamente únicas $\gamma, \gamma_{0}, \gamma_{1}, \gamma_{2}$ tais que $\alpha\left(\gamma_{0}, \gamma_{1}\right)=a_{0}, \alpha\left(\gamma_{1}, \gamma\right)=a_{1}, \alpha\left(\gamma, \gamma_{2}\right)=a_{2}$, $\alpha\left(\gamma_{2}, \gamma_{0}\right)=a_{3}$, and $\operatorname{ta}\left(c_{3}, c_{0}\right)=t$ portanto, $\gamma_{1}, \gamma_{2}$ são ultraparalelas, onde $c_{0}$ e $c_{3}$ são os pontos de intersecção de $\gamma_{0}, \gamma_{1}$ e $\gamma_{0}, \gamma_{2}$, respectivamente. Além disso, as quatro geodésicas dependem suavemente de $t>t_{0}\left(a_{0}, a_{3}\right)$.

Demonstração. Pelo Fato 4, tomando pontos $c_{0}, c_{3}$ com tância ta $\left(c_{0}, c_{3}\right)=t$, ganhamos geodésicas ultraparalelas $\gamma_{1}, \gamma_{2}$ tais que $c_{0} \in \gamma_{1} \operatorname{com} \alpha\left(\gamma_{0}, \gamma_{1}\right)=a_{0}$ e $c_{3} \in \gamma_{2} \operatorname{com} \alpha\left(\gamma_{2}, \gamma_{0}\right)=a_{3}$, onde $\gamma_{0}$ é a geodésica ligando $c_{0}, c_{3}$. Pelo Fato 5, existe uma única geodésica $\gamma$ tal que $\alpha\left(\gamma_{1}, \gamma\right)=a_{1}$ e $\alpha\left(\gamma, \gamma_{2}\right)=a_{2}$.

Fato 7. Seja $\sigma=-1$. Dadas duas geodésicas distintas $\gamma_{1}, \gamma_{2}$ interceptando em algum ponto $c \in \mathrm{B} V$, denotamos $a:=\alpha\left(\gamma_{2}, \gamma_{1}\right)$ e escolhemos $\left.a_{1}, a_{2} \in\right] 0, \pi[$. Então existe um par de geodésicas $\gamma, \gamma^{\prime}$ sujeitas a $\gamma^{\prime}=R_{c, i} \gamma$ tais que $\alpha\left(\gamma_{1}, \gamma\right)=\alpha\left(\gamma_{1}, \gamma^{\prime}\right)=a_{1}$ e $\alpha\left(\gamma, \gamma_{2}\right)=\alpha\left(\gamma^{\prime}, \gamma_{2}\right)=a_{2}$ se e somente se 
- $a+a_{1}+a_{2} \leqslant \pi$, onde o triângulo $\left(c, c_{1}, c_{2}\right)$ é orientado em sentido horário ou degenerado i.e., $c=c_{1}=c_{2}$, ou

- $2 \pi \leqslant a+a_{1}+a_{2}$, quando o triângulo $\left(c, c_{1}, c_{2}\right)$ é orientado em sentido anti-horário ou degenerado,

onde $c_{j}$ é o ponto de intersecção de $\gamma$ e $\gamma_{j}$. Tais geodésicas $\gamma, \gamma^{\prime}$ são únicas quando existem e dependem continuamente de $\gamma_{1}, \gamma_{2}$, e $a$.

A condição $\gamma=\gamma^{\prime}$ é equivalente à $a+a_{1}+a_{2}=\pi$ ou $a+a_{1}+a_{2}=2 \pi$ Equivalentemente, isto significa que $c=c_{1}=c_{2}$.

Fato 8. Seja $\sigma=-1$. Suponhamos que $\left.a_{0}, a_{1}, a_{2}, a_{3} \in\right] 0, \pi\left[\right.$ satisfaça $a_{0}+a_{1}+a_{2}+a_{3}<\pi$ ou $3 \pi<a_{0}+a_{1}+a_{2}+a_{3}$. Então existem geodésicas $\gamma, \gamma_{0}, \gamma_{1}, \gamma_{2}$ tais que $\gamma_{1}, \gamma_{2}$ se interceptam, $\alpha\left(\gamma_{0}, \gamma_{1}\right)=a_{0}, \alpha\left(\gamma_{1}, \gamma\right)=a_{1}, \alpha\left(\gamma, \gamma_{2}\right)=a_{2}$, e $\alpha\left(\gamma_{2}, \gamma_{0}\right)=a_{3}$.

Demonstração. Se $a_{0}+a_{1}+a_{2}+a_{3}<\pi$, então $0<a_{0}+a_{3}<\pi-a_{1}-a_{2}<\pi$. Logo, podemos escolher $a \in] 0, \pi\left[\right.$ tais que $a_{0}+a_{3}<a<\pi-a_{1}-a_{2}$. Portanto, existe um triângulo orientado no sentido anti-horário $\left(c, c_{0}, c_{3}\right)$ com ângulos interiores $\pi-a, a_{0}, a_{3}$, respectivamente. Denote por $\gamma_{0}, \gamma_{1}, \gamma_{2}$ as geodésicas tais que $c_{0}, c_{3} \in \gamma_{0}, c, c_{0} \in \gamma_{1}$, and $c_{3}, c \in \gamma_{2}$. Como $a=\alpha\left(\gamma_{2}, \gamma_{1}\right) \mathrm{e}$ $a+a_{1}+a_{2}<\pi$, pelo Fato 7, existe uma geodésica $\gamma \operatorname{com} \alpha\left(\gamma_{1}, \gamma\right)=a_{1}$ e $\alpha\left(\gamma, \gamma_{2}\right)=a_{2}$.

Se $3 \pi<a_{0}+a_{1}+a_{2}+a_{3}$, então $0<2 \pi-a_{1}-a_{2}<a_{0}+a_{3}-\pi<\pi$. Logo, podemos escolher $a \in] 0, \pi\left[\right.$ tal que $2 \pi-a_{1}-a_{2}<a<a_{0}+a_{3}-\pi$. Portanto, existe um triângulo orientado no sentido horário $\left(c, c_{0}, c_{3}\right)$ com ângulos interiores $a, \pi-a_{0}, \pi-a_{3}$, respectivamente. Denote por $\gamma_{0}, \gamma_{1}, \gamma_{2}$ as geodésicas tais que $c_{0}, c_{3} \in \gamma_{0}, c, c_{0} \in \gamma_{1}$, e $c_{3}, c \in \gamma_{2}$. Como $a=\alpha\left(\gamma_{2}, \gamma_{1}\right) \mathrm{e}$ $2 \pi<a+a_{1}+a_{2}$, pelo Fato 7, existe uma geodésica $\gamma \operatorname{com} \alpha\left(\gamma_{1}, \gamma\right)=a_{1}$ e $\alpha\left(\gamma, \gamma_{2}\right)=a_{2}$.

Fato 9. Sejam $\gamma_{1}, \gamma_{2}$ geodésicas tangentes distintas e seja $\left.a_{1}, a_{2} \in\right] 0, \pi\left[\right.$. Denote por $v_{1}, v$ e por $v, v_{2}$ os vértices de $\gamma_{1}$ e de $\gamma_{2}$, respectivamente. Então existe uma geodésica $\gamma$ tal que $\alpha\left(\gamma_{1}, \gamma\right)=a_{1}$ e $\alpha\left(\gamma, \gamma_{2}\right)=a_{2}$ se e somente se $a_{1}+a_{2}<\pi$ e o triângulo $\left(v_{1}, v, v_{2}\right)$ é orientado em sentido antihorário ou $\pi<a_{1}+a_{2}$ e o triângulo $\left(v_{1}, v, v_{2}\right)$ é orientado em sentido horário. Tal geodésica $\gamma$ é única se existir.

Em particular, qualquer deformação suficientemente pequena de $\gamma_{1}, \gamma_{2}$ ainda permite uma geodésica (um par de geodésicas) com os ângulos requeridos.

Fato 10. Seja $\sigma=-1$. Suponhamos que $\left.a_{0}, a_{1}, a_{2}, a_{3} \in\right] 0, \pi\left[\right.$ satisfazem $a_{0}+a_{3}<\pi<a_{1}+a_{2}$ ou $a_{1}+a_{2}<\pi<a_{0}+a_{3}$. Então existem geodésicas $\gamma, \gamma_{0}, \gamma_{1}, \gamma_{2}$ tais que $\gamma_{1}, \gamma_{2}$ são tangentes, $\alpha\left(\gamma_{0}, \gamma_{1}\right)=a_{0}, \alpha\left(\gamma_{1}, \gamma\right)=a_{1}, \alpha\left(\gamma, \gamma_{2}\right)=a_{2}$, e $\alpha\left(\gamma_{2}, \gamma_{0}\right)=a_{3}$.

Demonstração. Escolhemos um par de geodésicas tangentes distintas $\gamma_{1}, \gamma_{2}$ tais que, em termos do Fato 9, o triângulo $\left(v_{1}, v, v_{2}\right)$ é orientado em sentido horário se $a_{0}+a_{3}<\pi$ e orientado em sentido anti-horário se $\pi<a_{0}+a_{3}$. Pelo Fato 9, existe uma única geodésica $\gamma_{0} \operatorname{com} \alpha\left(\gamma_{2}, \gamma_{0}\right)=a_{3}$ 
e $\alpha\left(\gamma_{0}, \gamma_{1}\right)=a_{0}$. Novamente pelo Fato 9, existe uma única geodésica $\gamma \operatorname{com} \alpha\left(\gamma_{1}, \gamma\right)=a_{1}$ e $\alpha\left(\gamma, \gamma_{2}\right)=a_{2}$ já que $a_{0}+a_{3}<\pi$ implica $\pi<a_{1}+a_{2}$ e $\pi<a_{0}+a_{3}$ implica $a_{1}+a_{2}<\pi$.

Fato 11. Seja $\Sigma$ uma 2-esfera hiperbólica com pontos cônicos $\sigma=-1$ e seja $R \subset \Sigma$ uma região conexa e simplesmente conexa que não contém nenhum ponto cônico. Então existe uma imersão localmente isométrica de $R$ em $\mathrm{B} V$. Se $R$ é uma região do tipo estrela, então a imersão é um mergulho.

\subsection{Lemas técnicos}

Lema 1. Sejam $k_{0}, k_{1}, k_{2}, k_{3} \in \mathbb{C}$ com $\left|k_{j}\right|=1$ fixos. Escolhemos $c_{j} \in \mathrm{B} V, 0 \leqslant j \leqslant 3$, e denota$\operatorname{mos} t_{1}:=\operatorname{ta}\left(c_{0}, c_{1}\right), t_{2}:=\operatorname{ta}\left(R_{c_{1}, k_{1}} c_{0}, c_{2}\right)$, e $t_{3}:=\operatorname{ta}\left(c_{0}, c_{3}\right)$. Se a relação $R_{c_{3}, k_{3}} R_{c_{2}, k_{2}} R_{c_{1}, k_{1}} R_{c_{0}, k_{0}}=$ 1 vale em $\mathrm{SU} V$, então

$$
\operatorname{det}\left[\begin{array}{ccc}
1 & t_{3}\left(k_{3}-\bar{k}_{3}\right)+\bar{k}_{3} & \left(t_{2}\left(\bar{k}_{2}-k_{2}\right)+k_{2}\right) \bar{k}_{0} \\
t_{3}\left(\bar{k}_{3}-k_{3}\right)+k_{3} & 1 & t_{1}\left(k_{1}-\bar{k}_{1}\right)+\bar{k}_{1} \\
\left(t_{2}\left(k_{2}-\bar{k}_{2}\right)+\bar{k}_{2}\right) k_{0} & t_{1}\left(\bar{k}_{1}-k_{1}\right)+k_{1} & 1
\end{array}\right]=0 .
$$

Inversamente, dados $0 \leqslant t_{1}, t_{2}, t_{3} \leqslant 1$ o caso $\sigma=1$ ou $1 \leqslant t_{1}, t_{2}, t_{3}$ o caso $\sigma=-1$ satisfazendo (2.3.1), existem $c_{j}$ 's sujeitos às relações $R_{c_{3}, k_{3}} R_{c_{2}, k_{2}} R_{c_{1}, k_{1}} R_{c_{0}, k_{0}}=1 \mathrm{em} \mathrm{SU} V, t_{1}=\operatorname{ta}\left(c_{0}, c_{1}\right)$, $t_{2}=\operatorname{ta}\left(R_{c_{1}, k_{1}} c_{0}, c_{2}\right)$, e $t_{3}=\operatorname{ta}\left(c_{0}, c_{3}\right)$. Tais $c_{0}, c_{1}, c_{2}, c_{3} \in \mathrm{B} V$ são únicos a menos da ação de $\mathrm{U} V$ se $k_{j} \neq \pm 1$ e $t_{j} \neq 0$ para todo $j$.

Demonstração. Assumimos que $\left\langle c_{j}, c_{j}\right\rangle=1$ para todo $j$. Denotemos $a_{j}:=\sqrt{t}_{j}$ e escolhemos representantes de $c_{1}, c_{3}$ tais que $\left\langle c_{0}, c_{j}\right\rangle=a_{j}$ para todo $j=1,3$. Seja $p_{2}:=R_{c_{3}, k_{3}}^{-1} c_{0}$ e $p_{1}:=$ $R_{c_{1}, k_{1}} c_{0}$. Nós escolhemos um representante de $c_{2}$ tal que $\left\langle p_{1}, c_{2}\right\rangle=a_{2}$. Pelo Fato 2 , a matriz de Gram dos escolhidos $p_{2}, c_{3}, c_{0}, c_{1}, p_{1}, c_{2} \in V$ é

$$
G:=\left[\begin{array}{cccccc}
1 & a_{3} k_{3} & t_{3}\left(k_{3}-\bar{k}_{3}\right)+\bar{k}_{3} & & & \\
a_{3} \bar{k}_{3} & 1 & a_{3} & & & \\
t_{3}\left(\bar{k}_{3}-k_{3}\right)+k_{3} & a_{3} & 1 & a_{1} & t_{1}\left(k_{1}-\bar{k}_{1}\right)+\bar{k}_{1} & \\
& & a_{1} & 1 & a_{1} k_{1} & \\
& & t_{1}\left(\bar{k}_{1}-k_{1}\right)+k_{1} & a_{1} \bar{k}_{1} & 1 & a_{2} \\
& & & & a_{2} & 1
\end{array}\right] .
$$

Como $R_{c_{3}, k_{3}} R_{c_{2}, k_{2}} R_{c_{1}, k_{1}} R_{c_{0}, k_{0}} c_{0}=c_{0}$ é equivalente à $R_{c_{2}, k_{2}} p_{1}=k_{0} p_{2}$ nestes termos, concluímos pelo Fato 2 que

$$
G=\left[\begin{array}{cccccc}
1 & a_{3} k_{3} & t_{3}\left(k_{3}-\bar{k}_{3}\right)+\bar{k}_{3} & * & \left(t_{2}\left(\bar{k}_{2}-k_{2}\right)+k_{2}\right) \bar{k}_{0} & a_{2} \bar{k}_{0} \bar{k}_{2} \\
a_{3} \bar{k}_{3} & 1 & a_{3} & * & * & * \\
t_{3}\left(\bar{k}_{3}-k_{3}\right)+k_{3} & a_{3} & 1 & a_{1} & t_{1}\left(k_{1}-\bar{k}_{1}\right)+\bar{k}_{1} & * \\
& * & a_{1} & 1 & a_{1} k_{1} & * \\
\left(t_{2}\left(k_{2}-\bar{k}_{2}\right)+\bar{k}_{2}\right) k_{0} & * & t_{1}\left(\bar{k}_{1}-k_{1}\right)+k_{1} & a_{1} \bar{k}_{1} & 1 & a_{2} \\
a_{2} k_{0} k_{2} & * & * & * & a_{2} & 1
\end{array}\right] .
$$

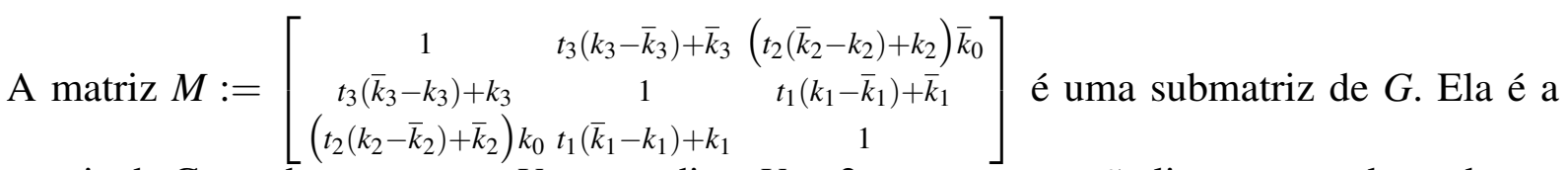
matriz de Gram de $p_{2}, c_{0}, p_{1} \in V ;$ como $\operatorname{dim}_{\mathbb{C}} V=2$, estes pontos são linearmente dependentes e portanto sua matriz de Gram é degenerada. Assim, nós chegamos em (2.3.1). 
De modo a provar a unicidade, nós podemos assumir sem perda de generalidade que os $c_{j}$ 's são normalizados de forma que (2.3.2) é a matriz de Gram de $p_{2}, c_{3}, c_{0}, c_{1}, p_{1}, c_{2}$. Note que os pontos $p_{2}, c_{0}, p_{1} \in V$ cuja matriz de Gram é $M$ são únicos a menos de ação de $\mathrm{U} V$. De fato, a afirmação é simples $t_{j} \neq 1$ para algum $1 \leqslant j \leqslant 3$ porque, nesse caso, $\left|u_{j}\right| \neq 1$, onde $u_{j}:=t_{j}\left(\bar{k}_{j}-k_{j}\right)+k_{j}$. Se $t_{1}=t_{2}=t_{3}=1$, então os pontos $p_{2}, c_{0}, p_{1}$ coincidem em $\mathrm{B} V$ e, novamente, a afirmação segue.

Considere as matrizes de Gram

$$
\begin{gathered}
M_{3}:=\left[\begin{array}{ccc}
1 & a_{3} k_{3} & t_{3}\left(k_{3}-\bar{k}_{3}\right)+\bar{k}_{3} \\
a_{3} \bar{k}_{3} & 1 & a_{3} \\
t_{3}\left(\bar{k}_{3}-k_{3}\right)+k_{3} & a_{3} & 1
\end{array}\right], \quad M_{1}:=\left[\begin{array}{ccc}
1 & a_{1} & t_{1}\left(k_{1}-\bar{k}_{1}\right)+\bar{k}_{1} \\
a_{1} & 1 & a_{1} k_{1} \\
t_{1}\left(\bar{k}_{1}-k_{1}\right)+k_{1} & a_{1} \bar{k}_{1} & 1
\end{array}\right], \\
M_{2}:=\left[\begin{array}{ccc}
1 & \left(t_{2}\left(\bar{k}_{2}-k_{2}\right)+k_{2}\right) \bar{k}_{0} a_{2} \bar{k}_{0} \bar{k}_{2} \\
\left(t_{2}\left(k_{2}-\bar{k}_{2}\right)+\bar{k}_{2}\right) k_{0} & 1 & a_{2} \\
a_{2} k_{0} k_{2} & a_{2} & 1
\end{array}\right]
\end{gathered}
$$

das triplas $p_{2}, c_{3}, c_{0}, c_{0}, c_{1}, p_{1}$, e $p_{2}, p_{1}, c_{2}$. Dados $p_{2}, c_{0}, p_{1} \in V$ com matriz de Gram $M$, o ponto $c_{j} \in V$ é unicamente determinado por $M_{j}$ se $\left|u_{j}\right| \neq 1$. Ainda, $c_{j}$ é unicamente determinado por $M_{j}$ se $a_{j}=1$. Resta observar que $\left|u_{j}\right|=1$ é equivalente à $t_{j}=0,1$ ou $k_{j}= \pm 1$.

Finalmente, vamos mostrar a existência $\operatorname{dos} c_{j}$ 's. Supondo que (2.3.1) seja válida, $\operatorname{det} M=$ 0. Como $\left|u_{j}\right|^{2}-1=2 t_{j}\left(t_{j}-1\right)\left(1-\operatorname{Re} k_{j}^{2}\right)$, temos que $\left|u_{j}\right| \geqslant 1$ se $\sigma=-1$ e $\left|u_{j}\right| \leqslant 1$ se $\sigma=1$. Isto significa que existem pontos $p_{2}, c_{0}, p_{1} \in V$ cuja matriz de Gram é $M$. Estes pontos geram $V$ se $\operatorname{rk} M=2$ e coincidem em $\mathrm{B} V$ se $\operatorname{rk} M=1$. Por uma razão similar, as igualdades $\operatorname{det} M_{3}=$ $\operatorname{det} M_{1}=\operatorname{det} M_{2}=0$ implicam que existem $c_{3}, c_{1}, c_{2}$ tais que $M_{3}, M_{1}, M_{2}$ são as matrizes de Gram das triplas $p_{2}, c_{3}, c_{0}, c_{0}, c_{1}, p_{1}$, e $p_{2}, p_{1}, c_{2}$, respectivamente. De $\left[k_{3} a_{3}\left(1-k_{3}^{2}\right)-1\right] M_{3}=0$, obtemos $k_{3} p_{2}+a_{3}\left(1-k_{3}^{2}\right) c_{3}-c_{0}=0$, i.e., $R_{c_{3}, k_{3}} p_{2}=c_{0}$. De $\left[k_{1} a_{1}\left(\bar{k}_{1}-k_{1}\right)-1\right] M_{1}=0$, concluímos que $k_{1} c_{0}+a_{1}\left(\bar{k}_{1}-k_{1}\right) c_{1}-p_{1}=0$, i.e., $R_{c_{1}, k_{1}} c_{0}=p_{1}$. Segue então de $\left[k_{0}-k_{2} a_{2}\left(k_{2}-\bar{k}_{2}\right)\right] M_{2}=0$ que $k_{0} p_{2}-k_{2} p_{1}+a_{2}\left(k_{2}-\bar{k}_{2}\right) c_{2}=0$, i.e., $R_{c_{2}, k_{2}} p_{1}=k_{0} p_{2}$. Como $R_{c_{0}, k_{0}} c_{0}=\bar{k}_{0} c_{0}$, obtemos $R_{c_{3}, k_{3}} R_{c_{2}, k_{2}} R_{c_{1}, k_{1}} R_{c_{0}, k_{0}} c_{0}=c_{0}$. Levando em conta que $R_{c_{3}, k_{3}} R_{c_{2}, k_{2}} R_{c_{1}, k_{1}} R_{c_{0}, k_{0}} \in \mathrm{SU} V$ e $\left\langle c_{0}, c_{0}\right\rangle \neq$ 0 , nós chegamos à $R_{c_{3}, k_{3}} R_{c_{2}, k_{2}} R_{c_{1}, k_{1}} R_{c_{0}, k_{0}}=1$.

A relação $R_{c_{3}, k_{3}} R_{c_{2}, k_{2}} R_{c_{1}, k_{1}} R_{c_{0}, k_{0}}=1$ em PUV significa $R_{c_{3}, k_{3}} R_{c_{2}, k_{2}} R_{c_{1}, k_{1}} R_{c_{0}, k_{0}}= \pm 1$ no nível de $\mathrm{SU} V$. Dados números complexos unitários $k_{0}, k_{1}, k_{2}, k_{3} \in \mathbb{C}$, os próximos dois lemas tratam das soluções, no nível de $\mathrm{SU} V$, das equações $R_{c_{3}, k_{3}} R_{c_{2}, k_{2}} R_{c_{1}, k_{1}} R_{c_{0}, k_{0}}= \pm 1$. (Note que, pelo Lema 1 e Fato 2, a equação (2.3.3) diz que $R_{c_{3}, k_{3}} R_{c_{2}, k_{2}} R_{c_{1}, k_{1}} R_{c_{0}, k_{0}}=1$ para alguns $c_{0}, c_{1}, c_{2}, c_{3} \in \mathrm{B} V$ enquanto a equação (2.3.4) diz que $R_{c_{3}, k_{3}} R_{c_{2}, k_{2}} R_{c_{1}, k_{1}} R_{c_{0}, k_{0}}=-1$ para alguns $c_{0}, c_{1}, c_{2}, c_{3} \in \mathrm{B} V$.)

Lema 2. Dados $\left.a_{0}, a_{1}, a_{2}, a_{3} \in\right] 0, \pi\left[\right.$, definimos $k_{j}:=e^{a_{j} i}$ e $u_{j}:=t_{j}\left(\bar{k}_{j}-k_{j}\right)+k_{j}$ para todo $0 \leqslant j \leqslant 3$. Então a equação

$$
1+2 \operatorname{Re}\left(\bar{k}_{0} u_{1} u_{2} u_{3}\right)=\left|u_{1}\right|^{2}+\left|u_{2}\right|^{2}+\left|u_{3}\right|^{2}
$$


em $t_{1}, t_{2}, t_{3} \geqslant 1$ não possui soluções com $t_{3}=1$ se $\sum_{j} a_{j} \leqslant \pi$ ou $3 \pi \leqslant \sum_{j} a_{j}$.

Demonstração. Como $u_{3}=\bar{k}_{3}$ quando $t_{3}=1$, a equação toma a forma $2 \operatorname{Re}\left(\bar{k}_{0} \bar{k}_{3} u_{1} u_{2}\right)=\left|u_{1}\right|^{2}+$ $\left|u_{2}\right|^{2}$, que é equivalente à $\left|u_{2}-\bar{u}_{1} k_{0} k_{3}\right|^{2}=0$, i.e., a $u_{2}=\bar{u}_{1} k_{0} k_{3}$. Note que, trocando $a_{j}$ por $\pi-a_{j}$, nós trocamos $k_{j}$ por $-\bar{k}_{j}$ e a última equação se torna $-\bar{u}_{2}=-u_{1} \bar{k}_{0} \bar{k}_{3}$, i.e., se mantém a mesma. Portanto, podemos assumir que $\sum_{j} a_{j} \leqslant \pi$.

Denote $s:=\sin \left(a_{0}+a_{3}\right), b:=\cos \left(a_{0}+a_{3}\right), s_{j}:=\sin a_{j}$, e $b_{j}:=\cos a_{j}$ para $j=1,2$. Segue de $\sum_{j} a_{j} \leqslant \pi$ que $s, s_{1}, s_{2}>0$. Nestes termos, a equação $u_{2}=\bar{u}_{1} k_{0} k_{3}$ toma a forma $-2 s_{2} t_{2} i+b_{2}+s_{2} i=\left(2 s_{1} t_{1} i+b_{1}-s_{1} i\right)(b+s i)$. Logo, $b_{2}=b_{1} b+s_{1} s\left(1-2 t_{1}\right)$ e $s_{2}\left(1-2 t_{2}\right)=$ $b_{1} s+s_{1} b\left(2 t_{1}-1\right)$. Isto significa que $2 t_{1}-1=\frac{b_{1} b-b_{2}}{s_{1} s}$ e $2 t_{2}-1=\frac{b_{2} b-b_{1}}{s_{2} s}$ pois $b^{2}+s^{2}=1$. Como $s_{1} s, s_{2} s>0$, de $t_{1}, t_{2} \geqslant 1$, concluímos que $b_{1} b-s_{1} s \geqslant b_{2}$ e $b_{2} b-s_{2} s \geqslant b_{1}$, i.e., $\operatorname{Re}\left(k_{0} k_{1} k_{3}\right) \geqslant$ $\operatorname{Re} k_{2}$ e $\operatorname{Re}\left(k_{0} k_{2} k_{3}\right) \geqslant \operatorname{Re} k_{1}$. Em vista de $\sum_{j} a_{j} \leqslant \pi$, obtemos $a_{0}+a_{1}+a_{3} \leqslant a_{2}$ e $a_{0}+a_{2}+a_{3} \leqslant$ $a_{1}$, implicando $a_{1}<a_{2}$ e $a_{2}<a_{1}$, uma contradição.

Lema 3. Dados $\left.a_{0}, a_{1}, a_{2}, a_{3} \in\right] 0, \pi\left[\right.$, definimos $k_{j}:=e^{a_{j} i}$ e $u_{j}:=t_{j}\left(\bar{k}_{j}-k_{j}\right)+k_{j}$ para todo $0 \leqslant j \leqslant 3$. Então as soluções da equação

$$
1-2 \operatorname{Re}\left(\bar{k}_{0} u_{1} u_{2} u_{3}\right)=\left|u_{1}\right|^{2}+\left|u_{2}\right|^{2}+\left|u_{3}\right|^{2}
$$

em $t_{1}, t_{2}, t_{3} \geqslant 1$ formam um conjunto compacto.

Demonstração. Tomamos $\sigma=-1$. Seja $t$ a tância que corresponde a distância $d$. Segue de $\cosh ^{2} \frac{\operatorname{dist}\left(p_{1}, p_{2}\right)}{2}=\operatorname{ta}\left(p_{1}, p_{2}\right)$ que $(2 t-1)^{2}$ é a tância que corresponde à distância $2 d$. Consequentemente, $64 t\left(t-\frac{1}{2}\right)^{2}(t-1)+1=\left(2(2 t-1)^{2}-1\right)^{2}$ é a tância que corresponde à distância $4 d$.

Pelo Lema 1 e Fato 2, a equação (2.3.4) diz que, para alguns pontos $c_{0}, c_{1}, c_{2}, c_{3} \in \mathrm{B} V$, a relação $R_{c_{3}, k_{3}} R_{c_{2}, k_{2}} R_{c_{1}, k_{1}} R_{c_{0},-k_{0}}=1$ é válida em $\mathrm{SU} V$, onde $t_{1}:=\operatorname{ta}\left(c_{0}, c_{1}\right)=\operatorname{ta}\left(c_{1}, p_{1}\right)$, $t_{2}:=\operatorname{ta}\left(p_{1}, c_{2}\right)=\operatorname{ta}\left(c_{2}, p_{2}\right), t_{3}:=\operatorname{ta}\left(p_{2}, c_{3}\right)=\operatorname{ta}\left(c_{3}, c_{0}\right), p_{1}:=R_{c_{1}, k_{1}} c_{0}$, e $p_{2}:=R_{c_{3}, k_{3}}^{-1} c_{0}$. Denotando as correspondentes distâncias por $d_{1}, d_{2}, d_{3}$, obtemos $2 d_{1}+2 d_{2} \geqslant \operatorname{dist}\left(p_{2}, c_{0}\right)$. Logo, se $d_{2}=\max \left(d_{1}, d_{2}\right)$, então $4 d_{2} \geqslant \operatorname{dist}\left(p_{2}, c_{0}\right)$. Pelo Fato $2, \operatorname{ta}\left(p_{2}, c_{0}\right)=\left|t_{3}\left(\bar{k}_{3}-k_{3}\right)+k_{3}\right|^{2}=$ $2 t_{3}\left(t_{3}-1\right)\left(1-\operatorname{Re} k_{3}^{2}\right)+1$. Portanto, $32\left(t_{2}-\frac{1}{2}\right)^{2} t_{2}\left(t_{2}-1\right) \geqslant t_{3}\left(t_{3}-1\right)\left(1-\operatorname{Re} k_{3}^{2}\right)$. Já que $\left(t_{2}-\right.$ $\left.\frac{1}{2}\right)^{2} \geqslant t_{2}\left(t_{2}-1\right)$ e $t_{3}\left(t_{3}-1\right) \geqslant\left(t_{3}-1\right)^{2}$, obtemos que $t_{2}-\frac{1}{2} \geqslant\left(t_{3}-1\right)^{\frac{1}{2}}\left(\frac{1-\operatorname{Re} k_{3}^{2}}{32}\right)^{\frac{1}{4}}$, implicando $\max \left(t_{1}, t_{2}\right) \geqslant m\left(t_{3}-1\right)^{\frac{1}{2}}$, onde $m:=\min \left(\left(\frac{1-\operatorname{Re} k_{1}^{2}}{32}\right)^{\frac{1}{4}},\left(\frac{1-\operatorname{Re} k_{2}^{2}}{32}\right)^{\frac{1}{4}},\left(\frac{1-\operatorname{Re} k_{3}^{2}}{32}\right)^{\frac{1}{4}}\right)>0$.

Sem perda de generalidade, nós podemos assumir que $t_{3} \geqslant t_{2} \geqslant t_{1} \geqslant 1$.

Suponha que o conjunto de soluções de (2.3.4) é não compacto. Então existem soluções com $t_{3}$ arbitrariamente grande, i.e., $t_{3} \gg 0$. A desigualdade $\max \left(t_{1}, t_{2}\right) \geqslant m\left(t_{3}-1\right)^{\frac{1}{2}}$ implica $t_{2} \gg 0$.

Denote $s_{j}:=\sin a_{j}, b_{j}:=\cos a_{j}$, e $x_{j}:=s_{j}\left(2 t_{j}-1\right)$. Então $\bar{k}_{0}=b_{0}-s_{0} i, u_{j}=b_{j}-x_{j} i$, e $x_{j} \geqslant s_{j}>0$ pois $t_{j} \geqslant 1$ e $\left.a_{j} \in\right] 0, \pi\left[\right.$. Note que $t_{2} \gg 0$ e $t_{3} \gg 0$ implica $x_{2} \gg 0$ e $x_{3} \gg 0$. Nesses 
termos introduzidos, a equação (2.3.4) toma a forma

$$
\begin{gathered}
1-2 b_{0} b_{1} b_{2} b_{3}+2 s_{0}\left(b_{2} b_{3} x_{1}+b_{3} b_{1} x_{2}+b_{1} b_{2} x_{3}\right)+2 b_{0}\left(b_{3} x_{1} x_{2}+b_{1} x_{2} x_{3}+b_{2} x_{3} x_{1}\right)-2 s_{0} x_{1} x_{2} x_{3}= \\
=b_{1}^{2}+x_{1}^{2}+b_{2}^{2}+x_{2}^{2}+b_{3}^{2}+x_{3}^{2}
\end{gathered}
$$

que pode ser escrita como

$$
x_{1}^{2}+2 p\left(x_{2}, x_{3}\right) x_{1}+q\left(x_{2}, x_{3}\right)=0
$$

onde

$$
\begin{gathered}
p\left(x_{2}, x_{3}\right):=s_{0} x_{2} x_{3}-b_{0} b_{3} x_{2}-b_{0} b_{2} x_{3}-s_{0} b_{2} b_{3}=s_{0}\left(x_{2}-b_{0} b_{2} s_{0}^{-1}\right)\left(x_{3}-b_{0} b_{3} s_{0}^{-1}\right)-b_{2} b_{3} s_{0}^{-1}, \\
q\left(x_{2}, x_{3}\right):=x_{2}^{2}-2 b_{0} b_{1} x_{2} x_{3}+x_{3}^{2}-2 s_{0} b_{1} b_{3} x_{2}-2 s_{0} b_{1} b_{2} x_{3}+2 b_{0} b_{1} b_{2} b_{3}+b_{1}^{2}+b_{2}^{2}+b_{3}^{2}-1 .
\end{gathered}
$$

Segue de $x_{2} \gg 0$ e $x_{3} \gg 0$ que $p\left(x_{2}, x_{3}\right) \gg 0$. Portanto, o fato que a equação (2.3.5) possui raíz $x_{1} \geqslant s_{1}$ implica $s_{1}^{2}+2 p\left(x_{2}, x_{3}\right) s_{1}+q\left(x_{2}, x_{3}\right) \leqslant 0$. Consequentemente, obtemos

$$
\begin{gathered}
s_{1}^{2}+2 s_{0} s_{1} x_{2} x_{3}-2 b_{0} s_{1} b_{3} x_{2}-2 b_{0} s_{1} b_{2} x_{3}-2 s_{0} s_{1} b_{2} b_{3}+ \\
+x_{2}^{2}-2 b_{0} b_{1} x_{2} x_{3}+x_{3}^{2}-2 s_{0} b_{1} b_{3} x_{2}-2 s_{0} b_{1} b_{2} x_{3}+2 b_{0} b_{1} b_{2} b_{3}+b_{1}^{2}+b_{2}^{2}+b_{3}^{2}-1 \leqslant 0
\end{gathered}
$$

que é equivalente a

$$
\begin{gathered}
2\left(1+s_{0} s_{1}-b_{0} b_{1}\right) x_{2} x_{3}+\left(x_{2}-x_{3}\right)^{2}-2\left(b_{0} s_{1}+s_{0} b_{1}\right)\left(b_{3} x_{2}+b_{2} x_{3}\right)+ \\
+2\left(b_{0} b_{1}-s_{0} s_{1}\right) b_{2} b_{3}+b_{1}^{2}+b_{2}^{2}+b_{3}^{2}+s_{1}^{2}-1 \leqslant 0
\end{gathered}
$$

i.e., a

$2\left(1-\operatorname{Re}\left(k_{0} k_{1}\right)\right) x_{2} x_{3}+\left(x_{2}-x_{3}\right)^{2}-2 \operatorname{Im}\left(k_{0} k_{1}\right)\left(b_{3} x_{2}+b_{2} x_{3}\right)+2 \operatorname{Re}\left(k_{0} k_{1}\right) b_{2} b_{3}+b_{2}^{2}+b_{3}^{2} \leqslant 0$.

Mas como $1-\operatorname{Re}\left(k_{0} k_{1}\right)>0$ and $x_{2}, x_{3} \gg 0$, chegamos a uma contradição.

\subsection{0 espaço $S\left(a_{0}, a_{1}, a_{2}, a_{3}\right)$ de hexágonos, $\sigma=-1$}

Nesta seção, nós estudamos em detalhes o espaço $R\left(a_{0}, a_{1}, \ldots, a_{n}\right)$ de relações entre isometrias elípticas como classes de conjugação fixadas para $n=3$.

Daqui em diante, nós assumimos $\sigma=-1$ e as isometrias são consideradas como elementos em PU $V$, ao menos que o contrário seja dito.

Definição 1. Sejam $\left.a_{0}, a_{1}, \ldots, a_{n} \in\right] 0, \pi\left[\right.$ dados. Denotemos por $R:=R\left(a_{0}, a_{1}, \ldots, a_{n}\right)$ o espaço de relações $R_{n} \ldots R_{1} R_{0}=1$ em PUV considerado a menos de conjugação em PUV, onde as classes de conjugação de $R_{0}, R_{1}, \ldots, R_{n} \in \mathrm{PU} V$ são exatamente aquelas de $R_{c, k_{0}}, R_{c, k_{1}}, \ldots, R_{c, k_{n}}$, listadas talvez em ordem diferente, e $k_{j}:=e^{a_{j} i}$. Mesmo que alguns $a_{j}$ 's coincidam, nós ainda consideramos as classes correspondentes como diferentes; em outras palavras, as classes são rotuladas com os $j$ 's. Sem perda de generalidade, assumimos que a classe de conjugação de $R_{0}$ é a de $R_{c, k_{0}}$. 
Podemos ver $R$ como uma variedade relativa de caracteres, i.e., formado por todas PU $V$-representações $\rho: F_{n} \rightarrow$ PU $V$ do grupo livre $F_{n}:=\left\langle r_{0}, r_{1}, \ldots, r_{n} \mid r_{n} \ldots r_{1} r_{0}=1\right\rangle$ de posto $n$ tal que a classe de conjugação de $\rho r_{0}$ é a de $R_{c, k_{0}}$ e a classe de conjugação de $\rho r_{j}$ é a de $R_{c, k_{\beta j}}$ para todo $1 \leqslant j \leqslant n$, onde $\beta$ é uma permutação de $\{1,2, \ldots, n\}$; as representações são consideradas a menos de conjugação em PUV.

Além disso, podemos interpretar $R$ como o espaço de $2 n$-gonos marcados $P$ da maneira seguinte. Seja $\rho: F_{n} \rightarrow$ PUV uma representação como acima e seja $c_{j}$ o ponto fixo de $R_{j}:=\rho r_{j}$. Os vértices consecutivos do caminho geodésico por partes $P$ são $c_{1}, p_{1}, c_{2}, p_{2}, \ldots, c_{n}, p_{n}:=c_{0}$, onde $p_{j}:=R_{j} p_{j-1}$ (os índices são módulo $n$ ). Cada vértice $c_{j}$ é marcado com uma classe de conjugação (de $R_{c, k_{l}}, l \neq 0$ ) e $p_{n}$ é marcada com a classe de conjugação de $R_{c, k_{0}}$.

Definição 2. Denotamos por $m_{0}: F_{n} \rightarrow F_{n}$ o automorfismo de $F_{n}$ dado por $r_{0} \mapsto r_{1} r_{0} r_{1}^{-1}, r_{j} \mapsto$ $r_{j+1}$ para todo $0<j<n$, e $r_{n} \mapsto r_{1}$; para todo $0<l<n$, denotamos por $m_{l}: F_{n} \rightarrow F_{n} \mathrm{o}$ automorfismo de $F_{n}$ dado por $r_{l} \mapsto r_{l+1}, r_{l+1} \mapsto r_{l+1} r_{l} r_{l+1}^{-1}$, e $r_{j} \mapsto r_{j}$ para todo $j \neq l, l+1$; e denotamos por $m_{n}: F_{n} \rightarrow F_{n}$ o automorfismo de $F_{n}$ dado por $r_{0} \mapsto r_{1} r_{n}^{-1} r_{0} r_{n} r_{1}^{-1}, r_{1} \mapsto r_{1} r_{n} r_{1}^{-1}$, $r_{j} \mapsto r_{j}$ para todo $1<j \leqslant n$, e $r_{n} \mapsto r_{1}$. O grupo $\bar{M}_{n}$ gerado por $m_{0}, m_{1}, \ldots, m_{n}$ age pela direita em $R\left(a_{0}, a_{1}, \ldots, a_{n}\right)$ por composição no nível de representações.

Em termos de $2 n$-gonos marcados, a ação de $m_{0}$ é apenas trocar a marca $c_{0}$ de $p_{n}$ para $p_{1}$.

Note que $m_{0} m_{l}=m_{l+1} m_{0}$ para todo $0<l<n$. Além disso. por indução em $l$, o automorfismo $m_{0}^{l}$ aumenta por $l$ (módulo $n$ ) os índices dos $r_{j}$ 's para $1 \leqslant j \leqslant n$ e $m_{0}^{l}: r_{0} \mapsto$ $\left(r_{l} \ldots r_{2} r_{1}\right) r_{0}\left(r_{l} \ldots r_{2} r_{1}\right)^{-1}$. Em particular, $m_{0}^{n}=1$.

Nosso principal interesse nessa seção é entender quando a ação de $\bar{M}_{n}$ numa componente de $R$ é discreta.

Definição 3. Denotamos por $S:=S\left(a_{0}, a_{1}, \ldots, a_{n}\right)$ o quociente de $R\left(a_{0}, a_{1}, \ldots, a_{n}\right)$ pela ação do grupo cíclico de ordem $n$ gerado por $m_{0}$. Em outras palavras, $S$ é formado por $2 n$-gonos com uma marcação esquecida $c_{0}$ nos $p_{j}$ 's.

Sejam $1 \leqslant j, l \leqslant n$ tais que $j \neq l-1, l$ (os índices são módulo $n$ ) e seja $P \in S$ um $2 n$ gono com vértices consecutivos $c_{1}, p_{1}, c_{2}, p_{2}, \ldots, c_{n}, p_{n}$. A modificação $P m_{j, l}$ de $P$ é definida da seguinte forma. O segmento geodésico $\left[p_{j}, c_{l}\right]$ corta $P$ em dois polígonos $P_{1}$ e $P_{2}$ cujos vértices consecutivos são respectivamente $p_{j}, c_{j+1}, p_{j+1}, \ldots, p_{l-1}, c_{l}$ e $c_{l}, p_{l}, c_{l+1}, p_{l+1}, \ldots, c_{j}, p_{j}$. Rodamos $P_{1}$ por $R_{l}$ de tal forma que $R_{l} P_{1}$ e $P_{2}$ se colem ao longo de $\left[c_{l}, p_{l}\right]$ (e removemos este lado), dando origem a um novo $2 n$-gono $P m_{j, l} \in S$ que possui dois novos lados consecutivos, $\left[p_{k}, c_{l}\right]$ e $R_{l}\left[c_{l}, p_{j}\right]=\left[c_{l}, R_{l} p_{j}\right]$.

O grupo $M_{n}$ gerado por todas modificações $m_{j, l}$ age pela direita em $S$. É fácil ver que a aplicação $R \rightarrow S$ é uma bijeção no nível das $\bar{M}_{n}$-orbitas e $M_{n}$-orbitas. (Para uma prova rigorosa, pode-se usar as relações $m_{0} m_{l}=m_{l+1} m_{0}$.) 
Seja $P \in S$ um $2 n$-gono com vértices consecutivos $c_{1}, p_{1}, c_{2}, p_{2}, \ldots, c_{n}, p_{n}$. Então os lados $\left[p_{j-1}, c_{j}\right]$ e $\left[c_{j}, p_{j}\right]$ tem mesmo comprimento e os ângulos 'exteriores' em $c_{j}$, i.e., o ângulo de $\left[c_{j}, p_{j-1}\right]$ até $\left[c_{j}, p_{j}\right]$ em $c_{j}$ medido em sentido anti-horário, é igual a $2 a_{j}$ (a menos que $p_{j-1}=c_{j}=p_{j}$ ). Segue da relação $R_{n} \ldots R_{1} R_{0}=1$ que os ângulos 'interiores' em $p_{j}$ 's somam $2 \pi-2 a_{0}$ módulo $2 \pi$. Inversamente, se um $2 n$-gono $P$ satisfaz as condições listadas, nós obtemos $P \in S$.

Se $a_{j} \geqslant \frac{\pi}{2}$ para todo $0 \leqslant j \leqslant n$ e os ângulos 'interiores' em $p_{j}$ 's somam $2 \pi-2 a_{0}$, então $P$ é convexo. Nesse caso, o $2 n$-gono $P$ permanece convexo depois de qualquer modificação $m \in M_{n}$.

\subsubsection{Pequeno $n$}

Claramente, $S\left(a_{0}\right)=\varnothing$ pois $\left.a_{0} \in\right] 0, \pi\left[\right.$. Pelo Fato 2 , o espaço $S\left(a_{0}, a_{1}\right)$ consiste de apenas um ponto se e somente se $a_{0}+a_{1}=\pi$; caso contrário, $S\left(a_{0}, a_{1}\right)=\varnothing$.

Lema 4. Sejam $\left.a_{0}, a_{1}, a_{2} \in\right] 0, \pi\left[\right.$ fixos. Então $S\left(a_{0}, a_{1}, a_{2}\right)$ é não vazio exatamente nos seguintes casos:

$\bullet \sum_{j} a_{j}=\pi$ ou $\sum_{j} a_{j}=2 \pi$,

$\bullet \sum_{j} a_{j}<\pi$,

- $2 \pi<\sum_{j} a_{j}$.

Nesses casos, $S\left(a_{0}, a_{1}, a_{2}\right)$ consiste de apenas um ponto e o correspondente quadrilátero $\left(c_{1}, p_{1}, c_{2}, p_{2}\right)$ é respectivamente degenerado i.e., $c_{1}=p_{1}=c_{2}=p_{2}$, orientado no sentido horário, e orientado no sentido anti-horário.

Demonstração. Se $c_{0}=c_{1}=c_{2}$, então $k_{0}^{2} k_{1}^{2} k_{2}^{2}=1$ pelo Fato 2 e $\sum_{j} a_{j} \equiv 0 \bmod \pi$. Mais adiante, veremos que o inverso também é verdadeiro.

Não pode ocorrer que apenas dois dos $c_{0}, c_{1}, c_{2}$ coincide. Então, assumimos que estes são todos distintos dois a dois. Denote por $I_{j}$ a reflexão na geodésica $\gamma_{j}$ ligando $c_{j-1}$ e $c_{j}$ (os índices são módulo 3). Então $R_{j}=I_{j+1} I_{j}$. De fato, é suficiente mostrar isto para $j=1$. No quadrilátero $P=\left(c_{1}, p_{1}, c_{2}, p_{2}\right)$, os triângulos $\left(c_{1}, p_{2}, c_{2}\right)$ e $\left(c_{1}, p_{1}, c_{2}\right)$ são congruentes via $I_{2}$ porque $p_{1}=R_{1} p_{2} \neq p_{2}$ em vista de $\left.a_{1} \in\right] 0, \pi$ [ (caso contrário, $R_{1}$ teria dois pontos fixos distintos $c_{1}$ e $p_{2}=c_{0}$ ). Portanto, $I_{2} c_{0}=R_{1} c_{0}$, o que implica que $I_{2} I_{1} c_{0}=R_{1} c_{0}$ e $I_{2} I_{1} c_{1}=R_{1} c_{1}$. As isometrias (que preservam a orientação) $I_{2} I_{1}$ e $R_{1}$ coincidem em dois pontos distintos. São, portanto iguais.

Pelo Fato $3, a_{j}=\alpha\left(\gamma_{j}, \gamma_{j+1}\right)$ é o ângulo orientado de $\gamma_{j}$ para $\gamma_{j+1}$. É fácil ver que, se o triângulo $\left(c_{0}, c_{1}, c_{2}\right)$ é orientado em sentido horário (anti-horário), então $a_{j}$ (respectivamente, $\pi-a_{j}$ ) é o ângulo interior em $c_{j}$. Então, chegamos em $\sum_{j} a_{j}<\pi$ e $2 \pi<\sum_{j} a_{j}$, respectivamente. Obviamente, nessas condições, o quadrilátero $\left(c_{1}, p_{1}, c_{2}, p_{2}\right)$ é geometricamente único. 


\subsubsection{Topologia de $S\left(a_{0}, a_{1}, a_{2}, a_{3}\right)$}

Agora, trocamos a convenção a respeito da marcação dos vértices $c_{j}$ 's de um ponto $P \in S\left(a_{0}, a_{1}, a_{2}, a_{3}\right)$ de tal forma que $c_{j}$ e $R_{j}$ correspondem às classes de conjugação de $R_{c, k_{j}}$ para todo $1 \leqslant j \leqslant 3$. Portanto, temos dois tipos de hexágonos: aqueles com vértices consecutivos $c_{1}, p_{1}, c_{2}, p_{2}, c_{2}, p_{3}$, onde $p_{1}=R_{1} p_{3}, p_{2}=R_{2} p_{1}$, e $p_{3}=R_{3} p_{2}$, e aqueles com vértices consecutivos $p_{3}, c_{3}, p_{2}, c_{2}, p_{1}, c_{1}$, onde $p_{1}=R_{1}^{-1} p_{3}, p_{2}=R_{2}^{-1} p_{1}$, e $p_{3}=R_{3}^{-1} p_{2}$. Para cada hexágono $P$, nós associamos uma tripla $\left(t_{1}, t_{2}, t_{3}\right) \in \mathbb{R}^{3}$, onde $t_{j}:=\operatorname{ta}\left(c_{j}, p_{j}\right)$. No que segue, frequentemente colocaremos a marca $c_{0}$ em $p_{3}$ portanto ganhando a relação $R_{3} R_{2} R_{1} R_{0}=1$ para os hexágonos do primeiro tipo e a relação $R_{0} R_{1} R_{2} R_{3}=1$ para os hexágonos do segundo tipo.

De acordo com a nova convenção, o grupo $M_{3}$, anteriormente gerado por $m_{1,3}, m_{2,1}$, $m_{3,2}$ é agora gerado pelas modificações $n_{1}, n_{2}, n_{3}$ que transformam respectivamente a relação $R_{3} R_{2} R_{1} R_{0}=1$ nas relações $R_{0}^{R_{3}^{-1}} R_{1} R_{2}^{R_{1}^{-1}} R_{3}=1, R_{0}^{R_{3}^{-1}} R_{1}^{R_{2}} R_{2} R_{3}=1, R_{0}^{R_{1}} R_{1} R_{2}^{R_{3}} R_{3}=1$ e a relação $R_{0} R_{1} R_{2} R_{3}=1$ nas relações $R_{3} R_{2}^{R_{1}} R_{1} R_{0}^{R_{3}}=1, R_{3} R_{2} R_{1}^{R_{2}^{-1}} R_{0}^{R_{3}}=1, R_{3} R_{2}^{R_{3}^{-1}} R_{1} R_{0}^{R_{1}^{-1}}=1$, portanto alterando o tipo do hexágono. É fácil verificar que $n_{j}^{2}=1$ para todo $j=1,2,3$ e que $n_{j}$ mantém todos os $t_{l}$ 's exceto talvez $t_{j}$.

Dizemos que um ponto $P \in S\left(a_{0}, a_{1}, a_{2}, a_{3}\right)$ é degenerado com respeito a $t_{3}$ se o único ponto $P^{\prime} \in S\left(a_{0}, a_{1}, a_{2}, a_{3}\right)$ do mesmo tipo que $P$ tal que $t_{3} P^{\prime}=t_{3} P$ é o próprio ponto $P$. Isso implica que $t_{3} P=1$ ou $c_{1}=c_{2}$ em $P$ já que, caso contrário, conseguiríamos outros infinitos pontos com mesmo $t_{3}$ dados por $p_{3}, g c_{1}, g c_{2}, c_{3}$, onde $g \in C_{R}$ percorre o centralizador $C_{R}$ em PUV de $R:=R_{0} R_{3}$ (quando $P$ é do primeiro tipo) ou de $R:=R_{3} R_{0}$ (quando $P$ é do segundo tipo) e a marcação $c_{0}$ é colocada em $p_{3}$. Um ponto $P \in S\left(a_{0}, a_{1}, a_{2}, a_{3}\right)$ é dito ser fronteira com respeito a $t_{3}$ se $P$ é não degenerado com respeito a $t_{3}$ e $t_{3} P=1$. Um ponto $P \in S\left(a_{0}, a_{1}, a_{2}, a_{3}\right)$ é regular com respeito a $t_{3}$ se ele não é degenerado nem de fronteira com respeito a $t_{3}$.

Lema 5. Um ponto $P \in S\left(a_{0}, a_{1}, a_{2}, a_{3}\right)$ é regular com respeito a $t_{3}$ se e somente se $t_{3} P \neq 1 \mathrm{e}$ $c_{1} \neq c_{2}$ em $P$.

A seguir uma lista completa de pontos em $S\left(a_{0}, a_{1}, a_{2}, a_{3}\right)$ degenerados ou de fronteira com respeito a $t_{3}$ :

a. Sejam $\sum_{j} a_{j}<\pi$ ou $3 \pi<\sum_{j} a_{j}$. Então existem exatamente 2 pontos degenerados $P_{1}$ e $P_{2}$ de cada tipo. Estes pontos satisfazem $t_{3} P_{1}=1, c_{1} \neq c_{2}$ para $P_{1}, t_{3} P_{2} \neq 1$, e $c_{1}=c_{2}$ para $P_{2}$.

b. Sejam $\sum_{j} a_{j}=\pi$ ou $\sum_{j} a_{j}=3 \pi$. Então existe exatamente 1 ponto degenerado de cada tipo. Este ponto satisfaz $c_{1}=p_{1}=c_{2}=p_{2}=c_{3}=p_{3}$.

c. Sejam $a_{0}+a_{3}<\pi<a_{1}+a_{2}$ ou $a_{1}+a_{2}<\pi<a_{0}+a_{3}$. Então existe exatamente 1 ponto degenerado $P_{0}$ de cada tipo. A condição $c_{1}=p_{1}=c_{2}=p_{2}=c_{3}=p_{3}$ para $P_{0}$ é equivalente a $\sum_{j} a_{j}=2 \pi$.

d. Sejam $a_{0}+a_{3}=a_{1}+a_{2}=\pi$. Então não existem pontos degenerados. Este é o único caso onde existem pontos de fronteira com respeito a $t_{3}$. Tais pontos, que formam um espaço homeomorfo 
a um raio $\left[1, \infty\left[\right.\right.$, são dados pela condição $t_{3} P=1$.

e. Não existem pontos degenerados nos demais casos, i.e., nos casos

$$
\begin{aligned}
& \text { - } \pi<\sum_{j} a_{j} \operatorname{com} a_{0}+a_{3}<\pi \text { e } a_{1}+a_{2}<\pi, \quad \bullet \sum_{j} a_{j}<3 \pi \text { com } \pi<a_{0}+a_{3} \mathrm{e} \\
& \pi<a_{1}+a_{2}, \\
& \text { - } a_{0}+a_{3}=\pi \operatorname{com} a_{1}+a_{2} \neq \pi \text {, } \\
& \text { - } a_{1}+a_{2}=\pi \operatorname{com} a_{0}+a_{3} \neq \pi \text {. }
\end{aligned}
$$

Demonstração. Se $t_{3} P=1$ e $c_{1} \neq c_{2}$ em $P$, então, pelo Lema $4, P$ é degenerado com respeito a $t_{3}$. Se $t_{3} P \neq 1, c_{1}=c_{2}$ para $P$, e $P^{\prime}$ é um ponto do mesmo tipo que $P$ com $t_{3} P^{\prime}=t_{3} P$, podemos assumir que $P$ e $P^{\prime}$ compartilham os vértices $c_{3}, p_{3}$; então, os vértices $c_{3}, p_{3}, c_{1}=c_{2}$ determinam $P$ e os vértices $c_{3}, p_{3}, c_{1}^{\prime}, c_{2}^{\prime}$ determinam $P^{\prime}$. Colocando a marcação $c_{0}$ em $p_{3}$, ganhamos as relações $\left(R_{2} R_{1}\right) R_{0} R_{3}=1$ para $P$ e $R_{2}^{\prime} R_{1}^{\prime} R_{0} R_{3}=1$ para $P^{\prime}$ no caso do primeiro tipo, e as relações $\left(R_{1} R_{2}\right) R_{3} R_{0}=1$ para $P$ e $R_{1}^{\prime} R_{2}^{\prime} R_{3} R_{0}=1$ para $P^{\prime}$ no caso do segundo tipo. Como $c_{0} \neq c_{3}$ implica $a_{1}+a_{2} \neq \pi$, a relação $R_{2}^{\prime} R_{1}^{\prime}\left(R_{2} R_{1}\right)^{-1}=1$ ou $R_{1}^{\prime} R_{2}^{\prime}\left(R_{1} R_{2}\right)^{-1}=1$, respectivamente, providencia $c_{1}=c_{1}^{\prime}=c_{2}^{\prime}$ pelo Lema 4. Portanto, $P$ é degenerado com respeito a $t_{3}$ se $t_{3} P=1$ e $c_{1} \neq c_{2}$ para $P$ ou se $t_{3} P \neq 1$ e $c_{1}=c_{2}$ para $P$.

Como $t_{3} P \neq 1$ e $c_{1}=c_{2}$ para $P$ implica $a_{1}+a_{2} \neq \pi$, pelo Lema 4, existe um (único) ponto $P \in S\left(a_{0}, a_{1}, a_{2}, a_{3}\right)$ de cada tipo com $t_{3} P \neq 1$ e $c_{1}=c_{2}$ (portanto, degenerado com respeito a $t_{3}$ ) exatamente nos seguintes casos $3 \pi<\sum_{j} a_{j}$

$$
\text { - } \sum_{j} a_{j}<\pi, \quad \bullet \sum_{j} a_{j}<2 \pi \text { e } \pi<a_{1}+a_{2}, \quad \bullet 2 \pi<\sum_{j} a_{j} \text { e } a_{1}+a_{2}<\pi,
$$

Seja $a_{0}+a_{3} \neq \pi$. Novamente pelo Lema 4 , a relação $R_{2} R_{1} R_{0} R_{3}=1$ ou $R_{1} R_{2} R_{3} R_{0}=1$, respectivamente, juntamente com $t_{3} P=1$ determinam unicamente um ponto $P \in S\left(a_{0}, a_{1}, a_{2}, a_{3}\right)$ de cada tipo. Então, $P$ é degenerado com respeito a $t_{3}$ quando $t_{3} P=1$. Além disso, pelo Lema 4 , existe um (único) ponto $P_{0}$ de cada tipo com $t_{3} P_{0}=1$ (e portanto, degenerado com respeito a $t_{3}$ ) exatamente nos seguintes casos

$$
\begin{aligned}
& \bullet \sum_{j} a_{j}=\pi, \quad \bullet \sum_{j} a_{j}=2 \pi \text { e } a_{0}+a_{3} \neq \pi, \quad \bullet \sum_{j} a_{j}=3 \pi, \quad \bullet \sum_{j} a_{j}<\pi, \\
& \bullet \sum_{j} a_{j}<2 \pi \text { e } \pi<a_{0}+a_{3}, \quad \bullet 2 \pi<\sum_{j} a_{j} \text { e } a_{0}+a_{3}<\pi, \quad \bullet 3 \pi<\sum_{j} a_{j} .
\end{aligned}
$$

Note que $c_{1}=c_{2}$ com $t_{3} P=1$ é possível exatamente nos primeiros 3 casos pois $a_{0}+a_{3} \neq \pi$. Nestes casos, $c_{1}=p_{1}=c_{2}=p_{2}=c_{3}=p_{3}$.

No caso $a_{0}+a_{3}=\pi \neq a_{1}+a_{2}$, não existe ponto $P \operatorname{com} t_{3} P=1$.

Seja $a_{0}+a_{3}=a_{1}+a_{2}=\pi$. Então $t_{3} P=1$ implica $c_{0}=c_{3}$ e $c_{1}=c_{2}$. Agora $t_{1} P=t_{2} P$ pode ser um número arbitrário em $\left[1, \infty\left[\right.\right.$. Noutras palavras, a condição $t_{3} P=1$ providencia um ponto de fronteira com respeito a $t_{3}$. Dessa forma, listamos todos os possíveis pontos de fronteira com respeito a $t_{3}$.

Em resumo, chegamos a lista descrita no lema. 
Assumindo que a marcação $c_{0}$ colocada em $p_{3}$, para cada ponto $P \in S\left(a_{0}, a_{1}, a_{2}, a_{3}\right)$, associamos a isometria $R:=R_{0} R_{3}$ se $P$ é do primeiro tipo e a isometria $R:=R_{3} R_{0}$, se $P$ é do segundo tipo. Segue imediatamente do Lema 5 que a isometria $R$ é elíptica (ou idêntica) se $P$ é degenerado ou de fronteira com respeito a $t_{3}$.

Na proposição seguinte, para qualquer ponto $P \in S\left(a_{0}, a_{1}, a_{2}, a_{3}\right)$, colocamos a marcação $c_{0}$ em $p_{3}$.

Proposição 1. Segue uma lista completa de componentes conexas de $S:=S\left(a_{0}, a_{1}, a_{2}, a_{3}\right)$ de dado (cada) tipo apresentadas seguindo os casos listados no Lema 5.

a. Neste caso, existem duas componentes. Uma é topologicamente uma 2-esfera e a isometria $R_{0} R_{3}$ é elíptica para todo ponto nesa componente. A outra componente é topologociamente um plano e a isometria $R_{0} R_{3}$ é hiperbólica para todo ponto nessa componente.

b. Neste caso, existem duas componentes. Uma é um único ponto, degenerado com respeito a $t_{3}$, e a isometria $R_{0} R_{3}$ é elíptica para esse ponto. A outra componente é topologicamente um plano e a isometria $R_{0} R_{3}$ é hiperbólica para todo ponto nessa componente.

c. Neste caso, existe uma única componente, topologicamente um plano. Existem pontos na componente cuja isometria $R_{0} R_{3}$ é elíptica, parabólica e hiperbólica.

d. Neste caso, existem uma única componente, topologicamente um plano. A isometria $R_{0} R_{3}$ é hiperbólica ou idêntica para todo ponto nessa componente.

e. Neste caso, existe uma única componente, topologicamente um plano. A isometria $R_{0} R_{3}$ é hiperbólica para todo ponto nessa componente.

Toda componente $C$, com excessão daquela consistindo de um único ponto, contém uma curva $L_{2} \subset C$ dividindo $C$ em 2 partes tais que $t_{3} C=t_{3} L_{2}$ e toda fibra de $L_{2} \stackrel{t_{3}}{\longrightarrow} \mathbb{R}$ contém no máximo 2 pontos, com a única excessão no caso $\mathbf{d}$ onde a fibra sobre $t_{3}=1$ é topologicamente um raio. A curva $L_{2}$ é topologicamente um círculo ou uma linha quando $C$ é topologicamente uma 2-esfera ou um plano, respectivamente. A curva $L_{2}$ admite uma parametrização suave por $t_{3}$ nos pontos onde a isometria $R_{0} R_{3}$ é hiperbólica.

Demonstração. Seja $C$ uma componente conexa de $S$. Os hexágonoso em $C$ são todos do mesmo tipo. Iremos tratar do primeiro tipo, indicando em parênteses o que acontece no caso do segundo tipo. Denotamos $t_{0}:=t_{0}\left(a_{0}, a_{3}\right)$ (veja Fato 4). Pelo Lema $5, t_{3} P<t_{0}$ se $P \in S$ é degenerado com respeito a $t_{3}$.

Seja $P \in C$. Se $P$ é degenerado com respeito a $t_{3}$, então a fibra de $C \stackrel{t_{3}}{\longrightarrow} \mathbb{R}$ em $P$ consiste de apenas um ponto.

Se $P$ é de fronteira com respeito a $t_{3}$, então a fibra de $C \stackrel{t_{3}}{\longrightarrow} \mathbb{R}$ em $P$ é homeomorfa ao raio $[1, \infty[$ pelo Lema 5 . 
Suponha que $P$ é regular com respeito a $t_{3}$. Denote por $\gamma_{0}$ a geodésica que liga $c_{0} \operatorname{com} c_{3}$. Pelo Fato 3, existem únicas geodésicas $\gamma_{1}, \gamma_{2}$ tais que $R_{0}=I_{1} I_{0}$ e $R_{3}=I_{0} I_{2}$ (tais que $R_{0}=I_{0} I_{1} \mathrm{e}$ $R_{3}=I_{2} I_{0}$ para o segundo tipo) pois $R_{0} \neq 1$ e $R_{3} \neq 1$, onde $I_{j}$ é a reflexão em $\gamma_{j}$. Note que, pelo Fato 3, $\alpha\left(\gamma_{0}, \gamma_{1}\right)=a_{0}$ e $\alpha\left(\gamma_{2}, \gamma_{0}\right)=a_{3}$ (para o segundo tipo, $\alpha\left(\gamma_{1}, \gamma_{0}\right)=a_{0}$ e $\alpha\left(\gamma_{0}, \gamma_{2}\right)=a_{3}$ ).

Como $c_{1}$ e $c_{2}$ não são ambos fixos por $R:=R_{0} R_{3}=I_{1} I_{2} \neq 1$ (por $R:=R_{3} R_{0}=I_{2} I_{1} \neq 1$ para o segundo tipo), os pontos $c_{0}, g c_{1}, g c_{2}, c_{3}$, onde $g$ percorre $C_{R}$, providenciam os hexágonos formando a fibra de $C \stackrel{t_{3}}{\longrightarrow} \mathbb{R}$ e esta fibra é homeomorfa ao centralizador $C_{R}$. De fato, seja $I^{\prime}$ a reflexão numa geodésica ligando $c_{1}$ e $c_{2}$ (aqui admitindo que os pontos $c_{1}$ e $c_{2}$ poderiam coincidir, o que é na verdade impossível). Então, pelo Fato $3, R_{1}=I^{\prime} I_{1}^{\prime}$ e $R_{2}=I_{2}^{\prime} I^{\prime}\left(R_{1}=I_{1}^{\prime} I^{\prime}\right.$ e $R_{2}=I^{\prime} I_{2}^{\prime}$ para o segundo tipo) para certas (únicas) reflexões em geodésicas $I_{1}^{\prime}$ e $I_{2}^{\prime}$. A relação $R_{2} R_{1} R_{0} R_{3}=1$ (a relação $R_{1} R_{2} R_{3} R_{0}=1$ para o segundo tipo) implica $I_{2}^{\prime} I_{1}^{\prime}=I_{2} I_{1} \neq 1$ (implica $I_{1}^{\prime} I_{2}^{\prime}=I_{1} I_{2} \neq 1$ para o segundo tipo). Agora a afirmação segue do Fato 3. De fato, agindo por $C_{R}$, obtemos $I_{1}^{\prime}=I_{1}$ e $I_{2}^{\prime}=I_{2}$ devido ao Fato 3. Como $c_{1} \neq c_{2}$, de $g c_{1}=c_{1}$ e $g c_{2}=c_{2}$ para $g \in C_{R}$, concluímos que $g=1$.

Denote por $L_{2} \subset C$ o subconjunto de todos os pontos $P \in C$ degenerados ou de fronteira com respeito a $t_{3}$ e de todos os pontos $P \in C$ regulares com respeito a $t_{3}$ que satisfazem $I_{1}^{\prime}=I_{1}$ e $I_{2}^{\prime}=I_{2}$. Pelo Fato 3 , a fibra de $C \stackrel{t_{3}}{\longrightarrow} \mathbb{R}$ em $P \in C$ ou é apenas um ponto $P \in L_{2}$ (quando $P$ é degenerado), ou é incluído em $L_{2}$ e homeomorfo a $[1, \infty[$ (quando $P$ é de fronteira), ou é um círculo e contém exatamente dois pontos em $L_{2}$ (quando $P$ é regular e $R$ é elíptica), ou é uma linha e contém exatamente um ponto em $L_{2}$ (quando $R$ é parabólica ou hiperbólica).

Qualquer ponto regular $P \in L_{2}$ pode ser descrito como 4 geodésicas $\gamma, \gamma_{0}, \gamma_{1}, \gamma_{2}$ tais que $c_{0}=\gamma_{1} \cap \gamma_{0}, c_{1}=\gamma \cap \gamma_{1}, c_{2}=\gamma_{2} \cap \gamma, c_{3}=\gamma_{0} \cap \gamma_{2}, \alpha\left(\gamma_{0}, \gamma_{1}\right)=a_{0}, \alpha\left(\gamma_{1}, \gamma\right)=a_{1}, \alpha\left(\gamma, \gamma_{2}\right)=a_{2}$, $\alpha\left(\gamma_{2}, \gamma_{0}\right)=a_{3}, R_{0}=I_{1} I_{0}, R_{1}=I I_{1}, R_{2}=I_{2} I, R_{3}=I_{0} I_{2}$ (para o segundo tipo, $\alpha\left(\gamma_{1}, \gamma_{0}\right)=a_{0}$, $\left.\alpha\left(\gamma, \gamma_{1}\right)=a_{1}, \alpha\left(\gamma_{2}, \gamma\right)=a_{2}, \alpha\left(\gamma_{0}, \gamma_{2}\right)=a_{3}, R_{0}=I_{0} I_{1}, R_{1}=I_{1} I, R_{2}=I I_{2}, R_{3}=I_{2} I_{0}\right)$, onde $I, I_{0}, I_{1}, I_{2}$ são reflexões em geodésicas, respectivamente $\gamma, \gamma_{0}, \gamma_{1}, \gamma_{2}$.

Suponha que exista um ponto $P \in L_{2} \operatorname{com} R$ hiperbólica. Então, pelo Fato 5, podemos deformar continuamente $P$ em $L_{2}$ fazendo $t_{3}$ arbitrariamente grande pois a tância $t_{3}=\operatorname{ta}\left(c_{0}, c_{3}\right)$ é maior ou igual do que a tância entre as geodésicas $\gamma_{1}$ e $\gamma_{2}$. Da mesma maneira, podemos deformar continuamente $P$ diminuindo $t_{3}$ até o momento quando as geodésicas $\gamma_{1}$ e $\gamma_{2}$ se tornam tangentes. Noutras paravras, $] t_{0}, \infty\left[\subset t_{3} C\right.$ se $R$ puder ser hiperbólica em algum ponto em $L_{2}$.

Suponha que exista um ponto $P \in L_{2} \operatorname{com} R$ parabólico. Então, pelo Fato 9, existe uma pequena deformação contínua de $P$ providenciando pontos em $L_{2} \operatorname{com} R$ hiperbólica bem com com $R$ elíptica. Logo, $\left.t_{0} \in\right] b, \infty\left[\subset t_{3} C\right.$ neste caso. Nos termos do Fato 9, se o triângulo $\left(v_{1}, v, v_{2}\right)$ é orientado no sentido horário (anti-horário, no segundo caso) então $a_{0}+a_{3}<\pi$ e $\pi<a_{1}+a_{2}$. Se o triângulo $\left(v_{1}, v, v_{2}\right)$ é orientado no sentido anti-horário (horário, no segundo caso), então $\pi<a_{0}+a_{3}$ e $a_{1}+a_{2}<\pi$. Portanto, um ponto $P \in L_{2}$ com $R$ parabólico pode existir apenas no caso c do Lema 5 . 
Suponha que exista um ponto regular $P \in L_{2} \operatorname{com} R$ elíptico. Denote $a:=\alpha\left(\gamma_{2}, \gamma_{1}\right)$ (denote $a:=\alpha\left(\gamma_{1}, \gamma_{2}\right)$ no segundo caso) e seja $c$ o ponto de intersecção das geodésicas $\gamma_{1}, \gamma_{2}$. Pelo Fato 7 , quando o triângulo $\left(c, c_{0}, c_{3}\right)$ é orientado no sentido horário (anti-horário, no segundo caso), temos $a+\pi<a_{0}+a_{3}$, e quando é orientado no sentido anti-horário (horário, no segundo caso), temos $a_{0}+a_{3}<a$. Novamente pelo Fato 7, obtemos $a+a_{1}+a_{2}<\pi$ ou $2 \pi<a+a_{1}+a_{2}$. Mais uma vez pelo Fato 7 , podemos variar $a$ continuamente pelo intervalo $] 0, \pi[$, assim ganhando pontos em $L_{2}$, se mantermos as duas desigualdades estritas, i.e., as desigualdades em uma das 4 variantes seguintes:

$$
\begin{array}{ll}
\bullet a+\pi \leqslant a_{0}+a_{3} \text { e } a+a_{1}+a_{2} \leqslant \pi, & \bullet a+\pi \leqslant a_{0}+a_{3} \text { e } 2 \pi \leqslant a+a_{1}+a_{2}, \\
\text { - } a_{0}+a_{3} \leqslant a \text { e } a+a_{1}+a_{2} \leqslant \pi, & \bullet a_{0}+a_{3} \leqslant a \text { e } 2 \pi \leqslant a+a_{1}+a_{2} .
\end{array}
$$

(As primeiras duas figuras abaixo ilustram a primeira e a quarta variante; as outras duas ilustram a segunda e a terceira variante.)
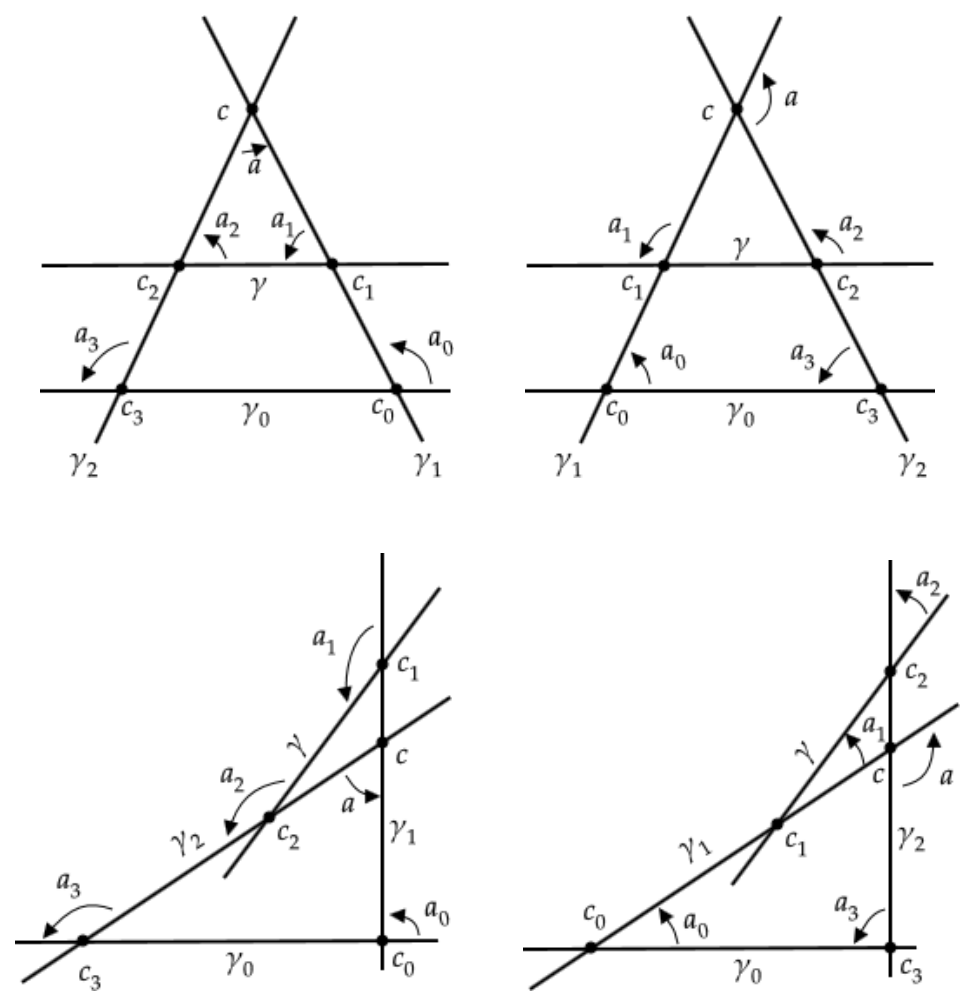

Tal deformação pode ser realizada variando a tância $t_{3} P$ no intervalo $\left[1, t_{0}[\right.$. Note que $a$ depende monotonicamente de $t_{3} P$ enquanto $t_{3} P$ varia no intervalo $] 1, t_{0}\left[\right.$ e $P \in L_{2}$ permanece regular e do mesmo tipo. Reescrevendo as desigualdades de forma mais conveniente, chegamos em

$$
\begin{array}{lr}
\bullet a \leqslant a_{0}+a_{3}-\pi \text { e } a \leqslant \pi-a_{1}-a_{2}, & \bullet 2 \pi-a_{1}-a_{2} \leqslant a \leqslant a_{0}+a_{3}-\pi, \\
\text { - } a_{0}+a_{3} \leqslant a \leqslant \pi-a_{1}-a_{2}, & \bullet a_{0}+a_{3} \leqslant a \text { e } 2 \pi-a_{1}-a_{2} \leqslant a .
\end{array}
$$

Em todas variantes, existe algum $0<a<\pi$ tal que as duas desigualdades são estritas. Isso implica 
- $a_{1}+a_{2}<\pi<a_{0}+a_{3} \operatorname{com} 0<a_{0}+a_{3}-\pi<\pi$ e $0<\pi-a_{1}-a_{2}<\pi$,

- $3 \pi<\sum_{j} a_{j} \operatorname{com} 0<2 \pi-a_{1}-a_{2}<a_{0}+a_{3}-\pi<\pi$,

- $\sum_{j} a_{j}<\pi \operatorname{com} 0<a_{0}+a_{3}<\pi-a_{1}-a_{2}<\pi$,

- $a_{0}+a_{3}<\pi<a_{1}+a_{2} \operatorname{com} 0<a_{1}+a_{2}-\pi<\pi$ e $0<\pi-a_{0}-a_{3}<\pi$.

Em particular, para dados $a_{j}$ 's, apenas uma variante é possível, i.e., as quatro variantes são disjuntas.

Na segunda e terceira variantes, nós estamos no caso a do Lema 5 e podemos atingir ambos valores $2 \pi-a_{1}-a_{2}, a_{0}+a_{3}-\pi$ e $a_{0}+a_{3}, \pi-a_{1}-a_{2}$, respectivamente, variando $a$. Como as desigualdades se tornam igualdades para tais pontos em $L_{2}$, ganhamos pelo Fato 7 os dois pontos degenerados distintos $P_{1}, P_{2}$ mencionados no Lema 5 a. Como $C$ é conexo, nós obtemos $\left[t_{3} P_{1}, t_{3} P_{2}\right] \subset t_{3} C$. Afirmamos que $\left[t_{3} P_{1}, t_{3} P_{2}\right]=t_{3} C$. De fato, caso contrário, existiria um ponto regular extra $P \in L_{2} \operatorname{com} R$ elíptico. Os valores de $a$ para tal $P$ deveriam cair em uma das quatro variantes, e, como estas são disjuntas, a variante deveria permanecer a mesma que começamos. Isso contradiz o fato que $a$ depende monotonicamente de $t_{3} P$. Agora visualizamos $C$ como uma 2-esfera topológica e $L_{2}$, como um círculo topológico dividindo $C$ em duas partes.

Na primeira e quarta variantes, procedemos de maneira similar. Podemos atingir os valores $\min \left(a_{0}+a_{3}-\pi, \pi-a_{1}-a_{2}\right)$ e $\max \left(a_{0}+a_{3}, 2 \pi-a_{1}-a_{2}\right)$, respectivamente, variando a. Como uma das desigualdades se torna uma igualdade para um tal ponto em $L_{2}$, nós obtemos pelo Fato 7 um ponto degenerado $P_{0}$ mencionado no Lema 5 c. Por um argumento similar, $t_{3} C=\left[t_{3} P_{0}, b\right]$ ou $t_{3} C=\left[t_{3} P_{0}, b\left[\right.\right.$ ou $t_{3} C=\left[t_{3} P_{0}, \infty\left[\right.\right.$ para algum $t_{3} P_{0}<b$. No último caso, nós visualizamos $C$ como um plano topológico e $L_{2}$, como uma linha topológica dividindo $C$ em duas partes.

Agora podemos fazer o caso c. Pelo Fato 10, existem geodésicas $\gamma, \gamma_{0}, \gamma_{1}, \gamma_{2}$ tais que $\gamma_{1}, \gamma_{2}$ são tangentes, $\alpha\left(\gamma_{0}, \gamma_{1}\right)=a_{0}, \alpha\left(\gamma_{1}, \gamma\right)=a_{1}, \alpha\left(\gamma, \gamma_{2}\right)=a_{2}$, e $\alpha\left(\gamma_{2}, \gamma_{0}\right)=a_{3}$ (para o segundo tipo, $\alpha\left(\gamma_{0}, \gamma_{2}\right)=a_{3}, \alpha\left(\gamma_{2}, \gamma\right)=a_{2}, \alpha\left(\gamma, \gamma_{1}\right)=a_{1}$, e $\left.\alpha\left(\gamma_{1}, \gamma_{0}\right)=a_{0}\right)$. Denote por $I, I_{0}, I_{1}, I_{2}$ as reflexões em $\gamma, \gamma_{0}, \gamma_{1}, \gamma_{2}$. Pelo Fato 3 , obtemos isometrias elípticas $R_{0}:=I_{1} I_{0}, R_{1}:=I I_{1}, R_{2}:=I_{2} I$, $R_{3}:=I_{0} I_{2}\left(R_{0}:=I_{0} I_{1}, R_{1}:=I_{1} I, R_{2}:=I I_{2}, R_{3}:=I_{2} I_{0}\right.$ para o segundo caso $)$ de classes de conjugação $R_{c, k_{0}}, R_{c, k_{1}}, R_{c, k_{2}}, R_{c, k_{3}}$ sujeitas a $R_{2} R_{1} R_{0} R_{3}=1$ (a $R_{1} R_{2} R_{3} R_{0}=1$ para o segundo tipo) com $R$ parabólica. Então, nós construímos um ponto $P \in S \operatorname{com} R$ parabólica. Este ponto pertence a alguma componente $C$. Portanto, existe um ponto regular $P^{\prime} \in L_{2} \subset C$ com $R$ elíptica. Como vimos anteriormente, $C$ deveria conter o ponto degenerado $P_{0}$ considerado acima. Portanto, tal componente (de dado tipo) é única e nós terminamos o caso c porque já sabemos que a presença de um ponto $P \in L_{2} \operatorname{com} R$ hiperbólica (induzido pela presença de um ponto com $R$ parabólica) implica $] t_{0}, \infty\left[\subset t_{3} C\right.$.

Pelos Fatos 6, 4, e 3, num caso arbitrário a-e, conseguimos de forma similar um ponto $P_{t} \in S$ de dado tipo, dependendo suavemente de $t>t_{0}$, com $R$ hiperbólica tal que $t_{3} P_{t}=t$. Este ponto gera uma componente conexa $C$ de $S$ tal que $P_{t} \in L_{2}$ e nós já sabemos que $\left.t_{3} C \supset\right] t_{0}, \infty[$. 
Logo, pela unicidade do Fato 6, existe uma única componente conexa $C$ de $S$ (de dado tipo) contendo um ponto com $R$ hiperbólica. No caso c, tal componente já havia sido construída acima. No caso d, obtemos $t_{3} C=\left[t_{0}, \infty\left[\right.\right.$ pelo Lema 5 . Em todos os outros casos, temos que $\left.t_{3} C=\right] t_{0}, \infty[$ porque $t_{0} \in t_{3} C$ implicaria na existência de um ponto com $R$ parabólica e nós já vimos que isso é possível apenas no caso c. Além disso, como $P_{t}$ depende suavemente de $t>t_{0}$, a curva $L_{2}$ ganha uma parametrização suave por $t_{3}$ nos pontos com $R$ hiperbólica.

Dessa forma finalizamos os casos b e e. De fato, nestes casos, não pode existir um ponto regular $P \in L_{2}$ com $R$ parabólica ou elíptica já que isso é possível apenas nos casos c e a, respectivamente. Como $t_{3} P^{\prime}<t_{0}$ para qualquer ponto $P^{\prime} \in S$ degenerado com respeito a $t_{3}$, concluímos que, no caso $\mathbf{b}$, existe uma única componente conexa $C$ de $\left.S, t_{3} C=\right] t_{0}, \infty[$, cujos pontos possuem $R$ hiperbólica e uma única componente consistindo de apenas um único ponto degenerado. Similarmente, no caso e, ganhamos uma única componente conexa $C$ de $S$, $\left.t_{3} C=\right] t_{0}, \infty[$, cujos pontos possuem $R$ hiperbólica. Portanto, nos casos b e e, visualizamos a única componente conexa $C$ de $S$ contendo pontos com $R$ hiperbólica como um plano topológico e $L_{2}$, como uma linha topológica dividindo $C$ em duas partes.

Existe uma única componente conexa $C,] 1, \infty\left[\subset t_{3} C\right.$, no caso d. Pelo Lema $5, t_{3} C=$ $\left[1, \infty\left[\right.\right.$. Claramente, $\left(t_{1} P, t_{2} P, t_{3} P\right)$ tende a $\left(t_{1}, t_{2}, 1\right) \operatorname{com} t_{1}=t_{2}$ quando um ponto $P \in C$ tende a um ponto fronteira com respeito a $t_{3}$ porque $c_{0}=c_{3}$ e $c_{1}=c_{2}$ para um ponto fronteira. Dado $\left.t \in\right] 1, \infty[$, existe um único ponto $P_{t} \in L_{2}$, dependendo continuamente de $t$, tal que $t_{3} P_{t}=t$. Qualquer ponto $P \in C \operatorname{com} t_{3} P=t$ tem a forma $P=g P_{t}$ para uma certa (única) isometria $g \in C_{R}$. Note que, para $t$ perto de 1 , temos $t_{1}\left(g P_{t}\right)$ perto de $t_{1}\left(g^{-1} P_{t}\right)$ apenas porque $c_{0}$ e $c_{1}$ são próximos de $P_{t}$. Isto significa que os pontos $g P_{t}$ e $g^{-1} P_{t}$ tendem ao mesmo ponto fronteira quando $g P_{t}$ tende a um. Noutras palavras, na fibra de $t_{3}=1$, dois pontos distintos com $t>1$ tendem a coincidir quando $t$ tende a 1 (a menos que $g$ tenda a 1). Então, no caso d, visualizamos $C$ como um plano topológico e $L_{2}$, como uma linha topológica dividindo $C$ em duas partes.

Finalmente, no caso a, é suficiente construir um ponto regular $P \in S$ com $R$ elíptica pois, como vimos acima, um tal ponto $P$ providencia os dois pontos degenerados $P_{1}$ e $P_{2}$ na componente conexa contendo $P$, implicando a existência e unicidade de uma componente conexa de $S$ cujos pontos possuem $R$ elíptica.

Pelo Fato 8, existem geodésicas $\gamma, \gamma_{0}, \gamma_{1}, \gamma_{2}$ tais que $\gamma_{1}, \gamma_{2}$ interceptam, $\alpha\left(\gamma_{0}, \gamma_{1}\right)=a_{0}$, $\alpha\left(\gamma_{1}, \gamma\right)=a_{1}, \alpha\left(\gamma, \gamma_{2}\right)=a_{2}$, e $\alpha\left(\gamma_{2}, \gamma_{0}\right)=a_{3}$ (para o segundo tipo, $\alpha\left(\gamma_{0}, \gamma_{2}\right)=a_{3}, \alpha\left(\gamma_{2}, \gamma\right)=a_{2}$, $\alpha\left(\gamma, \gamma_{1}\right)=a_{1}$, e $\left.\alpha\left(\gamma_{1}, \gamma_{0}\right)=a_{0}\right)$. Denote por $I, I_{0}, I_{1}, I_{2}$ a reflexão em $\gamma, \gamma_{0}, \gamma_{1}, \gamma_{2}$. Pelo Fato 3 , as isometrias $R_{0}:=I_{1} I_{0}, R_{1}:=I I_{1}, R_{2}:=I_{2} I, R_{3}:=I_{0} I_{2}$ (as isometrias $R_{0}:=I_{0} I_{1}, R_{1}:=I_{1} I$, $R_{2}:=I I_{2}, R_{3}:=I_{2} I_{0}$ para o segundo tipo) de classes de conjugação $R_{c, k_{0}}, R_{c, k_{1}}, R_{c, k_{2}}, R_{c, k_{3}}$ sujeitas a $R_{2} R_{1} R_{0} R_{3}=1$ (a $R_{1} R_{2} R_{3} R_{0}=1$ para o segundo tipo) providenciam um ponto regular $P \in S$ com $R$ elíptica.

Para dados $\left.a_{0}, a_{1}, a_{2}, a_{3} \in\right] 0, \pi\left[\right.$, denotamos $k_{j}:=e^{a_{j} i} \neq \pm 1$ e $u_{j}:=t_{j}\left(\bar{k}_{j}-k_{j}\right)+k_{j}$. 
Seja $C \subset S:=S\left(a_{0}, a_{1}, a_{2}, a_{3}\right)$ uma componente conexa de $S$ e seja $P \in S$. Então, colocando a marca $c_{0}$ em $p_{3}$, a relação $R_{c_{3}, k_{3}} R_{c_{2}, k_{2}} R_{c_{1}, k_{1}} R_{c_{0}, k_{0}}=1$ vale em PU $V$ se $P$ é do primeiro tipo e a relação $R_{c_{1}, k_{1}} R_{c_{2}, k_{2}} R_{c_{3}, k_{3}} R_{c_{0}, k_{0}}=1$ vale em PUV se $P$ é do segundo tipo. No nível de $\mathrm{SU} V$, obtemos respectivamente as relações $R_{c_{3}, k_{3}} R_{c_{2}, k_{2}} R_{c_{1}, k_{1}} R_{c_{0}, k_{0}}= \pm 1$ e $R_{c_{1}, k_{1}} R_{c_{2}, k_{2}} R_{c_{3}, k_{3}} R_{c_{0}, k_{0}}=$ $\pm 1 \mathrm{em} \mathrm{SU} V$. Estas podem ser reescritas como as relações $R_{c_{3}, k_{3}} R_{c_{2}, k_{2}} R_{c_{1}, k_{1}} R_{c_{0}, \pm k_{0}}=1 \mathrm{e}$ $R_{c_{1}, k_{1}} R_{c_{2}, k_{2}} R_{c_{3}, k_{3}} R_{c_{0}, \pm k_{0}}=1$ em SUV. Pelo Lema 1, chegamos a equação (2.3.3) (quando $\pm k_{0}=k_{0}$ ) ou a equação (2.3.4) (quando $\pm k_{0}=-k_{0}$ ) independentemente do tipo de $P$. Isso significa que $C$ satisfaz a equação (2.3.3) ou a equação (2.3.4).

O corólário seguinte afirma o contrário, i.e., que as componentes de $S$ são dadas pelo tipo do hexágono e por uma das equações (2.3.3) ou (2.3.4).

Corolário 1. Seja $\left.a_{0}, a_{1}, a_{2}, a_{3} \in\right] 0, \pi\left[\right.$. Denotamos $k_{j}:=e^{a_{j} i}$ e $u_{j}:=t_{j}\left(\bar{k}_{j}-k_{j}\right)+k_{j}$.

Para qualquer tipo de hexágono, as soluções da equação (2.3.3) em $t_{1}, t_{2}, t_{3} \geqslant 1$ formam uma componente conexa $C_{1}$ de $S:=S\left(a_{0}, a_{1}, a_{2}, a_{3}\right)$ do primeiro tipo, topologicamente um plano, com isometria hiperbólica $R_{0} R_{3}$ para algum ponto na componente.

A equação (2.3.4) em $t_{1}, t_{2}, t_{3} \geqslant 1$ não possui soluções nos casos $\mathbf{c}-\mathbf{e}$ do Lema 3.4.7. No caso $\mathbf{b}$, a equação (2.3.4) em $t_{1}, t_{2}, t_{3} \geqslant 1$ tem uma única solução que constitui uma componente conexa $C_{0}$ de $S$ de cada tipo dado e a isometria $R_{0} R_{3}$ é elíptica para esse ponto. No caso a, as soluções da equação (2.3.4) em $t_{1}, t_{2}, t_{3} \geqslant 1$ formam uma componente conexa $C_{0}$ de $S$ de cada tipo dado, topologicamente uma 2-esfera, e a isometria $R_{0} R_{3}$ é elíptica para todo ponto nesta componente.

O exposto acima é uma lista completa das componentes conexas de $S$.

Demonstração. Observamos primeiro que um ponto $P \in S$ degenerado com respeito a $t_{3}$ não satisfaz a equação (2.3.3) nos casos a-b. De fato, se $t_{3} P=1$, a afirmação é apenas o Lema 2. Se $c_{1}=c_{2}$, podemos tratar o ponto $P$ como um ponto no espaço $S^{\prime}:=S\left(a_{2}, a_{3}, a_{0}, a_{1}\right)$ : se $P$ corresponde à relação $R_{3} R_{2} R_{1} R_{0}=1$ (à relação $R_{0} R_{1} R_{2} R_{3}=1$ para o segundo tipo), então $P^{\prime}$ corresponde à relação $R_{1} R_{0} R_{3} R_{2}=1$ (à relação $R_{2} R_{3} R_{0} R_{1}=1$ para o segundo tipo), e a afirmação segue do Lema 2 aplicado a $S^{\prime}$, onde $t_{3} P^{\prime}$ se torna 1 , pois a equação (2.3.3) e os casos a-b se mantém os mesmo para $S^{\prime}$.

Num caso arbitrário a-e, existe uma única componente conexa $C_{1}$ de $S$ (de cada tipo dado) possuíndo um ponto com $R_{0} R_{3}$ hiperbólica. Tal componente $C_{1}$ não pode ser compacta já que $] t_{0}, \infty\left[\subset t_{3} C_{1}\right.$. Pelo Lema $3, C_{1}$ deve satisfazer a equação (2.3.3). Pela Proposição 1, existe uma única componente conexa (de cada tipo dado) nos casos c-e; então, nós terminamos estes casos.

Nos casos seguintes a-b, existem duas componentes conexas $C_{0}, C_{1}$ (de cada tipo dado) e $C_{0}$, a componente compacta, não satisfaz a equação (2.3.3) pois, pelo Lema 5, ela contém um ponto $P$ degenerado com respeito a $t_{3}$. Por outro lado, a componente $C_{1}$ contendo um ponto com 
$R_{0} R_{3}$ hiperbólica não pode ser compacta pois $] t_{0}, \infty\left[\subset t_{3} C_{1}\right.$, e portanto, $C_{1}$ não pode satisfazer a equação (2.3.4) pelo Lema 3. Consequentemente, $C_{0}$ satisfaz (2.3.4) e $C_{1}$ satisfaz (2.3.3)

Nós já sabemos (veja o segundo parágrafo em 2.4.2) que o grupo $M_{3}$ é gerado pelas modificações $n_{1}, n_{2}, n_{3}$ e que $n_{j}$ altera o tipo do hexágono e mantém $t_{l}$ quando $l \neq j$. Escrevemos as equações (2.3.3) e (2.3.4) na forma $e_{1}\left(t_{1}, t_{2}, t_{3}\right)=0$ e $e_{0}\left(t_{1}, t_{2}, t_{3}\right)=0$, respectivamente. Como $n_{j}$ transforma a relação $R_{c_{3}, k_{3}} R_{c_{2}, k_{2}} R_{c_{1}, k_{1}} R_{c_{0}, k_{0}}=1$ em SUV na relação $R_{c_{1}, k_{1}} R_{c_{2}, k_{2}} R_{c_{3}, k_{3}} R_{c_{0}, k_{0}}=$ 1 em SUV e vice-versa (analogamente, para a relação $R_{c_{3}, k_{3}} R_{c_{2}, k_{2}} R_{c_{1}, k_{1}} R_{c_{0},-k_{0}}=1$ e SUV), essa modificação preserva as equações $e_{l}\left(t_{1}, t_{2}, t_{3}\right)=0$ pelo Lema 1 . É fácil ver que $e_{l}\left(t_{1}, t_{2}, t_{3}\right)=$ $4 t_{j}^{2} \sin ^{2} a_{j}+$ termos menores em $t_{j} \operatorname{com} \sin a_{j} \neq 0$, i.e., a equação $e_{l}\left(t_{1}, t_{2}, t_{3}\right)=0$ é quadrática em $t_{j}$. Portanto, no nível dos $t_{k}$ 's, a modificação $n_{j}$ apenas permuta as raízes de $e_{l}\left(t_{1}, t_{2}, t_{3}\right)=0$ em $t_{j}$. No que segue, não iremos mais distinguir os tipos dos hexágonos e dessa forma iremos identificar os hexágonos de tipos diferentes com mesmos $\left(t_{1}, t_{2}, t_{3}\right)$. Dessa forma, $n_{j}$ age como uma involução em cada componente $C_{l}$ de $S$, onde $C_{l}$ é agora dada pela equação $e_{l}\left(t_{1}, t_{2}, t_{3}\right)=0$. (Talvez fosse mais correto passar para o subgrupo de índice $2 M$ de $M_{3}$. No entanto, é mais conveniente lidar com involuções e isto não faz nenhuma diferença quanto ao problema de discretude da ação de $M_{3}$ em $S$.)

Definição 4. Seja $1 \leqslant j \leqslant 3$. Denote por $A_{j} \subset S:=S\left(a_{0}, a_{1}, a_{2}, a_{3}\right)$ o conjunto de pontos fixos da involução $n_{j}$. Pelo exposto acima, na componente conexa $C_{l}$ de $S$ dada pela equação $e_{l}\left(t_{1}, t_{2}, t_{3}\right)=$ 0 , o conjunto $A_{j}$ é dado pela equação $\frac{\partial}{\partial t_{j}} e_{l}\left(t_{1}, t_{2}, t_{3}\right)=0$. Em particular, $A_{1} \cap A_{2} \cap A_{3}$ é o conjunto de todos pontos singulares de $S$.

Na prova da proposição 1, colocando a marca $c_{0}$ em $p_{3}$, nós definimos a curva $L_{2} \subset S$ de todos os pontos $P \in S$ degenerados ou fronteira com respeito a $t_{3}$ e de todos pontos $P \in S$ regulares com respeito a $t_{3}$ que estão sujeitos a $R_{0}=I_{1} I_{0}, R_{1}=I I_{1}, R_{2}=I_{2} I, R_{3}=I_{0} I_{2}$ quando $P$ é do primeiro tipo ou sujeitos a $R_{0}=I_{0} I_{1}, R_{1}=I_{1} I, R_{2}=I I_{2}, R_{3}=I_{2} I_{0}$ quando $P$ é do segundo tipo, onde $I, I_{0}, I_{1}, I_{2}$ são reflexões em geodésicas adequadas.

Lema 6. 3.4.11 $A_{2}=L_{2}$.

Demonstração. Se $P \in S$ é degenerado com respeito a $t_{3}$, então $P$ é claramente um ponto fixo de $n_{2}$ pois $P$ é o único ponto em $S$ com o valor $t_{3} P$ para $t_{3}$ e $n_{2}$ mantém o valor de $t_{3}$.

A Definição 4 diz que $P \in A_{2}$ se e somente se $\operatorname{ta}\left(c_{2}, p_{2}\right)=\operatorname{ta}\left(p_{3}, c_{2}\right)$ para $P$. Para um ponto $P \in S$ de fronteira com respeito a $t_{3}$, esta condição é vazia já que $p_{2}=p_{3}$ neste caso.

Então, assumimos que $P \in S$ é regular com respeito a $t_{3}$. Então $c_{0} \neq c_{3}$ (colocamos a marca $c_{0}$ em $\left.p_{3}\right)$ e $c_{1} \neq c_{2}$.

Suponha que $P \in L_{2}$. Então $R_{1}=I I_{1}$ se $P$ é do primeiro tipo e $R_{1}=I_{1} I$ se $P$ é do segundo tipo, onde $I c_{2}=c_{2}, p_{1}=R_{1} p_{3}=I I_{1} c_{0}=I c_{0}$ para $P$ do primeiro tipo, e $p_{1}=R_{1}^{-1} p_{3}=I I_{1} c_{0}=I c_{0}$ para $P$ do segundo tipo. Portanto, $\operatorname{ta}\left(c_{2}, p_{2}\right)=\operatorname{ta}\left(p_{1}, c_{2}\right)=\operatorname{ta}\left(I c_{0}, I c_{2}\right)=\operatorname{ta}\left(p_{3}, c_{2}\right)$, i.e., $P \in A_{2}$. 
Inversamente, seja $P \in A_{2}$, i.e., $\operatorname{ta}\left(c_{2}, p_{2}\right)=\operatorname{ta}\left(p_{3}, c_{2}\right)$. Denote por $I$ e $I_{0}$ as reflexões nas geodésicas ligando $c_{1}, c_{2}$ e $c_{0}, c_{3}$, respectivamente. Já que $\operatorname{ta}\left(c_{0}, c_{1}\right)=\operatorname{ta}\left(c_{1}, p_{1}\right)$ e $\operatorname{ta}\left(p_{1}, c_{2}\right)=$ $\operatorname{ta}\left(c_{2}, p_{2}\right)=\operatorname{ta}\left(c_{0}, c_{2}\right)$, os triângulos $\left(c_{1}, c_{0}, c_{2}\right)$ e $\left(c_{1}, p_{1}, c_{2}\right)$ são congruentes. Como $p_{1}=c_{0}$ implica $c_{0}=c_{1}=p_{1}$ devido a $p_{1}=R_{1} c_{0}$ (quando $P$ é do primeiro tipo) ou $c_{0}=R_{1} p_{1}$ (quando $P$ é do segundo tipo), nós obtemos $p_{1}=I c_{0}$.

Suponha que $c_{2} \neq c_{3}$ e denote por $I_{2}$ a reflexão na geodésica ligando $c_{2}$ e $c_{3}$. Como $\operatorname{ta}\left(c_{2}, p_{2}\right)=\operatorname{ta}\left(c_{0}, c_{2}\right)$ e $\operatorname{ta}\left(p_{2}, c_{3}\right)=\operatorname{ta}\left(c_{3}, c_{0}\right)$, os triângulos $\left(c_{2}, p_{2}, c_{3}\right)$ e $\left(c_{2}, c_{0}, c_{3}\right)$ são congruentes. Como $c_{0}=R_{3} p_{2}$ ou $p_{2}=R_{3} c_{0}$, a igualdade $p_{2}=c_{0}$ implicaria que $p_{2}=c_{0}=c_{3}$. Portanto, $p_{2}=I_{2} c_{0}$ e $p_{2} \neq c_{2}$ pois $p_{2}=c_{2}$ e $\operatorname{ta}\left(c_{2}, p_{2}\right)=\operatorname{ta}\left(c_{0}, c_{2}\right)$ implicariam $p_{2}=c_{0}$.

Seja $P$ do primeiro tipo. Então $R_{3} p_{2}=c_{0}, R_{3} c_{3}=c_{3}, I_{0} I_{2} p_{2}=I_{0} I_{2} I_{2} c_{0}=c_{0}, I_{0} I_{2} c_{3}=c_{3}$, $R_{2} p_{1}=p_{2}, R_{2} c_{2}=c_{2}, I_{2} I p_{1}=I_{2} I I c_{0}=I_{2} c_{0}=p_{2}, I_{2} I c_{2}=c_{2}$ com $c_{0} \neq c_{3}$ e $p_{2} \neq c_{2}$. Logo, $R_{3}=I_{0} I_{2}$ e $R_{2}=I_{2} I$. Escrevendo $R_{0}$ como $R_{0}=I_{1} I_{0}$, onde $I_{1}$ é uma reflexão numa geodésica adequada, concluímos pela relação $R_{3} R_{2} R_{1} R_{0}=1$ que $R_{1}=I I_{1}$. Portanto, $P \in L_{2}$.

Seja $P$ do segundo tipo. Então $R_{3} c_{0}=p_{2}, R_{3} c_{3}=c_{3}, I_{2} I_{0} c_{0}=I_{2} c_{0}=p_{2}, I_{2} I_{0} c_{3}=c_{3}$, $R_{2} p_{2}=p_{1}, R_{2} c_{2}=c_{2}, I I_{2} p_{2}=I I_{2} I_{2} c_{0}=I c_{0}=p_{1}, I I_{2} c_{2}=c_{2}$ com $c_{0} \neq c_{3}$ e $p_{2} \neq c_{2}$. Logo, $R_{3}=I_{2} I_{0}$ e $R_{2}=I I_{2}$. Escrevendo $R_{0}$ como $R_{0}=I_{0} I_{1}$, onde $I_{1}$ é uma reflexão numa geodésica adequada, concluímos pela relação $R_{1} R_{2} R_{3} R_{0}=1$ que $R_{1}=I_{1} I$. Portanto, $P \in L_{2}$.

Suponha que $c_{2}=c_{3}$ e $c_{0} \neq c_{1}$. Denote por $I_{1}$ a geodésica ligando $c_{0}$ e $c_{1}$.

Se $P$ é do primeiro tipo, então $R_{1}=I I_{1}$ pois $R_{1} c_{0}=p_{1}, R_{1} c_{1}=c_{1}, I I_{1} c_{0}=I c_{0}=p_{1}$, $I I_{1} c_{1}=c_{1}$ com $c_{0} \neq c_{1}$. Temos $R_{2}=I_{2} I$ e $R_{3}=I_{0}^{\prime} I_{2}$, onde $I_{2}$ e $I_{0}^{\prime}$ são reflexões em geodésicas adequadas. Pela relação $R_{3} R_{2} R_{1} R_{0}=1$, nós obtemos $R_{0}=I_{1} I_{0}^{\prime}$, e portanto, $I_{0}^{\prime} c_{0}=c_{0}$. Como $c_{0} \neq c_{3}$ e $I_{0}^{\prime} c_{3}=c_{3}$, concluímos que $I_{0}^{\prime}=I_{0}$. Portanto, $P \in L_{2}$.

Se $P$ é do segundo tipo, então $R_{1}=I_{1} I$ pois $R_{1} p_{1}=c_{0}, R_{1} c_{1}=c_{1}, I_{1} I p_{1}=I_{1} I I c_{0}=c_{0}$, $I_{1} I c_{1}=c_{1} \operatorname{com} c_{0} \neq c_{1}$. Temos $R_{2}=I I_{2}$ e $R_{3}=I_{2} I_{0}^{\prime}$, onde $I_{2}$ e $I_{0}^{\prime}$ são reflexões em geodésicas adequadas. Pela relação $R_{1} R_{2} R_{3} R_{0}=1$, nós obtemos $R_{0}=I_{0}^{\prime} I_{1}$, e portanto, $I_{0}^{\prime} c_{0}=c_{0}$. Como $c_{0} \neq c_{3}$ e $I_{0}^{\prime} c_{3}=c_{3}$, concluímos que $I_{0}^{\prime}=I_{0}$. Portanto, $P \in L_{2}$.

Finalmente, suponha que $c_{0}=c_{1}$ e $c_{2}=c_{3}$. Então $I=I_{0}$. Como $c_{0} \neq c_{3}$, ganhamos $R_{1} R_{0}=1$ e $R_{3} R_{2}=1$. Como $R_{0}=I_{1} I_{0}, R_{3}=I_{0} I_{2}$ para $P$ do primeiro tipo e $R_{0}=I_{0} I_{1}, R_{3}=I_{2} I_{0}$ para $P$ do segundo tipo, onde $I_{1}$ e $I_{2}$ são reflexões em geodésicas adequadas, nós chegamos a $R_{1}=I_{0} I_{1}, R_{2}=I_{2} I_{0}$ e a $R_{1}=I_{1} I_{0}, R_{2}=I_{0} I_{2}$, respectivamente. Portanto, $P \in L_{2}$.

Lema 7. Um ponto $P \in S:=S\left(a_{0}, a_{1}, a_{2}, a_{3}\right)$ pertence a $A_{1} \cap A_{2}$ se e somente se $P$ é degenerado ou de fronteira com respeito a $t_{3}$.

Um ponto $P \in S$ é singular se e somente se $c_{1}=p_{1}=c_{2}=p_{2}=c_{3}=p_{3}$ para $P$. Portanto, $S$ é não suave se e somente se $\sum_{j} a_{j}=2 \pi$. Neste caso, existe um único ponto singular em $S$ ele pertence à componente não compacta $C_{1}$.

Demonstração. Observamos inicialmente que existe no máximo um ponto equidistante de 3 
pontos dois a dois distintos. Claramente, $P \in A_{j}$ se e somente se $c_{j}$ é equidistante de $p_{1}, p_{2}, p_{3}$.

Seja $P \in A_{1} \cap A_{2}$. Se os pontos $p_{1}, p_{2}, p_{3}$ são dois a dois distintos, então $c_{1}=c_{2}$. Se $p_{1}=p_{2}$, então $p_{1}=c_{2}=p_{2}, \operatorname{logo}, p_{3}=c_{2}$, portanto, $c_{1}=p_{1}=c_{2}$ novamente. Se $p_{1}=p_{3}$, então $p_{1}=c_{1}=p_{3}, \operatorname{logo}, p_{2}=c_{1}$, portanto, $c_{1}=c_{2}$ novamente. Se $p_{2}=p_{3}$, então $c_{3}=p_{3}$. Logo, $P$ é degenerado ou fronteira com respeito a $t_{3}$. É fácil ver que o inverso também é verdade: se $c_{1}=c_{2}$ ou $c_{3}=p_{3}$ para $P$, então $P \in A_{1} \cap A_{2}$.

Nós agora sabemos que $P$ é singular se e somente se $c_{1}=c_{2}$ ou $c_{3}=p_{3}$ e, simultaneamente, $c_{2}=c_{3}$ ou $c_{1}=p_{1}$. Se $c_{1}=c_{2}=c_{3}$, então, colocando a marca $c_{0}$ em $p_{3}$, segue de $R_{3} R_{2} R_{1} R_{0}=1$ ou de $R_{1} R_{2} R_{3} R_{0}=1$ que $c_{0}=c_{1}$ porque $R_{0} \neq 1$. Logo, $c_{1}=p_{1}=c_{2}=p_{2}=$ $c_{3}=p_{3}$. Se $c_{1}=p_{1}=c_{2}\left(\right.$ ou $\left.c_{2}=c_{3}=p_{3}\right)$, então $p_{3}=c_{1}$ (respectivamente, $\left.p_{2}=c_{3}\right)$ e, novamente $c_{1}=p_{1}=c_{2}=p_{2}=c_{3}=p_{3}$. Se $c_{1}=p_{1}$ e $c_{3}=p_{3}$, então $p_{3}=c_{1}$ e $p_{2}=c_{3}$, implicando $c_{1}=p_{1}=c_{2}=p_{2}=c_{3}=p_{3}$ pois $R_{2}$ tem um único ponto fixo.

\subsubsection{Discretude de $M_{3}$}

Colocando a marca $c_{0}$ em $p_{3}$, um hexágono arbitrário $P \in S\left(a_{0}, a_{1}, a_{2}, a_{3}\right)$ do primeiro tipo corresponde à relação $R_{3} R_{2} R_{1} R_{0}=1$. É fácil ver que, o hexágono $P n_{2} n_{1}$ corresponde à relação $R_{3} R_{2}^{\left(R_{1}^{R_{2}}\right)} R_{1}^{R_{2}} R_{0}=1$, i.e, à relação $R_{3} R_{2}^{R_{2} R_{1}} R_{1}^{R_{2} R_{1}} R_{0}=1$. Por indução em $l, P\left(n_{2} n_{1}\right)^{l}$ corresponde à relação $R_{3} R_{2}^{\left(R_{2} R_{1}\right)^{l}} R_{1}^{\left(R_{2} R_{1}\right)^{l}} R_{0}=1$. Já que $R_{0} R_{3}=\left(R_{2} R_{1}\right)^{-1}$, a ação de $n_{2} n_{1}$ preserva as fibras de $S\left(a_{0}, a_{1}, a_{2}, a_{3}\right) \stackrel{t_{3}}{\longrightarrow} \mathbb{R}$.

Em particular, se $P$ é regular com respeito a $t_{3} \operatorname{com} R_{0} R_{3}$ elíptica e a ação de $M_{3}$ na componente conexa contendo $P$ é discreta, então $R_{0} R_{3}$ tem que ser periódica e o período deve ser o mesmo quando nós variamos levemente $t_{3} P$. Isto é impossível pois o triângulo $\left(c_{0}, c, c_{3}\right)$ que corresponde a relação $R_{0}^{-1}\left(R_{0} R_{3}\right) R_{3}^{-1}=1$ (veja, por exemplo, a prova do Lema 4 ) é completamente determinado por seus ãngulos e, portanto, não admite nenhuma variação de $t_{3}$, onde $c$ é o ponto fixo de $R_{0} R_{3}$. Consequentemente, o grupo $M$ não pode agir discretamente numa componente contendo um ponto regular com respeito a $t_{3} \operatorname{com} R_{0} R_{3}$ elíptica. (Denotamos por $M$ o subgrupo de índice 2 do grupo $M_{3}$.)

Lema 8. Se $M$ age discretamente numa componente conexa de $S\left(a_{0}, a_{1}, a_{2}, a_{3}\right)$, então a componente é a não-compacta (nós ignoramos a componente de apenas um ponto) e

- $a_{j}+a_{k} \leqslant \pi$ para todos $j \neq k$ ou $\pi \leqslant a_{j}+a_{k}$ para todo $j \neq k$.

Demonstração. Suponha que $a_{j}+a_{k}<\pi<a_{l}+a_{m} \operatorname{com} j \neq k$ e $l \neq m$. Assumindo sem perda de generalidade que $a_{0} \leqslant a_{3} \leqslant a_{1} \leqslant a_{2}$, obtemos que $a_{0}+a_{3}<\pi<a_{1}+a_{2}$, i.e., nós estamos no caso c. Pela Proposição 1, nós ganhamos uma única componente. Esta possui um ponto regular com respeito a $t_{3} \operatorname{com} R_{0} R_{3}$ elíptica. Como vimos acima, $M$ não pode agir discretamente nesta componente. 
Na sequência, nós lidaremos apenas com os casos listados no Lema ?? e com as componentes conexas não-compactas $C_{1}$.

Pelo lema 7, a componente $C_{1}$ é não-suave apenas no caso $a_{0}=a_{1}=a_{2}=a_{3}=\frac{\pi}{2}$. Como, pelo Lema $7, A_{1} \cap A_{2} \cap C_{1} \neq \varnothing$ apenas no caso d do Lema 5, as curvas $A_{j}$ são duas a duas disjuntas em $C_{1}$ se $C_{1}$ é suave.

Para $1 \leqslant j \leqslant 3$ e $P \in S\left(a_{0}, a_{1}, a_{2}, a_{3}\right)$, denotemos $t_{j}^{\prime} P:=\operatorname{ta}\left(c_{j}, p_{j+1}\right)$ (os índices são considerados módulo 3). Então, a condição $t_{j} P=t_{j}^{\prime} P$ é equivalente a $P \in A_{j}$. Pela Proposição 1 e Lema ??, assumindo $C_{1}$ suave, a curva $A_{j}$ é uma linha suave em $C_{1}$ e divide o plano suave $C_{1}$ em duas partes $H_{j}$ e $H_{j}^{\prime}$ dadas pelas desigualdades $t_{j} \leqslant t_{j}^{\prime}$ e $t_{j} \geqslant t_{j}^{\prime}$, respectivamente.

Lema 9. Suponha que

$\bullet a_{j}+a_{k} \leqslant \pi$ para todo $j \neq k$ ou $\pi \leqslant a_{j}+a_{k}$ para todo $j \neq k$.

Então a componente não-compacta $C_{1}$ de $S\left(a_{0}, a_{1}, a_{2}, a_{3}\right)$, i.e., a componente dada pela equação $e_{1}\left(t_{1}, t_{2}, t_{3}\right)=0$, é suave a menos que $a_{0}=a_{1}=a_{2}=a_{3}=\frac{\pi}{2}$. Assumindo $C_{1}$ suave, as curvas $A_{1}, A_{2}, A_{3}$ em $C_{1}$ são linhas suaves duas a duas disjuntas, cada uma dividindo o plano suave $C_{1}$ em duas partes e, mais ainda, $A_{k} \cap C_{1} \subset H_{j} \cap C_{1}$ para todo $j$ e $k$.

Demonstração. Suponha que, digamos, $A_{2} \cap C_{1} \not \subset H_{1} \cap C_{1}$. Note que as desigualdades $0<a_{j}<\pi$ e $a_{j}+a_{k} \leqslant \pi$ para todo $j \neq k$ define em $\mathbb{R}^{4}$ uma região convexa que se mantém conexa depois da remoção do ponto $\left(\frac{\pi}{2}, \frac{\pi}{2}, \frac{\pi}{2}, \frac{\pi}{2}\right.$ ) (similarmente, para as desigualdades $\pi \leqslant a_{j}+a_{k}$ ). Logo, podemos variar os $a_{j}$ 's mantendo $C_{1}$ suave. Como $A_{1} \cap A_{2} \cap C_{1}=\varnothing$ durante a deformação e a equação $e_{1}\left(t_{1}, t_{2}, t_{3}\right)=0$ bem como as funções $t_{1}^{\prime}, t_{2}^{\prime}$ dependem continuamente dos $a_{j}$ 's, obtemos $A_{2} \cap C_{1} \not \subset H_{1} \cap C_{1}$ para o caso especial de $a_{1}=a_{2}=a_{3}=\frac{\pi}{2}$ e $\left.a_{0} \in\right] 0, \frac{\pi}{2}[$. Neste caso, um hexágono é apenas um triângulo com vértices $p_{1}, p_{2}, p_{3}$ cujos ângulos interiores somam $2 a_{0}$, e $c_{1}, c_{2}, c_{3}$ são respectivamente os pontos médios dos lados $\left[p_{3}, p_{1}\right],\left[p_{1}, p_{2}\right],\left[p_{2}, p_{3}\right]$ do triângulo. Como $A_{2} \cap C_{1} \not \subset H_{1} \cap C_{1}$ implica $A_{2} \cap H_{1} \cap C_{1}=\varnothing$, o triângulo isósceles $\left(p_{1}, p_{2}, p_{3}\right)$ com $\operatorname{dist}\left(p_{1}, p_{3}\right)=\operatorname{dist}\left(p_{2}, p_{3}\right)$ e $\operatorname{dist}\left(p_{1}, p_{2}\right)=2 \operatorname{dist}\left(c_{2}, p_{3}\right)$ cujos ângulos interiores somam $2 a_{0}$ satisfaz $2 \operatorname{dist}\left(c_{1}, p_{2}\right)<\operatorname{dist}\left(p_{1}, p_{3}\right)$, o que é impossível.

Teorema 1. O grupo $M_{3}$ gerado pelas modificações age discretamente numa componente $C$ de $S\left(a_{0}, a_{1}, a_{2}, a_{3}\right)$ exatamente quando $C$ é não-compacta,

- $a_{j}+a_{k} \leqslant \pi$ para todo $j \neq k$ ou $\pi \leqslant a_{j}+a_{k}$ para todo $j \neq k$,

e $a_{j} \neq \frac{\pi}{2}$ para algum $0 \leqslant j \leqslant 3$. Neste caso, o quociente $C / M$ é uma 2 -esfera com 3 buracos e $M$ é o grupo livre de posto 2, onde $M$ é o subgrupo de índice 2 de $M_{3}$.

Demonstração. Suponha que $a_{j} \neq \frac{\pi}{2}$ para algum $0 \leqslant j \leqslant 3$. Pelo Lema ??, é suficiente mostrar que $M_{3}$ age discretamente na componente não-compacta $C_{1}$ nos casos mencionados no teorema. 
Denotemos $T:=H_{1} \cap H_{2} \cap H_{3}$. Pelo Lema 9 e proposição 1, as cópias $T n_{1}, T n_{2}, T n_{3}$ são duas a duas disjuntas interceptam $T$ apenas nas linhas $A_{1}, A_{2}, A_{3}$, respectivamente. Ainda mais, $A_{j}$ vive na intersecção de $T_{1}:=T \cup T n_{1} \cup T n_{2} \cup T n_{3}$ e $T_{1}$ é fechado, conectado, e limitado pelas linhas disjuntas duas a duas $A_{3} n_{1}, A_{2} n_{1}, A_{1} n_{2}, A_{3} n_{2}, A_{2} n_{3}, A_{1} n_{3}$. Aplicando para $T_{1}$ as involuções $n_{1} n_{3} n_{1}, n_{1} n_{2} n_{1}, n_{2} n_{1} n_{2}, n_{2} n_{3} n_{2}, n_{3} n_{2} n_{3}, n_{3} n_{1} n_{3}$ correspondenes à estas linhas, obtemos cópias disjuntas duas a duas de $T_{1}$. Elas interceptam $T_{1}$ apenas nas linhas listadas. Denotemos por $T_{2}$ a união de $T_{1}$ com as cópias listadas de $T_{1}$, e assim por diante. Um argumento padrão irá mostrar que o grupo $M_{3}$ age discretamente em $C_{1}$ e que $T$ é sua região fundamental se observarmos que $D:=\bigcup_{j} T_{j}$ coincide com $C_{1}$.

Como $D$ é $M_{3}$-estável e aberto em $C_{1}$, sua fronteira $\partial D:=\bar{D} \backslash D$ é $M_{3}$-estável e fechada em $\mathbb{R}^{3}$. É suficiente mostrar que $\partial D=\varnothing$.

Suponha que a intersecção $D_{r}$ de $\partial D$ com a bola fechada de raio $r>1$ centrada na origem em $\mathbb{R}^{3}$ é não-vazia. Já que $D_{r}$ é compacto, existe um ponto $P \in D_{r}$ com um valor minimal de $t_{1} t_{2} t_{3}$. Como $T \cap \partial D=\varnothing$, nós temos, digamos, $t_{3}^{\prime} P<t_{3} P$. Então $t_{1}\left(P n_{3}\right)=t_{1} P, t_{2}\left(P n_{3}\right)=t_{2} P$, $t_{3}\left(P n_{3}\right)=t_{3}^{\prime} P<t_{3} P$, e $P n_{3} \in D_{r}$. Mas isso contradiz a escolha de $P$.

$\mathrm{O}$ caso restante $a_{0}=a_{1}=a_{2}=a_{3}=\frac{\pi}{2}$ é considerada na próxima observação.

Observação 1. A componente conexa $C$ de $S:=S\left(\frac{\pi}{2}, \frac{\pi}{2}, \frac{\pi}{2}, \frac{\pi}{2}\right)$ com a ação do grupo $M$ é isomorfa ao espaço $\mathbb{R}^{2} / \pm 1$ com a ação natural do subgrupo de congruência $\Gamma_{2}$ em $\mathrm{SL}_{2} \mathbb{Z}$ que é sabido ser gerado por $\left[\begin{array}{ll}1 & 2 \\ 0 & 1\end{array}\right]$ e $\left[\begin{array}{ll}1 & 0 \\ 2 & 1\end{array}\right]$. Portanto, quase toda $M$-órbita é densa em $C$.

Demonstração. Todo vértice de um hexágono arbitrário $P \in S$ vive numa geodésica. Logo, vendo um hexágono com uma tripla $\left(c_{1}, c_{2}, c_{3}\right)$, podemos interprear isso como uma tripla de números reais considerados a menos de isometrias da reta real. Nestes termos, $R_{j}: r \mapsto 2 c_{j}-r$. Já que as modificações $n_{2} n_{1}$ e $n_{3} n_{1}$ transformam a relação $R_{3} R_{2} R_{1} R_{0}=1$ (colocamos a marca $c_{0}$ em $p_{3}$ ) na relação $R_{3} R_{2}^{R_{2} R_{1}} R_{1}^{R_{2}} R_{0}=1$ e $R_{3} R_{2}^{R_{1} R_{3}} R_{1} R_{0}^{R_{3} R_{1}}=1$, respectivamente, assumindo $c_{3}:=0 \in \mathbb{R}$, podemos ver que $n_{2} n_{1}:\left(c_{1}, c_{2}\right) \mapsto\left(2 c_{2}-c_{1}, 2 c_{2}-\left(2 c_{1}-c_{2}\right)\right)=\left(-c_{1}+2 c_{2},-2 c_{1}+\right.$ $\left.3 c_{2}\right)$ e $n_{3} n_{1}:\left(c_{1}, c_{2}\right) \mapsto\left(c_{1}, 2 c_{1}+c_{2}\right)$. Em outras palavras, nós identificamos $S$ com $\mathbb{R}^{2} / \pm 1$ onde $n_{2} n_{1}$ age como $\left[\begin{array}{ll}-1 & 2 \\ -2 & 3\end{array}\right]$ e $n_{3} n_{1}$, as $\left[\begin{array}{ll}1 & 0 \\ 2 & 1\end{array}\right]$. Resta observar que $\left[\begin{array}{ll}1 & 0 \\ 1 & 1\end{array}\right]\left[\begin{array}{ll}1 & 2 \\ 0 & 1\end{array}\right]\left[\begin{array}{cc}1 & 0 \\ -1 & 1\end{array}\right]=\left[\begin{array}{ll}-1 & 2 \\ -2 & 3\end{array}\right] \mathrm{e}$ $\left[\begin{array}{ll}1 & 0 \\ 1 & 1\end{array}\right]\left[\begin{array}{ll}1 & 0 \\ 2 & 1\end{array}\right]\left[\begin{array}{cc}1 & 0 \\ -1 & 1\end{array}\right]=\left[\begin{array}{ll}1 & 0 \\ 2 & 1\end{array}\right]$.

\subsection{Esferas hiperbólicas com $n+1$ pontos cônicos e $2 n$ - gonos convexos}

Dados $a_{0}, a_{1}, \ldots, a_{n} \in\left[\frac{\pi}{2}, \pi\left[\right.\right.$ tais que $\sum_{j} a_{j}>2 \pi$, nós consideramos o espaço $C\left(a_{0}, a_{1}, \ldots, a_{n}\right)$ de 2-esferas hiperbólicas $\Sigma$ com singularidades cônicas marcadas $c_{0}, c_{1}, \ldots, c_{n}$ tais que a curvatura em $c_{j}$ vale $2 a_{j}$ para todo $j$; as 2-esferas são consideradas a menos de orientação- e isometrias preservando marcas. 
Seja $\Sigma \in C$. Escolhemos segmentos geodésicos $\left[c_{0}, c_{j}\right] \subset \Sigma$ cujas intersecções dois a dois são apenas $c_{0}$ (digamos, escolhendo os mais curtos) e cortamos $\Sigma$ ao longo desses segmentos. Nós ganhamos um $2 n$-gono hiperbólico com vértices consecutivos $c_{1}, p_{1}, \ldots, c_{n}, p_{n}$ tal que o ângulo interior em $c_{j}$ é $2 \pi-2 a_{j} \leqslant \pi$ para todo $1 \leqslant j \leqslant n$ e os ângulos interiores nos $p_{j}$ 's somam $2 \pi-2 a_{0} \leqslant \pi$. Segue que $P$ é uma região tipo estrela, logo, é mergulhável pelo Fato 11 no plano hiperbólico como um 2-gono convexo. Aplicando a $P$ a modificação $m_{j, l}$ (veja Definição 3), obtemos outro $2 n$-gono convexo $P m_{j, l}$ tal que a esfera $\Sigma$ é colada de $P m_{j, l}$.

Nesta seção, mostramos que $C\left(a_{0}, a_{1}, \ldots, a_{n}\right)=S_{0}\left(a_{0}, a_{1}, \ldots, a_{n}\right) / M_{n}$, onde $M_{n}$ é o grupo gerado pelas modificações $m_{j, l}$ e $S\left(a_{0}, a_{1}, \ldots, a_{n}\right) \supset S_{0}\left(a_{0}, a_{1}, \ldots, a_{n}\right)$ é a união das $(n-1)$ ! componentes formadas por todos $2 n$-gonos convexos em $S\left(a_{0}, a_{1}, \ldots, a_{n}\right)$. As componentes em questão correspondem a tipos de $2 n$-gonos (veja Subseção 2.1.4 para a definição de tipo). As outras componentes de $S\left(a_{0}, a_{1}, \ldots, a_{n}\right)$ são formadas pelos $2 n$-gonos que são não-simples como curvas fechadas. É fácil ver que tais $2 n$-gonos também podem ser caracterizados como aqueles que 'limitam' uma área diferente de $2 \sum_{j} a_{j}-(n+4) \pi$ (veja [Ana1] para a definição da área 'limitada' por uma curva fechada não-simples).

Teorema 2. Sejam $a_{0}, a_{1}, \ldots, a_{n} \in\left[\frac{\pi}{2}, \pi\left[\right.\right.$ tais que $\sum_{j} a_{j}>2 \pi$. Dados $2 n$-gonos convexos $P, P^{\prime} \in$ $S_{0}\left(a_{0}, a_{1}, \ldots, a_{n}\right)$ tais que as correspondentes 2-esferas $\Sigma, \Sigma^{\prime}$ são isométricas com orientação e marcas preservadas, existe $m \in M_{n}$ tal que $P^{\prime}=P m$.

Observação 2. Tomamos um $2 n$-gono $P \in S_{0}\left(a_{0}, a_{1}, \ldots, a_{n}\right)$ com vértices consecutivos $c_{1}, p_{1}, \ldots, c_{n}, p_{n}$. Sem perda de generalidade, assumimos que os ângulos interiores a $P$ nos $p_{j}$ 's somam $2 \pi-2 a_{0} \mathrm{e}$ os ângulos interiores de $P \operatorname{nos} c_{j}$ são $2 \pi-2 a_{j}$ para todo $1 \leqslant j \leqslant n$. Denote por $D$ o disco fechado limitado no plano hiperbólico por $P$, seja $\Sigma$ a 2-esfera colada de $P$, e sejam $p, c_{1}, \ldots, c_{n} \in \Sigma$ respectivamente os pontos cônicos de curvaturas $2 a_{0}, 2 a_{1}, \ldots, 2 a_{n}$. Os lados de $P$ (depois da colagem) correspondem a segmentos geodésicos simples $s_{j} \subset \Sigma$ ligando respectivamente $p$ e $c_{j}$, $1 \leqslant j \leqslant n$, com intersecção dois a dois igual a $p$.

Qualquer $2 n$-gono $P^{\prime} \in S_{0}\left(a_{0}, a_{1}, \ldots, a_{n}\right)$ que produz o mesmo $\Sigma$ depois da colagem é portanto nada mais que segmentos geodésicos simples $s_{j}^{\prime} \subset \Sigma$ ligando respectivamente $p$ e $c_{j}$, $1 \leqslant j \leqslant n$, e $p$ é a intersecção dois a dois destes. Alguns dos $s_{j}$ 's podem coincidir com alguns $\operatorname{dos} s_{j}^{\prime}$. Denotemos por $0 \leqslant u \leqslant n$ o número de não-coincidentes. É claro que, $P^{\prime}=P$ se $u=0$.

Em termos do disco fechado $D$, um segmento arbitrário $s_{j}^{\prime}$ é dado por finitos segmentos geodésicos disjuntos $\left[q_{0}, q_{1}^{\prime}\right],\left[q_{1}, q_{2}^{\prime}\right], \ldots,\left[q_{v-1}, q_{v}^{\prime}\right] \subset D$, onde $1 \leqslant v, q_{0}=p_{k}$ para algum $1 \leqslant$ $k \leqslant n, q_{v}^{\prime}=c_{j}$, o interior de $\left[q_{l}, q_{l+1}^{\prime}\right]$ vive no interior de $D$ para todo $0 \leqslant l \leqslant v-1$ a menos quando $v=1$ e $k=j-1, j$, e, para todo $1 \leqslant l \leqslant v-1$, os pontos $q_{l}^{\prime} \neq q_{l}$ estão no interior de diferentes lados de $P$ (colados em $\Sigma$ ), simétricos em relação ao vértice comum $c_{w_{l}}, 1 \leqslant w_{l} \leqslant n$, i.e., $\operatorname{dist}\left(q_{l}^{\prime}, c_{w_{l}}\right)=\operatorname{dist}\left(c_{w_{l}}, q_{l}\right)$. Claramente, $s_{j}^{\prime}=s_{j}$ se $v=1$ e $k=j-1, j$.

Demonstração do Teorema 2 Trabalharemos no cenário da observação 2 e iremos proceder por indução em $u$ e então, por indução no número de intersecções dos segmentos 
não-coincidentes vindos de $P^{\prime}$ com aqueles vindos de $P$. Como não contamos como intersecções os pontos $p, c_{1}, \ldots, c_{n}$, as intersecções em questão são representadas por pontos no interior de $D$ ou no interior dos lados de $P$.

$\mathrm{O}$ caso $u=0$ foi feito na observação 2. Portanto, assumimos $u \geqslant 1$. Isso significa que existe $1 \leqslant j \leqslant n$ tal que $s_{j}^{\prime} \neq s_{j}$. Portanto, obtemos $v \geqslant 1$ segmentos geodésicos disjuntos como na observação 2 .

Afirmamos que os $2 n$-gonos $P m_{k, w}$ e $P^{\prime}$ satisfazem a hipótese de indução, onde $w:=w_{1}$ se $v>1$ e $w:=j$ se $v=1$.

Suponha que $v=1$. Como $s_{j}^{\prime} \neq s_{j}$, concluímos que $k \neq j-1, j$ (veja observação 2) e que $m_{k, j}$ é de fato aplicável. Ainda mais, quando passamos de $P$ para $P m_{k, j}$, o segmento $s_{j} \subset \Sigma$ é substituído pelo segmento $s_{j}^{\prime} \subset \Sigma$ e o resto dos segmentos vindos de $P$ se mantêm os mesmos. Noutras palavras, $u$ decresce.

Suponha que $v>1$. Então $k \neq w_{1}-1, w_{1}$ pois o interior de $\left[q_{0}, q_{1}^{\prime}\right]=\left[p_{k}, q_{1}^{\prime}\right]$ vive no interior de $D$. Então, $m_{k, w}$ é aplicável. Quando passamos de $P$ para $P m_{k, w}$, nós simplesmente trocamos $s_{w}$ pelo segmento $s$ representado por $\left[c_{w}, q_{0}\right]$. Então, $u$ não pode crescer e de fato se mantém o mesmo. Toda intersecção com $s$ pode ser vista como uma intersecção com $\left[c_{w}, q_{0}\right]$ vivendo no interior de $D$. Olhando mais de perto no triângulo com vértices $q_{0}, q_{1}^{\prime}, c_{w}$, nós entendemos que toda intersecção de algum $s_{l}^{\prime} \operatorname{com} s$ pode ser vista como uma intersecção de $s^{\prime} \subset D$, um dos segumentos relacionado a $s_{l}^{\prime}$ (como em 5.2), com $\left[c_{w}, q_{0}\right]$. O último induz uma intersecção de $s^{\prime} \operatorname{com}\left[q_{1}^{\prime}, c_{w}\right]$ porque $s^{\prime}$ e $\left[q_{0}, q_{1}^{\prime}\right]$ são disjuntas: elas são relacionadas a diferentes segmentos vindo de $P^{\prime}$. Resta observar que as intersecções correspondendo a $q_{1}^{\prime}$ desaparecem quando passamos de $P$ para $P m_{k, w}$. 


\title{
HYPERBOLIC 2-SPHERES WITH CONE SINGULARITIES
}

\author{
Sasha Anan'in, Carlos H. Grossi, Jaejeong Lee, João dos Reis JR.
}

\begin{abstract}
We study the space $C\left(a_{0}, a_{1}, \ldots, a_{n}\right)$ of hyperbolic 2-spheres with cone points of prescribed apex curvatures $\left.2 a_{0}, 2 a_{1}, \ldots, 2 a_{n} \in\right] 0,2 \pi[$ and some related spaces. For $n=3$, we get a detailed description of such spaces. The euclidean 2 -spheres were considered by W. P. Thurston: for $n=4$, the corresponding spaces provide the famous 7 examples of nonarithmetic compact holomorphic 2-ball quotients previously constructed by Deligne-Mostow.
\end{abstract}

To Misha Kapovich, a geometric geometer

\section{Introduction}

In the seminal work [Thu], W. P. Thurston studied the space of flat 2-spheres with cone points of prescribed apex curvatures. Such a space possesses a structure of complex hyperbolic manifold, though, is not in general complete. Its completion has a natural structure of complex hyperbolic cone manifold which turns out to be an orbifold exactly when satisfies a certain simple orbifold condition on the prescribed apex curvatures. In this way, Thurston reobtained the celebrated 7 nonarithmetic compact holomorphic 2-ball quotients (for short, nonarithmetic quotients) that were constructed by DeligneMostow [DM1], [DM2] about 30-35 years ago. As we are not going to distinguish a quotient $Q$ from a unramified finite cover of $Q$, we assume $Q$ to be a manifold. Recently, 4 more nonarithmetic quotients were constructed in [DPP]. The latter 4 are quite similar to the former 7 . Each of 11 contains at least four $\mathbb{C}$-fuchsian curves, i.e., smooth holomorphic totally geodesic compact curves.

In turn, the arithmetic quotients are known to be of 2 types [McR]: the first type and the second type which is related to division algebras. Taking into account the Mostow-Prasad rigidity of quotients, there are countably many arithmetic quotients of either type. The types can be distinguished by the presence of a $\mathbb{C}$-fuchsian curve: a quotient of the first type possesses such a curve, whereas that of the second one does not. Using $\mathbb{C}$-fuchsian curves, we define the type of an arbitrary quotient with the same criterion and arrive at the following questions.

1.1. Problem. Is there any nonarithmetic quotient of the second type? Do there exist infinitely many nonarithmetic quotients of the first/second type?

1.2. Outline. In this paper, we develop a few tools allowing to study the space $C\left(a_{0}, a_{1}, \ldots, a_{n}\right)$ of 2 -spheres with cone points of prescribed apex curvatures $2 a_{0}, 2 a_{1}, \ldots, 2 a_{n}$, where the set of the other points is endowed with the spherical geometry of curvature $\sigma:=1$ or with the hyperbolic geometry of curvature $\sigma:=-1$. Hopefully, $C\left(a_{0}, a_{1}, \ldots, a_{n}\right)$ or related spaces can shed some light on the above problem.

1.2.1. Toy example. In order to clarify the general picture, we first glance at the toy case of 4 cone points of the fixed apex curvatures $2 a, \pi, \pi, \pi$, where $a \in] 0, \frac{\pi}{2}[$ when $\sigma=1$ and $a \in] \frac{\pi}{2}, \pi[$ when

2000 Mathematics Subject Classification. 30F60 (22E40, 20H10).

Key words and phrases. Nonarithmetic/arithmetic compact holomorphic 2-ball quotients, spaces of spherical/hyperbolic structure with prescribed cone singularities on a 2 -sphere, discrete actions, braid groups. 
$\sigma=-1$. In this particular situation, each spherical or hyperbolic 2 -sphere can be cut into a solid triangle (closed disc) bounded by a triangle and living in the model space, i.e., in the round sphere or in the hyperbolic plane. Indeed, we simply join the cone point $p$ of apex curvature $2 a$ with the other 3 cone points by means of simple geodesic segments so that the segments intersect only in $p$ (say, taking the shortest ones) and then cut the 2 -sphere along the segments. The point $p$ turns into the vertices of the triangle and the other 3 cone points, into the middle points of the sides of the triangle. By splitting the solid triangle along a median and then gluing the corresponding half-sides, we modify the original triangle with respect to the median. As it happens, the 2 -spheres corresponding to triangles $t_{1}$ and $t_{2}$ are isometric (orientation preserved) iff $t_{1}$ and $t_{2}$ differ by finitely many such modifications. This allows us to describe the space $C\left(a, \frac{\pi}{2}, \frac{\pi}{2}, \frac{\pi}{2}\right)$ as the quotient of a topological disc $S$, the space of counterclockwise oriented triangles of the fixed area $\sigma(\pi-2 a)$, by the group $M_{3}$ generated by 3 involutions which are nothing but the modifications acting on $S$. In a certain sense, this construction is reminiscent of [WaW].

1.2.2. General case. Denote by $G$ the Lie group of orientation-preserving (= holomorphic) isometries of the model space. So, $G:=\mathrm{PU}(2)$ when $\sigma=1$ and $G:=\mathrm{PU}(1,1)$ when $\sigma=-1$. Let $c_{0}, c_{1}, \ldots, c_{n}$ stand for the cone points of some 2 -sphere $\Sigma \in C\left(a_{0}, a_{1}, \ldots, a_{n}\right)$. Then we get a holonomy representation $\varrho: F_{n} \rightarrow G$, where $F_{n}:=\pi_{1}\left(\Sigma \backslash\left\{c_{0}, c_{1}, \ldots, c_{n}\right\}\right)$ is the free group of rank $n$. In this way, we obtain a well-defined holonomy map $h: C\left(a_{0}, a_{1}, \ldots, a_{n}\right) \rightarrow \operatorname{REP}\left(F_{n}, G\right) / G, \Sigma \mapsto[\varrho]$, to the space of representations $\operatorname{ReP}\left(F_{n}, G\right)$ considered modulo conjugation by $G$.

As in 1.2.1, we can join $c_{0}$ with $c_{1}, \ldots, c_{n}$ by means of $n$ simple geodesic segments so that the segments intersect only in $c_{0}$ (say, taking the shortest ones) and then cut $\Sigma$ along the segments thus getting a $2 n$ gon $P$ that bounds a topological closed disc not necessarily embeddable into the model space. Labeling the odd vertices of $P$ that originate from $c_{1}, \ldots, c_{n}$ with the same symbols and the even ones, that originate from $c_{0}$, with $p_{1}, \ldots, p_{n}$, we see that the interior angles of $P$ at the $p_{j}$ 's sum to $2 \pi-2 a_{0}$, that the interior angle of $P$ at $c_{j}$ equals $2 \pi-2 a_{j}$, and that the sides of $P$ adjacent at $c_{j}$ have equal length for all $j=1, \ldots, n$. In some sense, $P$ bounds a fundamental domain for $\Sigma$.

A choice of the above segments distinguishes some generators $r_{0}, r_{1}, \ldots, r_{n}$ of $F_{n}$ such that $F_{n}=$ $\left\langle r_{0}, r_{1}, \ldots, r_{n} \mid r_{n} \ldots r_{1} r_{0}=1\right\rangle$. A suitable braid group $\bar{M}_{n}$ (see Definition 4.2) acts on $F_{n}$ and preserves the set of conjugacy classes of the $r_{j}$ 's. Another choice of the segments is given at the level of representations by an element $m \in \bar{M}_{n}$ that transforms $[\varrho]$ into $[\varrho \circ m]$. (In general, such elements $m$ do not form a subgroup.) Hence, the image of the holonomy map $h$ lives in a sort of relative character variety $R\left(a_{0}, a_{1}, \ldots, a_{n}\right) \subset \operatorname{REP}\left(F_{n}, G\right) / G$ formed by all the representations $[\varrho]$ such that the conjugacy classes of $\varrho r_{0}, \varrho r_{1}, \ldots, \varrho r_{n}$ are the counterclockwise rotations in the model space by the angles $2 a_{0}, 2 a_{1}, \ldots, 2 a_{n}$, listed perhaps in different order. In alternative words, $R\left(a_{0}, a_{1}, \ldots, a_{n}\right)$ is the space of relations between rotations in prescribed conjugacy classes (the order of the classes is not fixed).

1.2.3. Convex hyperbolic $2 n$-gons. Let us look more closely at the case of $\sigma=-1$ with $a_{0}, a_{1}, \ldots, a_{n} \in\left[\frac{\pi}{2}, \pi\left[\right.\right.$ such that $\sum_{j} a_{j}>2 \pi$. Here, any $2 n$-gon $P$ is convex, i.e., its interior angles are all in $] 0, \pi]$, hence, the corresponding closed disc is embeddable into the model space. In this case, the space $C\left(a_{0}, a_{1}, \ldots, a_{n}\right)$ of hyperbolic 2 -spheres with cone points of apex curvatures $2 a_{0}, 2 a_{1}, \ldots, 2 a_{n}$ can be described as the quotient of a component of $R\left(a_{0}, a_{1}, \ldots, a_{n}\right)$ by the action of $\bar{M}_{n}$ (see Section 5 and Theorem 5.1; see also Section 1.3).

1.2.4. Geometry on $C\left(a_{0}, a_{1}, \ldots, a_{n}\right)$ and on the related spaces. Since we hope to endow $C\left(a_{0}, a_{1}, \ldots, a_{n}\right)$ with a structure of complex hyperbolic orbifold, it is natural to conjecture that such a geometry descents from a component $C$ of $R\left(a_{0}, a_{1}, \ldots, a_{n}\right)$ and that $\bar{M}_{n}$ acts discretely on $C$ by isometries. (There is a more subtle approach to constructing spaces with geometry in a similar vein, but let us stick first to a simplest variant.)

Actually, even in the flat case considered by Thurston, there may exist no preferred hyperbolic geometry on $C$. (Well, in the flat case, one can always choose the structure related to the oriented area 
function; it does provide a complex hyperbolic structure.) The choice of a 'good' hyperbolic structure on $C$ is the principal challenge in our project, and the first step is to describe the components of $R\left(a_{0}, a_{1}, \ldots, a_{n}\right)$ where $\bar{M}_{n}$ acts discretely.

One can establish the lack of geometry of constant curvature on a component of $R\left(a_{0}, a_{1}, a_{2}, a_{3}\right)$, compatible with the action of $\bar{M}_{3}$, if this action is not discrete.

1.2.5. Brief sketch of the exposition. Every representation $[\varrho] \in R\left(a_{0}, a_{1}, \ldots, a_{n}\right)$ can be interpreted as a labeled $2 n$-gon, a closed piecewise geodesic oriented path, whose consecutive vertices $c_{1}, p_{1}, \ldots, c_{n}, p_{n}:=c_{0}$ are given by $p_{j}:=R_{j} p_{j-1}$ for all $1 \leqslant j \leqslant n$ (the indices are modulo $n$ ), where $c_{j}$ stands for the fixed point of the rotation $R_{j}:=\varrho r_{j}, 0 \leqslant j \leqslant n$, and, without loss of generality, $R_{0}$ is a counterclockwise rotation by $2 a_{0}$.

It is convenient to deal with the $2 n$-gons with a forgotten label $c_{0}$. In other words, we consider the quotient $S\left(a_{0}, a_{1}, \ldots, a_{n}\right)$ of $R\left(a_{0}, a_{1}, \ldots, a_{n}\right)$ by a suitable order $n$ cyclic subgroup in $\bar{M}_{n}$. Thus, we are allowed to place the fixed point of the rotation $R_{0}$ at any vertex $p_{j}$. In terms of relations, the generator of the cyclic group transforms the relation $R_{n} \ldots R_{1} R_{0}=1$ into the relation $R_{1} R_{n} \ldots\left(R_{1} R_{0} R_{1}^{-1}\right)=1$. A certain braid group $M_{n}$ (see Definition 4.3) generated by simple modifications (surgeries) of the $2 n$ gons acts on $S\left(a_{0}, a_{1}, \ldots, a_{n}\right)$ so that the map $R\left(a_{0}, a_{1}, \ldots, a_{n}\right) \rightarrow S\left(a_{0}, a_{1}, \ldots, a_{n}\right)$ induces a bijection between the $\bar{M}_{n}$-orbits and the $M_{n}$-orbits. Clearly, the discreteness of $\bar{M}_{n}$ is equivalent to that of $M_{n}$.

Next step is to describe the components of $S\left(a_{0}, a_{1}, \ldots, a_{n}\right)$. The cyclic order of $a_{1}, \ldots, a_{n}$ in $P$ defines the type of a $2 n$-gon $P$ and $M_{n}$ acts transitively on the types. Thus, we need to describe the components of a given type and to find those components where $M$, the index $(n-1)$ ! subgroup of the type-preserving elements of $M_{n}$, acts discretely.

We proceed by induction on $n$. Without loss of generality, we assume $R_{0}$ and $R_{n}$ to be counterclockwise rotations by $2 a_{0}$ and $2 a_{n}$. The conjugacy class of $R_{0} R_{n}$ is given exactly by the distance $d_{n}$ between $c_{n}$ and $p_{n}$. Therefore, by induction, we know the topology of a fibre of the map $d_{n}: S\left(a_{0}, a_{1}, \ldots, a_{n}\right) \rightarrow\left[0, \infty\left[\right.\right.$. Indeed, if $R_{0} R_{n}$ is a counterclockwise rotation by $\left.2 a, a \in\right] 0, \pi[$, then the fibre in question is $S\left(a, a_{1}, \ldots, a_{n-1}\right) \times C_{R_{0} R_{n}}$, where $C_{R_{0} R_{n}}$ stands for the centralizer of $R_{0} R_{n}$ in $G$, topologically a circle. If $R_{0} R_{n}=1$ (hence, $d_{n}=0$ ), then the fibre equals $S\left(a_{1}, \ldots, a_{n-1}\right)$. If $R_{0} R_{n}$ is a parabolic or hyperbolic isometry, then the fibre is $S\left(a^{*}, a_{1}, \ldots, a_{n-1}\right) \times C_{R_{0} R_{n}}$, where $a^{*}$ stands for the conjugacy class of $R_{0} R_{n}$ and $C_{R_{0} R_{n}}$ is topologically a line. (This means that, for the sake of induction, we need to consider the relations between isometries in prescribed conjugacy classes, not necessarily elliptic ones. We do not do so in this particular paper because we study here mostly the case of $n=3$ and $\sigma=-1$.) Gathering information about the topology of the fibres of $d_{n}$, including that at the singular points of $d_{n}$, we arrive at the description of the components and their topology (see Proposition 4.5.2).

Also, in the case $n=3$ and $\sigma=-1$, we visualize 3 curves $A_{1}, A_{2}, A_{3}$ that are respectively fixed point sets of the involutions $n_{1}, n_{2}, n_{3}$ that generate the group $M_{3}$ (here we identify the components of both types). We show that $M_{3}$ does not act discretely on a component if the component is not smooth (see Remark 4.6.4) or if a couple of the above curves intersects in the component. Finally, we describe all components where $M_{3}$ acts discretely (see Theorem 4.6.3) : for any $\left.a_{0}, a_{1}, a_{2}, a_{3} \in\right] 0, \pi[$, unless $a_{0}=a_{1}=a_{2}=a_{3}=\frac{\pi}{2}$ (the case of nonsmooth $S\left(a_{0}, a_{1}, a_{2}, a_{3}\right)$ ), there exists a component (of a given type) where $M_{3}$ acts discretely iff $a_{j}+a_{k} \leqslant \pi$ for all $j \neq k$ or $\pi \leqslant a_{j}+a_{k}$ for all $j \neq k$, and such a component is unique. Taking an ideal triangle on the hyperbolic plane and the group generated by the reflections in its sides $A_{1}, A_{2}, A_{3}$, we get an adequate topological picture of the action of $M_{3}$ on a component in question.

It is worthwhile mentioning that any component of $S\left(a_{0}, a_{1}, a_{2}, a_{3}\right)$ is a surface in $\mathbb{R}^{3}$ given by one of the equations (3.5) or (3.7).

1.3. Related works. Being back to the toy example 1.2 .1 , hence, taking in the equations $(3.5)$ and (3.7) $a_{1}=a_{2}=a_{3}:=\frac{\pi}{2}$, and changing the variables with respect to $x_{j}:= \pm 2 s\left(2 t_{j}-1\right)$, we arrive at the equation $x_{1}^{2}+x_{2}^{2}+x_{3}^{2}-x_{1} x_{2} x_{3}-2=t$, where $s:=\sin a_{0}, t:=-\operatorname{tr} R_{c, k}, k:=e^{a_{0} i}$, and $R_{c, k} \in \mathrm{SU}(1,1)$ 
(see the definition in the beginning of Section 2) represents the counterclockwise rotation by $2 a_{0}$ about $c$ in the hyperbolic plane. In other words, our group $M_{3}$ in this case is commensurable with the group $\Gamma$ from [Gol] in the case $|t|<2$. If we would admit in the toy example a parabolic or hyperbolic isometry in place of $R_{c, k}$, we could deal in fact with the general case studied by W. M. Goldman [Gol] (the parabolic isometry would correspond to the case studied by P. Waterman and S. Wolpert [WaW]).

The condition $\sum_{j=0}^{n} a_{j}>2 \pi$ (or $\sum_{j=0}^{n} a_{j}<2 \pi$ ) on the prescribed apex curvatures, necessary for the existence of a hyperbolic (respectively, spherical) 2-sphere $\Sigma$ with the indicated cone singularities, is clearly sufficient. Moreover, A. D. Alexandrov proved that, for any such $\Sigma$, there exists a unique convex compact polyhedron $P$ in the real hyperbolic 3 -space of curvature -1 (respectively, in the round 3 -sphere of curvature 1) whose boundary $\partial P$ is isometric to $\Sigma$ with respect to the inner metric on $\partial P$ (see [Ale, 3.6.4, p. 190] for the uniqueness and [Ale, 5.3.1, p. 261] for the existence).

G. Mondello and D. Panov [MPa] found necessary and almost sufficient conditions for the existence of a spherical 2-sphere with cone points of given cone angles, each of the angles is allowed to exceed $2 \pi$. The corresponding space can be quite useful when dealing with Problem 1.1. In the context of convex polyhedra in real hyperbolic 3-space, C. D. Hodgson and I. Rivin [HRi] described all spherical 2-spheres with all cone angles $>2 \pi$ and all closed geodesics of length $>2 \pi$.

Acknowledgements. We are very grateful to KIAS and to professor Sungwoon Kim for organizing 'Workshop on geometric structures, Hitchin components, and representation varieties' and supporting our collaboration (see http://home.kias.re.kr/MKG/h/WGS2015/). We also thank the referee for remarks that improved our exposition. The first three authors were supported by FAPESP, grant 2015/25809-2. The forth author was partially supported by FAPESP, grant 2014/26295-0.

\section{Preliminary remarks}

We are going to use the following settings and notation similar to those in [AGr].

Let $\sigma= \pm 1$. In what follows, $V$ denotes a 2-dimensional $\mathbb{C}$-linear space equipped with a hermitian form $\langle-,-\rangle$ of signature $\left(1+\frac{\sigma+1}{2}, \frac{\sigma-1}{2}\right)$. Denote B $V:=\left\{p \in \mathbb{P}_{\mathbb{C}} V \mid\langle p, p\rangle>0\right\}$. Then $\mathrm{B} V$ is the model space of curvature $\sigma$, a round sphere if $\sigma=1$ and a hyperbolic disc if $\sigma=-1$. In fact, when $\sigma=-1$, the Riemann-Poincaré sphere $\mathbb{P}_{\mathbb{C}} V$ is glued from two hyperbolic discs $\mathrm{B} V$ and $\mathrm{B}^{\prime} V:=\left\{p \in \mathbb{P}_{\mathbb{C}} V \mid\right.$ $\langle p, p\rangle\langle 0\}$ along the absolute $\partial \mathrm{B} V:=\left\{p \in \mathbb{P}_{\mathbb{C}} V \mid\langle p, p\rangle=0\right\}$.

The distance $\operatorname{dist}\left(p_{1}, p_{2}\right)$ between points $p_{1}, p_{2} \in \mathrm{B} V$ is given by the formulae $\cos ^{2} \frac{\operatorname{dist}\left(p_{1}, p_{2}\right)}{2}=$ $\operatorname{ta}\left(p_{1}, p_{2}\right)$ for $\sigma=1$ and $\cosh ^{2} \frac{\operatorname{dist}\left(p_{1}, p_{2}\right)}{2}=\operatorname{ta}\left(p_{1}, p_{2}\right)$ for $\sigma=-1$. So, the distance is a monotonic function of the tance defined as $\operatorname{ta}\left(p_{1}, p_{2}\right):=\frac{\left\langle p_{1}, p_{2}\right\rangle\left\langle p_{2}, p_{1}\right\rangle}{\left\langle p_{1}, p_{1}\right\rangle\left\langle p_{2}, p_{2}\right\rangle}$.

For $z \in \mathbb{C} \backslash 0$, we denote by $\arg z$ and $\operatorname{Arg} z$ the argument functions taking values in $]-\pi, \pi]$ and in $[0,2 \pi[$, respectively.

2.1. Remark [AGr, Example 6.1]. The oriented area of a triangle with pairwise nonorthogonal vertices $p_{1}, p_{2}, p_{3} \in \mathrm{B} V$ equals area $\left(p_{1}, p_{2}, p_{3}\right)=-2 \sigma \arg \left(g_{12} g_{23} g_{31}\right)$, where $\left[g_{j k}\right]$ stands for the Gram matrix of the $p_{j}$ 's. (If $\sigma=-1$, it is possible to observe that arg takes values in ] $-\frac{\pi}{2}, \frac{\pi}{2}$ [ when calculating area by the above formula; if $\sigma=1$, then arg can take any value.) In particular, the triangle is counterclockwise oriented iff $-\sigma \operatorname{Im}\left(g_{12} g_{23} g_{31}\right)>0$.

For $c \in \mathrm{B} V$ and $k \in \mathbb{C}$ such that $|k|=1$, the rule $R_{c, k}: p \mapsto(\bar{k}-k) \frac{\langle p, c\rangle}{\langle c, c\rangle} c+k p$ defines a $\mathbb{C}$-linear $\operatorname{map} R_{c, k}: V \rightarrow V$.

2.2. Remark. Let $c, p \in \mathrm{B} V$ and let $k, k^{\prime} \in \mathbb{C}$ be such that $|k|=\left|k^{\prime}\right|=1$. Then $R_{c, k}$ provides a rotation in $\mathrm{B} V$. More precisely, $R_{c, k}$ is the rotation by $\operatorname{Arg} k^{2}$ in the counterclockwise sense about $c$. Moreover, $R_{c, k} \in \mathrm{SU} V, R_{c,-k}=-R_{c, k}, R_{c, k} R_{c, k^{\prime}}=R_{c, k k^{\prime}},\left\langle c, R_{c, k} p\right\rangle=k\langle c, p\rangle$, and $\left\langle R_{c, k} p, p\right\rangle=$ $(\operatorname{ta}(p, c)(\bar{k}-k)+k)\langle p, p\rangle$. If $\sigma=1$ and $\left\langle c, c^{\prime}\right\rangle=0$, then $R_{c, k} R_{c^{\prime}, k}=1$. 
Proof. Let $c^{\prime} \in \mathbb{P}_{\mathbb{C}} V$ be the point orthogonal to $c$ and take representatives such that $\langle c, c\rangle=1$ and $\left\langle c^{\prime}, c^{\prime}\right\rangle=\sigma$. Every point $x \in \mathrm{B} V$ can be written in the form $x=c+\eta c^{\prime}$ for some unique $\eta \in \mathbb{C}$. When $\sigma=-1$, we have $|\eta|<1$ and this corresponds to identifying B $V$ with the unitary disc in $\mathbb{C}$ centred at the origin; when $\sigma=1$, we identified $\mathbb{P}_{\mathbb{C}} V \backslash c^{\prime}$ with $\mathbb{C}$ (in both cases, the point $c$ is mapped to the origin). Under this identification, $R_{c, k}$ sends the point $x$ to $c+k^{2} \eta c^{\prime}$, i.e., it is nothing but multiplication by the unitary complex number $k^{2}$. In the orthonormal basis $c, c^{\prime}$, the map $R_{c, k}$ has the form $\left[\begin{array}{cc}\bar{k} & 0 \\ 0 & k\end{array}\right]$, so it belongs to SUV. The equalities $R_{c,-k}=-R_{c, k}, R_{c, k} R_{c, k^{\prime}}=R_{c, k k^{\prime}},\left\langle c, R_{c, k} p\right\rangle=k\langle c, p\rangle$, and $\left\langle R_{c, k} p, p\right\rangle=(\operatorname{ta}(p, c)(\bar{k}-k)+k)\langle p, p\rangle$ follow from straightforward calculations. Finally, in order to show that $R_{c, k} R_{c^{\prime}, k}=1$, it suffices to note that $R_{c, k} R_{c^{\prime}, k} c=c$ and $R_{c, k} R_{c^{\prime}, k} c^{\prime}=c^{\prime}$.

Let $\gamma$ be a geodesic in $\mathrm{B} V$. We denote by $I_{\gamma}$ the (antiholomorphic) reflection in $\gamma$. Given a couple of geodesics $\gamma, \gamma^{\prime}$ intersecting at $c \in \mathrm{B} V$, denote by $\alpha_{c}\left(\gamma, \gamma^{\prime}\right) \in[0, \pi[$ the oriented angle at $c$ from $\gamma$ to $\gamma^{\prime}$ counted in the counterclockwise sense.

2.3. Remark. Let $1 \neq R \in \mathrm{PU} V$ be a nontrivial orientation-preserving isometry of $\mathrm{B} V$. Then there exist distinct geodesics $\gamma, \gamma^{\prime}$ such that $R=I_{\gamma^{\prime}} I_{\gamma}$ in $\mathrm{PU} V$.

The geodesics are ultraparallel, tangent, or intersecting iff $R$ is respectively hyperbolic, parabolic, or elliptic. The couple of geodesics is unique up to the action of the centralizer $C_{R}$ of $R$ in PU $V$ and the action of the centralizer is free in the hyperbolic and parabolic cases. In the elliptic case, the stabilizer of the couple is given by the reflection in a point of the intersection of the geodesics, i.e., by $R_{c, i}$, where $c$ is a point of the intersection. In this case, $I_{\gamma^{\prime}} I_{\gamma}=R_{c, k} \in \mathrm{PU} V$, where $k:=e^{i \alpha_{c}\left(\gamma, \gamma^{\prime}\right)}$.

Topologically, $C_{R}$ is a circle if $R$ is elliptic and a line, if $R$ is hyperbolic or parabolic.

2.4. Remark. Let $\sigma=-1$. For any $\left.a_{1}, a_{2} \in\right] 0, \pi\left[\right.$ such that $a_{1}+a_{2} \neq \pi$, there exists unique $t_{0}\left(a_{1}, a_{2}\right)>1$ satisfying the following property. Given distinct points $q_{1}, q_{2} \in \mathrm{B} V$, denote by $\gamma_{1}, \gamma_{2}$ the geodesics such that $q_{j} \in \gamma_{j}, \alpha\left(\gamma, \gamma_{1}\right)=a_{1}$, and $\alpha\left(\gamma_{2}, \gamma\right)=a_{2}$, where $\gamma$ stands for the geodesic joining $q_{1}, q_{2}$. The geodesics $\gamma_{1}, \gamma_{2}$ intersect, are tangent, or are ultraparallel if $\operatorname{ta}\left(q_{1}, q_{2}\right)<t_{0}\left(a_{1}, a_{2}\right)$, $\operatorname{ta}\left(q_{1}, q_{2}\right)=t_{0}\left(a_{1}, a_{2}\right)$, or $\operatorname{ta}\left(q_{1}, q_{2}\right)>t_{0}\left(a_{1}, a_{2}\right)$, respectively. In the case $a_{1}+a_{2}=\pi$, the geodesics $\gamma_{1}, \gamma_{2}$ are always ultraparallel and we put $t_{0}\left(a_{1}, a_{2}\right):=1$ in this case.

2.5. Remark. Let $\gamma_{1}, \gamma_{2}$ be ultraparallel geodesics and let $\left.a_{1}, a_{2} \in\right] 0, \pi[$. Then there exists a unique geodesic $\gamma$, depending smoothly on $\gamma_{1}, \gamma_{2}$, such that $\alpha\left(\gamma_{1}, \gamma\right)=a_{1}$ and $\alpha\left(\gamma, \gamma_{2}\right)=a_{2}$.

Proof. Let $v_{1}, v_{1}^{\prime}$ and $v_{2}, v_{2}^{\prime}$ be vertices of $\gamma_{1}$ and of $\gamma_{2}$ such that the triangles $\left(v_{1}^{\prime}, v_{1}, v_{2}\right)$ and $\left(v_{1}^{\prime}, v_{2}, v_{2}^{\prime}\right)$ are both clockwise oriented. Then the angle from $\left[v_{1}^{\prime}, c\right]$ to $\left[c, v_{2}\right]$ grows monotonically from 0 to $\pi$ while $c \in \gamma_{1}$ runs from $v_{1}$ to $v_{1}^{\prime}$. At some $c \in \gamma_{1}$, this angle equals $a_{1}$. Similarly, there exists $c^{\prime} \in\left[c, v_{1}^{\prime}\right]$ such that the angle from $\left[v_{1}^{\prime}, c^{\prime}\right]$ to $\left[c^{\prime}, v_{2}^{\prime}\right]$ equals $a_{1}$. It follows that, for any $p \in\left[c, c^{\prime}\right]$, the geodesic $\gamma_{p}$ that passes through $p$ and such that $\alpha\left(\gamma_{1}, \gamma_{p}\right)=a_{1}$ intersects $\gamma_{2}$. For $p=c$, this intersection is $v_{2}$ and, for $p=c^{\prime}$, it is $v_{2}^{\prime}$. Hence, the angle $\alpha\left(\gamma_{p}, \gamma_{2}\right)$ varies from 0 to $\pi$. By continuity, we obtain the existence of the desired $\gamma$. If we have another $\gamma^{\prime}$, then $\gamma$ and $\gamma^{\prime}$ are ultraparallel. So, we get a simple quadrangle whose interior angles sum to $2 \pi$, a contradiction.

2.6. Remark. Let $\sigma=-1$ and $\left.a_{0}, a_{1}, a_{2}, a_{3} \in\right] 0, \pi\left[\right.$. Then, for any $t>t_{0}\left(a_{0}, a_{3}\right)$, there exist geometrically unique geodesics $\gamma, \gamma_{0}, \gamma_{1}, \gamma_{2}$ such that $\alpha\left(\gamma_{0}, \gamma_{1}\right)=a_{0}, \alpha\left(\gamma_{1}, \gamma\right)=a_{1}, \alpha\left(\gamma, \gamma_{2}\right)=a_{2}$, $\alpha\left(\gamma_{2}, \gamma_{0}\right)=a_{3}$, and $\operatorname{ta}\left(c_{3}, c_{0}\right)=t$ (hence, $\gamma_{1}, \gamma_{2}$ are ultraparallel), where $c_{0}$ and $c_{3}$ stand for the intersection points of $\gamma_{0}, \gamma_{1}$ and of $\gamma_{0}, \gamma_{2}$, respectively. Moreover, the four geodesics depend smoothly on $t>t_{0}\left(a_{0}, a_{3}\right)$.

Proof. By Remark 2.4, taking points $c_{0}, c_{3}$ on the tance ta $\left(c_{0}, c_{3}\right)=t$, we get ultraparallel geodesics $\gamma_{1}, \gamma_{2}$ such that $c_{0} \in \gamma_{1}$ with $\alpha\left(\gamma_{0}, \gamma_{1}\right)=a_{0}$ and $c_{3} \in \gamma_{2}$ with $\alpha\left(\gamma_{2}, \gamma_{0}\right)=a_{3}$, where $\gamma_{0}$ stands for the geodesic joining $c_{0}, c_{3}$. By Remark 2.5, there exists a unique geodesic $\gamma$ such that $\alpha\left(\gamma_{1}, \gamma\right)=a_{1}$ and $\alpha\left(\gamma, \gamma_{2}\right)=a_{2}$ 
2.7. Remark. Let $\sigma=-1$. Given distinct geodesics $\gamma_{1}, \gamma_{2}$ intersecting at some point $c \in \mathrm{B} V$, denote $a:=\alpha\left(\gamma_{2}, \gamma_{1}\right)$ and pick some $\left.a_{1}, a_{2} \in\right] 0, \pi\left[\right.$. Then there exists a couple of geodesics $\gamma, \gamma^{\prime}$ (subject to $\left.\gamma^{\prime}=R_{c, i} \gamma\right)$ such that $\alpha\left(\gamma_{1}, \gamma\right)=\alpha\left(\gamma_{1}, \gamma^{\prime}\right)=a_{1}$ and $\alpha\left(\gamma, \gamma_{2}\right)=\alpha\left(\gamma^{\prime}, \gamma_{2}\right)=a_{2}$ iff

- $a+a_{1}+a_{2} \leqslant \pi$, when the triangle $\left(c, c_{1}, c_{2}\right)$ is clockwise oriented or degenerate (i.e., $\left.c=c_{1}=c_{2}\right)$, or

- $2 \pi \leqslant a+a_{1}+a_{2}$, when the triangle $\left(c, c_{1}, c_{2}\right)$ is counterclockwise oriented or degenerate,

where $c_{j}$ stands for the intersection point of $\gamma$ and $\gamma_{j}$. Such geodesics $\gamma, \gamma^{\prime}$ are unique when exist and depend continuously on $\gamma_{1}, \gamma_{2}$, and $a$.

The condition $\gamma=\gamma^{\prime}$ is equivalent to $a+a_{1}+a_{2}=\pi$ or $a+a_{1}+a_{2}=2 \pi$; equivalently, this means that $c=c_{1}=c_{2}$.

2.8. Remark. Let $\sigma=-1$. Suppose that $\left.a_{0}, a_{1}, a_{2}, a_{3} \in\right] 0, \pi\left[\right.$ satisfy $a_{0}+a_{1}+a_{2}+a_{3}<\pi$ or $3 \pi<a_{0}+a_{1}+a_{2}+a_{3}$. Then there exist geodesics $\gamma, \gamma_{0}, \gamma_{1}, \gamma_{2}$ such that $\gamma_{1}, \gamma_{2}$ intersect, $\alpha\left(\gamma_{0}, \gamma_{1}\right)=a_{0}$, $\alpha\left(\gamma_{1}, \gamma\right)=a_{1}, \alpha\left(\gamma, \gamma_{2}\right)=a_{2}$, and $\alpha\left(\gamma_{2}, \gamma_{0}\right)=a_{3}$.

Proof. If $a_{0}+a_{1}+a_{2}+a_{3}<\pi$, then $0<a_{0}+a_{3}<\pi-a_{1}-a_{2}<\pi$. So, we can pick $\left.a \in\right] 0, \pi[$ such that $a_{0}+a_{3}<a<\pi-a_{1}-a_{2}$. Hence, there is a counterclockwise oriented triangle $\left(c, c_{0}, c_{3}\right)$ with the interior angles $\pi-a, a_{0}, a_{3}$, respectively. Denote by $\gamma_{0}, \gamma_{1}, \gamma_{2}$ the geodesics such that $c_{0}, c_{3} \in \gamma_{0}$, $c, c_{0} \in \gamma_{1}$, and $c_{3}, c \in \gamma_{2}$. As $a=\alpha\left(\gamma_{2}, \gamma_{1}\right)$ and $a+a_{1}+a_{2}<\pi$, by Remark 2.7, there exists a geodesic $\gamma$ with $\alpha\left(\gamma_{1}, \gamma\right)=a_{1}$ and $\alpha\left(\gamma, \gamma_{2}\right)=a_{2}$.

If $3 \pi<a_{0}+a_{1}+a_{2}+a_{3}$, then $0<2 \pi-a_{1}-a_{2}<a_{0}+a_{3}-\pi<\pi$. So, we can pick $\left.a \in\right] 0, \pi[$ such that $2 \pi-a_{1}-a_{2}<a<a_{0}+a_{3}-\pi$. Hence, there is a clockwise oriented triangle $\left(c, c_{0}, c_{3}\right)$ with the interior angles $a, \pi-a_{0}, \pi-a_{3}$, respectively. Denote by $\gamma_{0}, \gamma_{1}, \gamma_{2}$ the geodesics such that $c_{0}, c_{3} \in \gamma_{0}$, $c, c_{0} \in \gamma_{1}$, and $c_{3}, c \in \gamma_{2}$. As $a=\alpha\left(\gamma_{2}, \gamma_{1}\right)$ and $2 \pi<a+a_{1}+a_{2}$, by Remark 2.7, there exists a geodesic $\gamma$ with $\alpha\left(\gamma_{1}, \gamma\right)=a_{1}$ and $\alpha\left(\gamma, \gamma_{2}\right)=a_{2}$.

2.9. Remark. Let $\gamma_{1}, \gamma_{2}$ be distinct tangent geodesics and let $\left.a_{1}, a_{2} \in\right] 0, \pi\left[\right.$. Denote by $v_{1}, v$ and by $v, v_{2}$ the vertices of $\gamma_{1}$ and of $\gamma_{2}$, respectively. Then there exists a geodesic $\gamma$ such that $\alpha\left(\gamma_{1}, \gamma\right)=a_{1}$ and $\alpha\left(\gamma, \gamma_{2}\right)=a_{2}$ iff $a_{1}+a_{2}<\pi$ and the triangle $\left(v_{1}, v, v_{2}\right)$ is counterclockwise oriented or $\pi<a_{1}+a_{2}$ and the triangle $\left(v_{1}, v, v_{2}\right)$ is clockwise oriented. Such a geodesic $\gamma$ is unique if exists.

In particular, any sufficiently small deformation of $\gamma_{1}, \gamma_{2}$ still allows a geodesic (a couple of geodesics) with the required angles.

2.10. Remark. Let $\sigma=-1$. Suppose that $\left.a_{0}, a_{1}, a_{2}, a_{3} \in\right] 0, \pi\left[\right.$ satisfy $a_{0}+a_{3}<\pi<a_{1}+a_{2}$ or $a_{1}+a_{2}<\pi<a_{0}+a_{3}$. Then there exist geodesics $\gamma, \gamma_{0}, \gamma_{1}, \gamma_{2}$ such that $\gamma_{1}, \gamma_{2}$ are tangent, $\alpha\left(\gamma_{0}, \gamma_{1}\right)=a_{0}$, $\alpha\left(\gamma_{1}, \gamma\right)=a_{1}, \alpha\left(\gamma, \gamma_{2}\right)=a_{2}$, and $\alpha\left(\gamma_{2}, \gamma_{0}\right)=a_{3}$.

Proof. Pick a couple of distinct tangent geodesics $\gamma_{1}, \gamma_{2}$ such that, in terms of Remark 2.9, the triangle $\left(v_{1}, v, v_{2}\right)$ is clockwise oriented if $a_{0}+a_{3}<\pi$ and counterclockwise oriented if $\pi<a_{0}+a_{3}$. By Remark 2.9, there exists a unique geodesic $\gamma_{0}$ with $\alpha\left(\gamma_{2}, \gamma_{0}\right)=a_{3}$ and $\alpha\left(\gamma_{0}, \gamma_{1}\right)=a_{0}$. Again by Remark 2.9, there exists a unique geodesic $\gamma$ with $\alpha\left(\gamma_{1}, \gamma\right)=a_{1}$ and $\alpha\left(\gamma, \gamma_{2}\right)=a_{2}$ because $a_{0}+a_{3}<\pi$ implies $\pi<a_{1}+a_{2}$ and $\pi<a_{0}+a_{3}$ implies $a_{1}+a_{2}<\pi$.

2.11. Remark. Let $\Sigma$ be a hyperbolic 2 -sphere with cone points $(\sigma=-1)$ and let $R \subset \Sigma$ be a connected and simply connected region containing no cone points. Then there is a locally isometric immersion from $R$ into $\mathrm{B} V$. If $R$ is a star-like region, then the immersion is an embedding.

\section{Technical lemmas}

3.1. Lemma. Let $k_{0}, k_{1}, k_{2}, k_{3} \in \mathbb{C}$ with $\left|k_{j}\right|=1$ be fixed. Pick some $c_{j} \in \mathrm{B} V, 0 \leqslant j \leqslant 3$, and denote $t_{1}:=\operatorname{ta}\left(c_{0}, c_{1}\right), t_{2}:=\operatorname{ta}\left(R_{c_{1}, k_{1}} c_{0}, c_{2}\right)$, and $t_{3}:=\operatorname{ta}\left(c_{0}, c_{3}\right)$. If the relation $R_{c_{3}, k_{3}} R_{c_{2}, k_{2}} R_{c_{1}, k_{1}} R_{c_{0}, k_{0}}=1$ 
holds in $\mathrm{SU} V$, then

$$
\operatorname{det}\left[\begin{array}{ccc}
1 & t_{3}\left(k_{3}-\bar{k}_{3}\right)+\bar{k}_{3} & \left(t_{2}\left(\bar{k}_{2}-k_{2}\right)+k_{2}\right) \bar{k}_{0} \\
t_{3}\left(\bar{k}_{3}-k_{3}\right)+k_{3} & 1 & t_{1}\left(k_{1}-\bar{k}_{1}\right)+\bar{k}_{1} \\
\left(t_{2}\left(k_{2}-\bar{k}_{2}\right)+\bar{k}_{2}\right) k_{0} & t_{1}\left(\bar{k}_{1}-k_{1}\right)+k_{1} & 1
\end{array}\right]=0
$$

Conversely, given $0 \leqslant t_{1}, t_{2}, t_{3} \leqslant 1$ (the case $\sigma=1$ ) or $1 \leqslant t_{1}, t_{2}, t_{3}$ (the case $\sigma=-1$ ) satisfying (3.2), there exist $c_{j}$ 's subject to the relations $R_{c_{3}, k_{3}} R_{c_{2}, k_{2}} R_{c_{1}, k_{1}} R_{c_{0}, k_{0}}=1$ in $\mathrm{SU} V, t_{1}=\operatorname{ta}\left(c_{0}, c_{1}\right), t_{2}=$ $\operatorname{ta}\left(R_{c_{1}, k_{1}} c_{0}, c_{2}\right)$, and $t_{3}=\operatorname{ta}\left(c_{0}, c_{3}\right)$. Such $c_{0}, c_{1}, c_{2}, c_{3} \in \mathrm{B} V$ are unique up to the action of $\mathrm{U} V$ if $k_{j} \neq \pm 1$ and $t_{j} \neq 0$ for all $j$.

Proof. We assume that $\left\langle c_{j}, c_{j}\right\rangle=1$ for all $j$. Denote $a_{j}:=\sqrt{t}_{j}$ and choose representatives of $c_{1}, c_{3}$ such that $\left\langle c_{0}, c_{j}\right\rangle=a_{j}$ for all $j=1,3$. Let $p_{2}:=R_{c_{3}, k_{3}}^{-1} c_{0}$ and $p_{1}:=R_{c_{1}, k_{1}} c_{0}$. We choose a representative of $c_{2}$ such that $\left\langle p_{1}, c_{2}\right\rangle=a_{2}$. By Remark 2.2, the Gram matrix of the chosen $p_{2}, c_{3}, c_{0}, c_{1}, p_{1}, c_{2} \in V$ equals

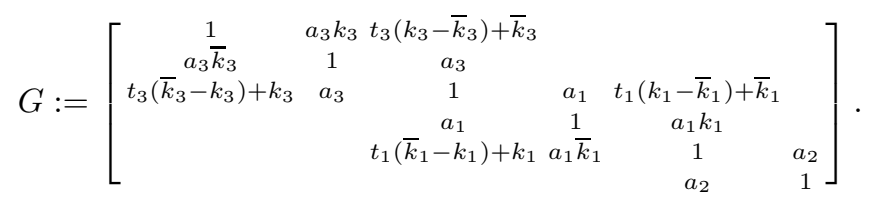

Since $R_{c_{3}, k_{3}} R_{c_{2}, k_{2}} R_{c_{1}, k_{1}} R_{c_{0}, k_{0}} c_{0}=c_{0}$ is equivalent to $R_{c_{2}, k_{2}} p_{1}=k_{0} p_{2}$ in these terms, we conclude by Remark 2.2 that

$$
G=\left[\begin{array}{cccccc}
1 & a_{3} k_{3} & t_{3}\left(k_{3}-\bar{k}_{3}\right)+\bar{k}_{3} & * & \left(t_{2}\left(\bar{k}_{2}-k_{2}\right)+k_{2}\right) \bar{k}_{0} & a_{2} \bar{k}_{0} \bar{k}_{2} \\
a_{3} \bar{k}_{3} & 1 & a_{3} & * & * & * \\
t_{3}\left(\bar{k}_{3}-k_{3}\right)+k_{3} & a_{3} & 1 & a_{1} & t_{1}\left(k_{1}-\bar{k}_{1}\right)+\bar{k}_{1} & * \\
* & * & a_{1} & 1 & a_{1} k_{1} & * \\
\left(t_{2}\left(k_{2}-\bar{k}_{2}\right)+\bar{k}_{2}\right) k_{0} & * & t_{1}\left(\bar{k}_{1}-k_{1}\right)+k_{1} & a_{1} \bar{k}_{1} & 1 & a_{2} \\
a_{2} k_{0} k_{2} & * & * & * & a_{2} & 1
\end{array}\right] .
$$

The matrix $M:=\left[\begin{array}{ccc}1 & t_{3}\left(k_{3}-\bar{k}_{3}\right)+\bar{k}_{3} & \left(t_{2}\left(\bar{k}_{2}-k_{2}\right)+k_{2}\right) \bar{k}_{0} \\ t_{3}\left(\bar{k}_{3}-k_{3}\right)+k_{3} & 1 & t_{1}\left(k_{1}-\bar{k}_{1}\right)+\bar{k}_{1} \\ \left(t_{2}\left(k_{2}-\bar{k}_{2}\right)+\bar{k}_{2}\right) k_{0} & t_{1}\left(\bar{k}_{1}-k_{1}\right)+k_{1} & 1\end{array}\right]$ is a submatrix of $G$. It is the Gram matrix of $p_{2}, c_{0}, p_{1} \in V$; since $\operatorname{dim}_{\mathbb{C}} V=2$, these points must be linearly dependent and their Gram matrix, degenerate. Thus, we arrive at (3.2).

In order to prove the uniqueness, we can assume without loss of generality that the $c_{j}$ 's are normalized so that (3.3) is the Gram matrix of $p_{2}, c_{3}, c_{0}, c_{1}, p_{1}, c_{2}$. Note that the points $p_{2}, c_{0}, p_{1} \in V$ whose Gram matrix equals $M$ are unique up to the action of $\mathrm{U} V$. Indeed, the claim is easy if $t_{j} \neq 1$ for some $1 \leqslant j \leqslant 3$ because, in this case, $\left|u_{j}\right| \neq 1$, where $u_{j}:=t_{j}\left(\bar{k}_{j}-k_{j}\right)+k_{j}$. If $t_{1}=t_{2}=t_{3}=1$, then the points $p_{2}, c_{0}, p_{1}$ coincide in $\mathrm{B} V$ and, again, the claim follows.

Consider the Gram matrices

$$
\begin{gathered}
M_{3}:=\left[\begin{array}{ccc}
1 & a_{3} k_{3} & t_{3}\left(k_{3}-\bar{k}_{3}\right)+\bar{k}_{3} \\
a_{3} \bar{k}_{3} & 1 & a_{3} \\
t_{3}\left(\bar{k}_{3}-k_{3}\right)+k_{3} & a_{3} & 1
\end{array}\right], \quad M_{1}:=\left[\begin{array}{ccc}
1 & a_{1} & t_{1}\left(k_{1}-\bar{k}_{1}\right)+\bar{k}_{1} \\
a_{1} & 1 & a_{1} k_{1} \\
t_{1}\left(\bar{k}_{1}-k_{1}\right)+k_{1} & a_{1} \bar{k}_{1} & 1
\end{array}\right], \\
M_{2}:=\left[\begin{array}{ccc}
1 & \left(t_{2}\left(\bar{k}_{2}-k_{2}\right)+k_{2}\right) \bar{k}_{0} & a_{2} \bar{k}_{0} \bar{k}_{2} \\
\left(t_{2}\left(k_{2}-\bar{k}_{2}\right)+\bar{k}_{2}\right) k_{0} & 1 & a_{2} \\
a_{2} k_{0} k_{2} & a_{2} & 1
\end{array}\right]
\end{gathered}
$$

of the triples $p_{2}, c_{3}, c_{0}, c_{0}, c_{1}, p_{1}$, and $p_{2}, p_{1}, c_{2}$. Given $p_{2}, c_{0}, p_{1} \in V$ with the Gram matrix $M$, the point $c_{j} \in V$ is uniquely determined by $M_{j}$ if $\left|u_{j}\right| \neq 1$. Yet, $c_{j}$ is uniquely determined by $M_{j}$ if $a_{j}=1$. It remains to observe that $\left|u_{j}\right|=1$ is equivalent to $t_{j}=0,1$ or $k_{j}= \pm 1$. 
Finally, let us show the existence of $c_{j}$ 's. Suppose that (3.2) is valid, $\operatorname{det} M=0$. Since $\left|u_{j}\right|^{2}-1=$ $2 t_{j}\left(t_{j}-1\right)\left(1-\operatorname{Re} k_{j}^{2}\right)$, we have $\left|u_{j}\right| \geqslant 1$ if $\sigma=-1$ and $\left|u_{j}\right| \leqslant 1$ if $\sigma=1$. This means that there exist points $p_{2}, c_{0}, p_{1} \in V$ whose Gram matrix equals $M$. These points generate $V$ if $\operatorname{rk} M=2$ and coincide in $\mathrm{B} V$ if $\operatorname{rk} M=1$. For a similar reason, the equalities $\operatorname{det} M_{3}=\operatorname{det} M_{1}=\operatorname{det} M_{2}=0$ imply that there exist $c_{3}, c_{1}, c_{2}$ such that $M_{3}, M_{1}, M_{2}$ are the Gram matrices of the triples $p_{2}, c_{3}, c_{0}, c_{0}, c_{1}, p_{1}$, and $p_{2}, p_{1}, c_{2}$, respectively. From $\left[k_{3} a_{3}\left(1-k_{3}^{2}\right)-1\right] M_{3}=0$, we obtain $k_{3} p_{2}+a_{3}\left(1-k_{3}^{2}\right) c_{3}-c_{0}=0$, i.e., $R_{c_{3}, k_{3}} p_{2}=c_{0}$. From $\left[k_{1} a_{1}\left(\bar{k}_{1}-k_{1}\right)-1\right] M_{1}=0$, we deduce $k_{1} c_{0}+a_{1}\left(\bar{k}_{1}-k_{1}\right) c_{1}-p_{1}=0$, i.e., $R_{c_{1}, k_{1}} c_{0}=p_{1}$. It follows from $\left[k_{0}-k_{2} a_{2}\left(k_{2}-\bar{k}_{2}\right)\right] M_{2}=0$ that $k_{0} p_{2}-k_{2} p_{1}+a_{2}\left(k_{2}-\bar{k}_{2}\right) c_{2}=0$, i.e., $R_{c_{2}, k_{2}} p_{1}=k_{0} p_{2}$. As $R_{c_{0}, k_{0}} c_{0}=\bar{k}_{0} c_{0}$, we obtain $R_{c_{3}, k_{3}} R_{c_{2}, k_{2}} R_{c_{1}, k_{1}} R_{c_{0}, k_{0}} c_{0}=c_{0}$. Taking into account $R_{c_{3}, k_{3}} R_{c_{2}, k_{2}} R_{c_{1}, k_{1}} R_{c_{0}, k_{0}} \in \mathrm{SU} V$ and $\left\langle c_{0}, c_{0}\right\rangle \neq 0$, we arrive at $R_{c_{3}, k_{3}} R_{c_{2}, k_{2}} R_{c_{1}, k_{1}} R_{c_{0}, k_{0}}=1$

The relation $R_{c_{3}, k_{3}} R_{c_{2}, k_{2}} R_{c_{1}, k_{1}} R_{c_{0}, k_{0}}=1$ in $\mathrm{PU} V$ means $R_{c_{3}, k_{3}} R_{c_{2}, k_{2}} R_{c_{1}, k_{1}} R_{c_{0}, k_{0}}= \pm 1$ at the level of SU $V$. Given some unitary complex numbers $k_{0}, k_{1}, k_{2}, k_{3} \in \mathbb{C}$, the next two lemmas concern the solutions, at the level of SU $V$, of the equations $R_{c_{3}, k_{3}} R_{c_{2}, k_{2}} R_{c_{1}, k_{1}} R_{c_{0}, k_{0}}= \pm 1$. (Note that, by Lemma 3.1 and Remark 2.2, equation (3.5) says that $R_{c_{3}, k_{3}} R_{c_{2}, k_{2}} R_{c_{1}, k_{1}} R_{c_{0}, k_{0}}=1$ for some $c_{0}, c_{1}, c_{2}, c_{3} \in \mathrm{B} V$ while equation (3.7) says that $R_{c_{3}, k_{3}} R_{c_{2}, k_{2}} R_{c_{1}, k_{1}} R_{c_{0}, k_{0}}=-1$ for some $c_{0}, c_{1}, c_{2}, c_{3} \in \mathrm{B} V$.)

3.4. Lemma. Given $\left.a_{0}, a_{1}, a_{2}, a_{3} \in\right] 0, \pi\left[\right.$, we put $k_{j}:=e^{a_{j} i}$ and $u_{j}:=t_{j}\left(\bar{k}_{j}-k_{j}\right)+k_{j}$ for all $0 \leqslant j \leqslant 3$. Then the equation

$$
1+2 \operatorname{Re}\left(\bar{k}_{0} u_{1} u_{2} u_{3}\right)=\left|u_{1}\right|^{2}+\left|u_{2}\right|^{2}+\left|u_{3}\right|^{2}
$$

in $t_{1}, t_{2}, t_{3} \geqslant 1$ has no solution with $t_{3}=1$ if $\sum_{j} a_{j} \leqslant \pi$ or $3 \pi \leqslant \sum_{j} a_{j}$.

Proof. Since $u_{3}=\bar{k}_{3}$ when $t_{3}=1$, the equation takes the form $2 \operatorname{Re}\left(\bar{k}_{0} \bar{k}_{3} u_{1} u_{2}\right)=\left|u_{1}\right|^{2}+\left|u_{2}\right|^{2}$, which is equivalent to $\left|u_{2}-\bar{u}_{1} k_{0} k_{3}\right|^{2}=0$, i.e., to $u_{2}=\bar{u}_{1} k_{0} k_{3}$. Note that, replacing $a_{j}$ by $\pi-a_{j}$, we change $k_{j}$ by $-\bar{k}_{j}$ and the last equation becomes $-\bar{u}_{2}=-u_{1} \bar{k}_{0} \bar{k}_{3}$, i.e., it remains the same. Therefore, we may assume that $\sum_{j} a_{j} \leqslant \pi$.

Denote $s:=\sin \left(a_{0}+a_{3}\right), b:=\cos \left(a_{0}+a_{3}\right), s_{j}:=\sin a_{j}$, and $b_{j}:=\cos a_{j}$ for $j=1,2$. It follows from $\sum_{j} a_{j} \leqslant \pi$ that $s, s_{1}, s_{2}>0$. In these terms, the equation $u_{2}=\bar{u}_{1} k_{0} k_{3}$ takes the form $-2 s_{2} t_{2} i+b_{2}+s_{2} i=$ $\left(2 s_{1} t_{1} i+b_{1}-s_{1} i\right)(b+s i)$. Hence, $b_{2}=b_{1} b+s_{1} s\left(1-2 t_{1}\right)$ and $s_{2}\left(1-2 t_{2}\right)=b_{1} s+s_{1} b\left(2 t_{1}-1\right)$. This means that $2 t_{1}-1=\frac{b_{1} b-b_{2}}{s_{1} s}$ and $2 t_{2}-1=\frac{b_{2} b-b_{1}}{s_{2} s}$ because $b^{2}+s^{2}=1$. Since $s_{1} s, s_{2} s>0$, from $t_{1}, t_{2} \geqslant 1$, we conclude that $b_{1} b-s_{1} s \geqslant b_{2}$ and $b_{2} b-s_{2} s \geqslant b_{1}$, i.e., $\operatorname{Re}\left(k_{0} k_{1} k_{3}\right) \geqslant \operatorname{Re} k_{2}$ and $\operatorname{Re}\left(k_{0} k_{2} k_{3}\right) \geqslant \operatorname{Re} k_{1}$. In view of $\sum_{j} a_{j} \leqslant \pi$, we obtain $a_{0}+a_{1}+a_{3} \leqslant a_{2}$ and $a_{0}+a_{2}+a_{3} \leqslant a_{1}$, implying $a_{1}<a_{2}$ and $a_{2}<a_{1}$, a contradiction

3.6. Lemma. Given $\left.a_{0}, a_{1}, a_{2}, a_{3} \in\right] 0, \pi\left[\right.$, we put $k_{j}:=e^{a_{j} i}$ and $u_{j}:=t_{j}\left(\bar{k}_{j}-k_{j}\right)+k_{j}$ for all $0 \leqslant j \leqslant 3$. Then the solutions of the equation

$$
1-2 \operatorname{Re}\left(\bar{k}_{0} u_{1} u_{2} u_{3}\right)=\left|u_{1}\right|^{2}+\left|u_{2}\right|^{2}+\left|u_{3}\right|^{2}
$$

in $t_{1}, t_{2}, t_{3} \geqslant 1$ constitute a compact.

Proof. We take $\sigma=-1$. Let $t$ be the tance that corresponds to the distance $d$. It follows from $\cosh ^{2} \frac{\operatorname{dist}\left(p_{1}, p_{2}\right)}{2}=\operatorname{ta}\left(p_{1}, p_{2}\right)$ that $(2 t-1)^{2}$ is the tance that corresponds to the distance $2 d$. Consequently, $64 t\left(t-\frac{1}{2}\right)^{2}(t-1)+1=\left(2(2 t-1)^{2}-1\right)^{2}$ is the tance that corresponds to the distance $4 d$.

By Lemma 3.1 and Remark 2.2, the equation (3.7) says that, for some points $c_{0}, c_{1}, c_{2}, c_{3} \in \mathrm{B} V$, the relation $R_{c_{3}, k_{3}} R_{c_{2}, k_{2}} R_{c_{1}, k_{1}} R_{c_{0},-k_{0}}=1$ holds in SUV, where $t_{1}:=\operatorname{ta}\left(c_{0}, c_{1}\right)=\operatorname{ta}\left(c_{1}, p_{1}\right), t_{2}:=$ $\operatorname{ta}\left(p_{1}, c_{2}\right)=\operatorname{ta}\left(c_{2}, p_{2}\right), t_{3}:=\operatorname{ta}\left(p_{2}, c_{3}\right)=\operatorname{ta}\left(c_{3}, c_{0}\right), p_{1}:=R_{c_{1}, k_{1}} c_{0}$, and $p_{2}:=R_{c_{3}, k_{3}}^{-1} c_{0}$. Denoting the corresponding distances by $d_{1}, d_{2}, d_{3}$, we obtain $2 d_{1}+2 d_{2} \geqslant \operatorname{dist}\left(p_{2}, c_{0}\right)$. So, if $d_{2}=\max \left(d_{1}, d_{2}\right)$, 
then $4 d_{2} \geqslant \operatorname{dist}\left(p_{2}, c_{0}\right)$. By Remark 2.2, $\operatorname{ta}\left(p_{2}, c_{0}\right)=\left|t_{3}\left(\bar{k}_{3}-k_{3}\right)+k_{3}\right|^{2}=2 t_{3}\left(t_{3}-1\right)\left(1-\operatorname{Re} k_{3}^{2}\right)+1$. Therefore, $32\left(t_{2}-\frac{1}{2}\right)^{2} t_{2}\left(t_{2}-1\right) \geqslant t_{3}\left(t_{3}-1\right)\left(1-\operatorname{Re} k_{3}^{2}\right)$. Since $\left(t_{2}-\frac{1}{2}\right)^{2} \geqslant t_{2}\left(t_{2}-1\right)$ and $t_{3}\left(t_{3}-1\right) \geqslant$ $\left(t_{3}-1\right)^{2}$, we obtain $t_{2}-\frac{1}{2} \geqslant\left(t_{3}-1\right)^{\frac{1}{2}}\left(\frac{1-\operatorname{Re} k_{3}^{2}}{32}\right)^{\frac{1}{4}}$, implying $\max \left(t_{1}, t_{2}\right) \geqslant m\left(t_{3}-1\right)^{\frac{1}{2}}$, where $m:=$ $\min \left(\left(\frac{1-\operatorname{Re} k_{1}^{2}}{32}\right)^{\frac{1}{4}},\left(\frac{1-\operatorname{Re} k_{2}^{2}}{32}\right)^{\frac{1}{4}},\left(\frac{1-\operatorname{Re} k_{3}^{2}}{32}\right)^{\frac{1}{4}}\right)>0$.

Without loss of generality, we may assume that $t_{3} \geqslant t_{2} \geqslant t_{1} \geqslant 1$.

Suppose that the set of solutions of (3.7) is not compact. Then there exist solutions with arbitrary big $t_{3}$, i.e., $t_{3} \gg 0$. The inequality $\max \left(t_{1}, t_{2}\right) \geqslant m\left(t_{3}-1\right)^{\frac{1}{2}}$ implies $t_{2} \gg 0$.

Denote $s_{j}:=\sin a_{j}, b_{j}:=\cos a_{j}$, and $x_{j}:=s_{j}\left(2 t_{j}-1\right)$. Then $\bar{k}_{0}=b_{0}-s_{0} i, u_{j}=b_{j}-x_{j} i$, and $x_{j} \geqslant s_{j}>0$ because $t_{j} \geqslant 1$ and $\left.a_{j} \in\right] 0, \pi\left[\right.$. Note that $t_{2} \gg 0$ and $t_{3} \gg 0$ imply $x_{2} \gg 0$ and $x_{3} \gg 0$. In the introduced terms, the equation (3.7) takes the form

$$
\begin{gathered}
1-2 b_{0} b_{1} b_{2} b_{3}+2 s_{0}\left(b_{2} b_{3} x_{1}+b_{3} b_{1} x_{2}+b_{1} b_{2} x_{3}\right)+2 b_{0}\left(b_{3} x_{1} x_{2}+b_{1} x_{2} x_{3}+b_{2} x_{3} x_{1}\right)-2 s_{0} x_{1} x_{2} x_{3}= \\
=b_{1}^{2}+x_{1}^{2}+b_{2}^{2}+x_{2}^{2}+b_{3}^{2}+x_{3}^{2},
\end{gathered}
$$

which can be written as

$$
x_{1}^{2}+2 p\left(x_{2}, x_{3}\right) x_{1}+q\left(x_{2}, x_{3}\right)=0,
$$

where

$$
\begin{gathered}
p\left(x_{2}, x_{3}\right):=s_{0} x_{2} x_{3}-b_{0} b_{3} x_{2}-b_{0} b_{2} x_{3}-s_{0} b_{2} b_{3}=s_{0}\left(x_{2}-b_{0} b_{2} s_{0}^{-1}\right)\left(x_{3}-b_{0} b_{3} s_{0}^{-1}\right)-b_{2} b_{3} s_{0}^{-1}, \\
q\left(x_{2}, x_{3}\right):=x_{2}^{2}-2 b_{0} b_{1} x_{2} x_{3}+x_{3}^{2}-2 s_{0} b_{1} b_{3} x_{2}-2 s_{0} b_{1} b_{2} x_{3}+2 b_{0} b_{1} b_{2} b_{3}+b_{1}^{2}+b_{2}^{2}+b_{3}^{2}-1 .
\end{gathered}
$$

It follows from $x_{2} \gg 0$ and $x_{3} \gg 0$ that $p\left(x_{2}, x_{3}\right) \gg 0$. Hence, the fact that the equation (3.8) possesses a root $x_{1} \geqslant s_{1}$ implies $s_{1}^{2}+2 p\left(x_{2}, x_{3}\right) s_{1}+q\left(x_{2}, x_{3}\right) \leqslant 0$. Consequently, we obtain

$$
\begin{gathered}
s_{1}^{2}+2 s_{0} s_{1} x_{2} x_{3}-2 b_{0} s_{1} b_{3} x_{2}-2 b_{0} s_{1} b_{2} x_{3}-2 s_{0} s_{1} b_{2} b_{3}+ \\
+x_{2}^{2}-2 b_{0} b_{1} x_{2} x_{3}+x_{3}^{2}-2 s_{0} b_{1} b_{3} x_{2}-2 s_{0} b_{1} b_{2} x_{3}+2 b_{0} b_{1} b_{2} b_{3}+b_{1}^{2}+b_{2}^{2}+b_{3}^{2}-1 \leqslant 0,
\end{gathered}
$$

which is equivalent to

$2\left(1+s_{0} s_{1}-b_{0} b_{1}\right) x_{2} x_{3}+\left(x_{2}-x_{3}\right)^{2}-2\left(b_{0} s_{1}+s_{0} b_{1}\right)\left(b_{3} x_{2}+b_{2} x_{3}\right)+2\left(b_{0} b_{1}-s_{0} s_{1}\right) b_{2} b_{3}+b_{1}^{2}+b_{2}^{2}+b_{3}^{2}+s_{1}^{2}-1 \leqslant 0$, i.e., to

$$
2\left(1-\operatorname{Re}\left(k_{0} k_{1}\right)\right) x_{2} x_{3}+\left(x_{2}-x_{3}\right)^{2}-2 \operatorname{Im}\left(k_{0} k_{1}\right)\left(b_{3} x_{2}+b_{2} x_{3}\right)+2 \operatorname{Re}\left(k_{0} k_{1}\right) b_{2} b_{3}+b_{2}^{2}+b_{3}^{2} \leqslant 0 .
$$

Since $1-\operatorname{Re}\left(k_{0} k_{1}\right)>0$ and $x_{2}, x_{3} \gg 0$, we arrive at a contradiction

\section{The space $S\left(a_{0}, a_{1}, a_{2}, a_{3}\right)$ of hexagons, $\sigma=-1$}

In this section, we study in detail the space $R\left(a_{0}, a_{1}, \ldots, a_{n}\right)$ of relations between elliptic isometries of fixed conjugacy classes for $n=3$.

From now on, we assume $\sigma=-1$ and the isometries are considered as elements in $\mathrm{PU} V$, unless the contrary is stated.

4.1. Definition. Let $\left.a_{0}, a_{1}, \ldots, a_{n} \in\right] 0, \pi\left[\right.$ be given. Denote by $R:=R\left(a_{0}, a_{1}, \ldots, a_{n}\right)$ the space of relations $R_{n} \ldots R_{1} R_{0}=1$ in $\mathrm{PU} V$ considered up to conjugation in $\mathrm{PU} V$, where the conjugacy classes 
of $R_{0}, R_{1}, \ldots, R_{n} \in \mathrm{PU} V$ are exactly those of $R_{c, k_{0}}, R_{c, k_{1}}, \ldots, R_{c, k_{n}}$, listed perhaps in different order, and $k_{j}:=e^{a_{j} i}$. Even if some $a_{j}$ 's coincide, we still consider the corresponding classes as different; in other words, the classes are labeled with the $j$ 's. Without loss of generality, we assume that the conjugacy class of $R_{0}$ is that of $R_{c, k_{0}}$.

One can view $R$ as a relative character variety, i.e., formed by all PU $V$-representations $\varrho: F_{n} \rightarrow \mathrm{PU} V$ of the free group $F_{n}:=\left\langle r_{0}, r_{1}, \ldots, r_{n} \mid r_{n} \ldots r_{1} r_{0}=1\right\rangle$ of rank $n$ such that the conjugacy class of $\varrho r_{0}$ is that of $R_{c, k_{0}}$ and the conjugacy class of $\varrho r_{j}$ is that of $R_{c, k_{\beta j}}$ for all $1 \leqslant j \leqslant n$, where $\beta$ is a permutation on $\{1,2, \ldots, n\}$; the representations are considered up to conjugation in PU $V$.

Also, we can interpret $R$ as the space of labeled $2 n$-gons $P$ as follows. Let $\varrho: F_{n} \rightarrow$ PU $V$ be a representation as above and let $c_{j}$ stand for the fixed point of $R_{j}:=\varrho r_{j}$. The consecutive vertices of the closed piecewise geodesic path $P$ are $c_{1}, p_{1}, c_{2}, p_{2}, \ldots, c_{n}, p_{n}:=c_{0}$, where $p_{j}:=R_{j} p_{j-1}$ (the indices are modulo $n$ ). Each vertex $c_{j}$ is labeled with some conjugacy class (of $R_{c, k_{l}}, l \neq 0$ ) and $p_{n}$ is labeled with the conjugacy class of $R_{c, k_{0}}$.

4.2. Definition. Denote by $m_{0}: F_{n} \rightarrow F_{n}$ the automorphism of $F_{n}$ given by $r_{0} \mapsto r_{1} r_{0} r_{1}^{-1}, r_{j} \mapsto r_{j+1}$ for any $0<j<n$, and $r_{n} \mapsto r_{1}$; for any $0<l<n$, denote by $m_{l}: F_{n} \rightarrow F_{n}$ the automorphism of $F_{n}$ given by $r_{l} \mapsto r_{l+1}, r_{l+1} \mapsto r_{l+1} r_{l} r_{l+1}^{-1}$, and $r_{j} \mapsto r_{j}$ for any $j \neq l, l+1$; and denote by $m_{n}: F_{n} \rightarrow F_{n}$ the automorphism of $F_{n}$ given by $r_{0} \mapsto r_{1} r_{n}^{-1} r_{0} r_{n} r_{1}^{-1}, r_{1} \mapsto r_{1} r_{n} r_{1}^{-1}, r_{j} \mapsto r_{j}$ for all $1<j \leqslant n$, and $r_{n} \mapsto r_{1}$. The group $\bar{M}_{n}$ generated by $m_{0}, m_{1}, \ldots, m_{n}$ acts from the right on $R\left(a_{0}, a_{1}, \ldots, a_{n}\right)$ by composition at the level of representations.

In terms of labeled $2 n$-gons, the action of $m_{0}$ is just replacing the label $c_{0}$ from $p_{n}$ to $p_{1}$.

Note that $m_{0} m_{l}=m_{l+1} m_{0}$ for all $0<l<n$. Also, by induction on $l$, the automorphism $m_{0}^{l}$ shifts by $l$ (modulo $n$ ) the indices of the $r_{j}$ 's for $1 \leqslant j \leqslant n$ and $m_{0}^{l}: r_{0} \mapsto\left(r_{l} \ldots r_{2} r_{1}\right) r_{0}\left(r_{l} \ldots r_{2} r_{1}\right)^{-1}$. In particular, $m_{0}^{n}=1$.

Our main interest in this section is to understand when the action of $\bar{M}_{n}$ on a component of $R$ is discrete.

4.3. Definition. Denote by $S:=S\left(a_{0}, a_{1}, \ldots, a_{n}\right)$ the quotient of $R\left(a_{0}, a_{1}, \ldots, a_{n}\right)$ by the action of the cyclic group of order $n$ generated by $m_{0}$. In other words, $S$ is formed by $2 n$-gons with a forgotten label $c_{0}$ at the $p_{j}$ 's.

Let $1 \leqslant j, l \leqslant n$ be such that $j \neq l-1, l$ (the indices are modulo $n$ ) and let $P \in S$ be $2 n$-gon with consecutive vertices $c_{1}, p_{1}, c_{2}, p_{2}, \ldots, c_{n}, p_{n}$. The modification $P m_{j, l}$ of $P$ is defined as follows. The geodesic segment $\left[p_{j}, c_{l}\right]$ cuts $P$ into two polygons $P_{1}$ and $P_{2}$ whose consecutive vertices are respectively $p_{j}, c_{j+1}, p_{j+1}, \ldots, p_{l-1}, c_{l}$ and $c_{l}, p_{l}, c_{l+1}, p_{l+1}, \ldots, c_{j}, p_{j}$. We rotate $P_{1}$ with $R_{l}$ so that $R_{l} P_{1}$ and $P_{2}$ become glued along $\left[c_{l}, p_{l}\right]$ (and remove this side), thus providing a new $2 n$-gon $P m_{j, l} \in S$ that has two new (consecutive) sides $\left[p_{k}, c_{l}\right]$ and $R_{l}\left[c_{l}, p_{j}\right]=\left[c_{l}, R_{l} p_{j}\right]$.

The group $M_{n}$ generated by all modifications $m_{j, l}$ acts from the right on $S$. It is easy to see that the map $R \rightarrow S$ is a bijection at the level of the $\bar{M}_{n}$-orbits and the $M_{n}$-orbits. (For a formal proof, one can use the relations $m_{0} m_{l}=m_{l+1} m_{0}$.)

Let $P \in S$ be a $2 n$-gon with consecutive vertices $c_{1}, p_{1}, c_{2}, p_{2}, \ldots, c_{n}, p_{n}$. Then the sides $\left[p_{j-1}, c_{j}\right]$ and $\left[c_{j}, p_{j}\right]$ have equal length and the 'exterior' angle at $c_{j}$, i.e., the angle from $\left[c_{j}, p_{j-1}\right]$ to $\left[c_{j}, p_{j}\right]$ at $c_{j}$ counted in the counterclockwise sense, equals $2 a_{j}$ (unless $p_{j-1}=c_{j}=p_{j}$ ). It follows from the relation $R_{n} \ldots R_{1} R_{0}=1$ that the 'interior' angles at the $p_{j}$ 's sum to $2 \pi-2 a_{0}$ modulo $2 \pi$. Conversely, if a $2 n$-gon $P$ satisfies the listed conditions, we obtain $P \in S$.

If $a_{j} \geqslant \frac{\pi}{2}$ for all $0 \leqslant j \leqslant n$ and the 'interior' angles at the $p_{j}$ 's sum to $2 \pi-2 a_{0}$, then $P$ is convex. In this case, the $2 n$-gon $P$ remains convex after any modification $m \in M_{n}$.

4.4. Small $n$. Clearly, $S\left(a_{0}\right)=\varnothing$ because $\left.a_{0} \in\right] 0, \pi\left[\right.$. By Remark 2.2 , the space $S\left(a_{0}, a_{1}\right)$ consists of a single point iff $a_{0}+a_{1}=\pi$; otherwise, $S\left(a_{0}, a_{1}\right)=\varnothing$. 
4.4.1. Lemma (folklore). Let $\left.a_{0}, a_{1}, a_{2} \in\right] 0, \pi\left[\right.$ be fixed. Then $S\left(a_{0}, a_{1}, a_{2}\right)$ is nonempty exactly in the following cases:

- $\sum_{j} a_{j}=\pi$ or $\sum_{j} a_{j}=2 \pi$,

- $\sum_{j} a_{j}<\pi$,

- $2 \pi<\sum_{j} a_{j}$.

In these cases, $S\left(a_{0}, a_{1}, a_{2}\right)$ consists of a single point and the corresponding quadrangle $\left(c_{1}, p_{1}, c_{2}, p_{2}\right)$ is respectively degenerate (i.e., $c_{1}=p_{1}=c_{2}=p_{2}$ ), clockwise oriented, and counterclockwise oriented.

Proof. If $c_{0}=c_{1}=c_{2}$, then $k_{0}^{2} k_{1}^{2} k_{2}^{2}=1$ by Remark 2.2 and $\sum_{j} a_{j} \equiv 0 \bmod \pi$. A bit later, we will see that the converse is also true.

It cannot happen that only two of $c_{0}, c_{1}, c_{2}$ coincide. So, we assume these points pairwise distinct. Denote by $I_{j}$ the reflection in the geodesic $\gamma_{j}$ joining $c_{j-1}$ and $c_{j}$ (the indices are modulo 3 ). Then $R_{j}=I_{j+1} I_{j}$. Indeed, it suffices to show this for $j=1$. In the quadrangle $P=\left(c_{1}, p_{1}, c_{2}, p_{2}\right)$, the triangles $\left(c_{1}, p_{2}, c_{2}\right)$ and $\left(c_{1}, p_{1}, c_{2}\right)$ are congruent by means of $I_{2}$ because $p_{1}=R_{1} p_{2} \neq p_{2}$ in view of $\left.a_{1} \in\right] 0, \pi[$ (otherwise, $R_{1}$ would have two distinct fixed points $c_{1}$ and $p_{2}=c_{0}$ ). Therefore, $I_{2} c_{0}=R_{1} c_{0}$, implying $I_{2} I_{1} c_{0}=R_{1} c_{0}$ and $I_{2} I_{1} c_{1}=R_{1} c_{1}$. The orientation-preserving isometries $I_{2} I_{1}$ and $R_{1}$ coincide on two distinct points. Hence, they are equal.

By Remark 2.3, $a_{j}=\alpha\left(\gamma_{j}, \gamma_{j+1}\right)$ is the oriented angle from $\gamma_{j}$ to $\gamma_{j+1}$. It is easy to see that, if the triangle $\left(c_{0}, c_{1}, c_{2}\right)$ is clockwise (counterclockwise) oriented, then $a_{j}$ (respectively, $\pi-a_{j}$ ) is its interior angle at $c_{j}$. So, we arrive at $\sum_{j} a_{j}<\pi$ and $2 \pi<\sum_{j} a_{j}$, respectively. Obviously, under this condition, the quadrangle $\left(c_{1}, p_{1}, c_{2}, p_{2}\right)$ is geometrically unique

4.5. Topology of $S\left(a_{0}, a_{1}, a_{2}, a_{3}\right)$. Now, we change the convention concerning labeling the vertices $c_{j}$ 's of a point $P \in S\left(a_{0}, a_{1}, a_{2}, a_{3}\right)$ so that $c_{j}$ and $R_{j}$ correspond to the conjugacy class of $R_{c, k_{j}}$ for all $1 \leqslant$ $j \leqslant 3$. Thus, we have two types of hexagons: those with consecutive vertices $c_{1}, p_{1}, c_{2}, p_{2}, c_{2}, p_{3}$, where $p_{1}=R_{1} p_{3}, p_{2}=R_{2} p_{1}$, and $p_{3}=R_{3} p_{2}$, and those with consecutive vertices $p_{3}, c_{3}, p_{2}, c_{2}, p_{1}, c_{1}$, where $p_{1}=R_{1}^{-1} p_{3}, p_{2}=R_{2}^{-1} p_{1}$, and $p_{3}=R_{3}^{-1} p_{2}$. To each hexagon $P$, we associate a triple $\left(t_{1}, t_{2}, t_{3}\right) \in \mathbb{R}^{3}$, where $t_{j}:=\operatorname{ta}\left(c_{j}, p_{j}\right)$. In what follows, we frequently use to place the label $c_{0}$ at $p_{3}$ thus getting the relation $R_{3} R_{2} R_{1} R_{0}=1$ for the hexagons of the first type and the relation $R_{0} R_{1} R_{2} R_{3}=1$ for the hexagons of the second type.

According to the new convention, the group $M_{3}$, previously generated by $m_{1,3}, m_{2,1}, m_{3,2}$ is now generated by the modifications $n_{1}, n_{2}, n_{3}$ that transform respectively the relation $R_{3} R_{2} R_{1} R_{0}=1$ into the relations $R_{0}^{R_{3}^{-1}} R_{1} R_{2}^{R_{1}^{-1}} R_{3}=1, R_{0}^{R_{3}^{-1}} R_{1}^{R_{2}} R_{2} R_{3}=1, R_{0}^{R_{1}} R_{1} R_{2}^{R_{3}} R_{3}=1$ and the relation $R_{0} R_{1} R_{2} R_{3}=1$ into the relations $R_{3} R_{2}^{R_{1}} R_{1} R_{0}^{R_{3}}=1, R_{3} R_{2} R_{1}^{R_{2}^{-1}} R_{0}^{R_{3}}=1, R_{3} R_{2}^{R_{3}^{-1}} R_{1} R_{0}^{R_{1}^{-1}}=1$, thus altering the type of a hexagon. It is easy to verify that $n_{j}^{2}=1$ for all $j=1,2,3$ and that $n_{j}$ keeps all the $t_{l}$ 's except perhaps the $t_{j}$.

We say that a point $P \in S\left(a_{0}, a_{1}, a_{2}, a_{3}\right)$ is degenerate with respect to $t_{3}$ if the only point $P^{\prime} \in$ $S\left(a_{0}, a_{1}, a_{2}, a_{3}\right)$ of the same type as $P$ such that $t_{3} P^{\prime}=t_{3} P$ is the point $P$. This implies that $t_{3} P=1$ or $c_{1}=c_{2}$ for $P$ as, otherwise, we get infinitely many points with the same $t_{3}$ provided by $p_{3}, g c_{1}, g c_{2}, c_{3}$, where $g \in C_{R}$ runs over the centralizer $C_{R}$ in PUV of $R:=R_{0} R_{3}$ (when $P$ is of the first type) or of $R:=R_{3} R_{0}$ (when $P$ is of the second type) and the label $c_{0}$ is placed at $p_{3}$. A point $P \in S\left(a_{0}, a_{1}, a_{2}, a_{3}\right)$ is said to be boundary with respect to $t_{3}$ if $P$ is not degenerate with respect to $t_{3}$ and $t_{3} P=1$. A point $P \in S\left(a_{0}, a_{1}, a_{2}, a_{3}\right)$ is regular with respect to $t_{3}$ if it is not degenerate nor boundary with respect to $t_{3}$.

4.5.1. Lemma. A point $P \in S\left(a_{0}, a_{1}, a_{2}, a_{3}\right)$ is regular with respect to $t_{3}$ iff $t_{3} P \neq 1$ and $c_{1} \neq c_{2}$ for $P$.

It follows a complete list of the points in $S\left(a_{0}, a_{1}, a_{2}, a_{3}\right)$ degenerate or boundary with respect to $t_{3}$ : a. Let $\sum_{j} a_{j}<\pi$ or $3 \pi<\sum_{j} a_{j}$. Then there exist exactly 2 degenerate points $P_{1}$ and $P_{2}$ of a given type. These points satisfy $t_{3} P_{1}=1, c_{1} \neq c_{2}$ for $P_{1}, t_{3} P_{2} \neq 1$, and $c_{1}=c_{2}$ for $P_{2}$. 
b. Let $\sum_{j} a_{j}=\pi$ or $\sum_{j} a_{j}=3 \pi$. Then there exists exactly 1 degenerate point of a given type. This point satisfies $c_{1}=p_{1}=c_{2}=p_{2}=c_{3}=p_{3}$.

c. Let $a_{0}+a_{3}<\pi<a_{1}+a_{2}$ or $a_{1}+a_{2}<\pi<a_{0}+a_{3}$. Then there exists exactly 1 degenerate point $P_{0}$ of a given type. The condition $c_{1}=p_{1}=c_{2}=p_{2}=c_{3}=p_{3}$ for $P_{0}$ is equivalent to $\sum_{j} a_{j}=2 \pi$.

d. Let $a_{0}+a_{3}=a_{1}+a_{2}=\pi$. Then there is no degenerate point. This case is the only one when there are points boundary with respect to $t_{3}$. Such points, forming a space homeomorphic to the ray $[1, \infty[$, are given by the condition $t_{3} P=1$.

e. There is no degenerate point in the remaining cases, i.e., in the cases

- $\pi<\sum_{j} a_{j}$ with $a_{0}+a_{3}<\pi$ and $a_{1}+a_{2}<\pi$,

- $a_{0}+a_{3}=\pi$ with $a_{1}+a_{2} \neq \pi$,

- $\sum_{j} a_{j}<3 \pi$ with $\pi<a_{0}+a_{3}$ and $\pi<a_{1}+a_{2}$,

- $a_{1}+a_{2}=\pi$ with $a_{0}+a_{3} \neq \pi$.

Proof. If $t_{3} P=1$ and $c_{1} \neq c_{2}$ for $P$, then, by Lemma 4.4.1, $P$ is degenerate with respect to $t_{3}$. If $t_{3} P \neq 1, c_{1}=c_{2}$ for $P$, and $P^{\prime}$ is a point of the same type as $P$ with $t_{3} P^{\prime}=t_{3} P$, then we can assume that $P$ and $P^{\prime}$ share the vertices $c_{3}, p_{3}$; so, the vertices $c_{3}, p_{3}, c_{1}=c_{2}$ determine $P$ and the vertices $c_{3}, p_{3}, c_{1}^{\prime}, c_{2}^{\prime}$ determine $P^{\prime}$. Placing the label $c_{0}$ at $p_{3}$, we get the relations $\left(R_{2} R_{1}\right) R_{0} R_{3}=1$ for $P$ and $R_{2}^{\prime} R_{1}^{\prime} R_{0} R_{3}=1$ for $P^{\prime}$ in the case of the first type, and the relations $\left(R_{1} R_{2}\right) R_{3} R_{0}=1$ for $P$ and $R_{1}^{\prime} R_{2}^{\prime} R_{3} R_{0}=1$ for $P^{\prime}$ in the case of the second type. Since $c_{0} \neq c_{3}$ implies $a_{1}+a_{2} \neq \pi$, the relation $R_{2}^{\prime} R_{1}^{\prime}\left(R_{2} R_{1}\right)^{-1}=1$ or $R_{1}^{\prime} R_{2}^{\prime}\left(R_{1} R_{2}\right)^{-1}=1$, respectively, provides $c_{1}=c_{1}^{\prime}=c_{2}^{\prime}$ by Lemma 4.4.1. Thus, $P$ is degenerate with respect to $t_{3}$ if $t_{3} P=1$ and $c_{1} \neq c_{2}$ for $P$ or if $t_{3} P \neq 1$ and $c_{1}=c_{2}$ for $P$.

Since $t_{3} P \neq 1$ and $c_{1}=c_{2}$ for $P$ imply $a_{1}+a_{2} \neq \pi$, by Lemma 4.4.1, there exists a (unique) point $P \in S\left(a_{0}, a_{1}, a_{2}, a_{3}\right)$ of a given type with $t_{3} P \neq 1$ and $c_{1}=c_{2}$ (hence, degenerate with respect to $\left.t_{3}\right)$ exactly in the following cases

$\bullet \sum_{j} a_{j}<\pi, \quad \bullet \sum_{j} a_{j}<2 \pi$ and $\pi<a_{1}+a_{2}, \quad \bullet 2 \pi<\sum_{j} a_{j}$ and $a_{1}+a_{2}<\pi, \quad \bullet 3 \pi<\sum_{j} a_{j}$.

Let $a_{0}+a_{3} \neq \pi$. Again by Lemma 4.4.1, the relation $R_{2} R_{1} R_{0} R_{3}=1$ or $R_{1} R_{2} R_{3} R_{0}=1$, respectively, together with $t_{3} P=1$ determine uniquely a point $P \in S\left(a_{0}, a_{1}, a_{2}, a_{3}\right)$ of a given type. So, $P$ is degenerate with respect to $t_{3}$ when $t_{3} P=1$. Moreover, by Lemma 4.4.1, there exists a (unique) point $P_{0}$ of a given type with $t_{3} P_{0}=1$ (hence, degenerate with respect to $t_{3}$ ) exactly in the following cases

- $\sum_{j} a_{j}=\pi, \quad \bullet \sum_{j} a_{j}=2 \pi$ and $a_{0}+a_{3} \neq \pi, \quad \bullet \sum_{j} a_{j}=3 \pi$,

- $\sum_{j} a_{j}<2 \pi$ and $\pi<a_{0}+a_{3}, \quad \bullet 2 \pi<\sum_{j} a_{j}$ and $a_{0}+a_{3}<\pi$

- $\sum_{j} a_{j}<\pi$

- $3 \pi<\sum_{j} a_{j}$.

Note that $c_{1}=c_{2}$ with $t_{3} P=1$ is possible exactly in the first 3 cases because $a_{0}+a_{3} \neq \pi$. In these cases, $c_{1}=p_{1}=c_{2}=p_{2}=c_{3}=p_{3}$.

In the case $a_{0}+a_{3}=\pi \neq a_{1}+a_{2}$, there is no point $P$ with $t_{3} P=1$.

Let $a_{0}+a_{3}=a_{1}+a_{2}=\pi$. Then $t_{3} P=1$ implies $c_{0}=c_{3}$ and $c_{1}=c_{2}$. Now $t_{1} P=t_{2} P$ can be an arbitrary number in $\left[1, \infty\left[\right.\right.$. In other words, the condition $t_{3} P=1$ provides a point boundary with respect to $t_{3}$. In this way, we listed all possible points boundary with respect to $t_{3}$.

Summarizing, we arrive at the list in the lemma

Assuming the label $c_{0}$ placed at $p_{3}$, to every point $P \in S\left(a_{0}, a_{1}, a_{2}, a_{3}\right)$, we associate the isometry $R:=R_{0} R_{3}$ if $P$ is of the first type and the isometry $R:=R_{3} R_{0}$, if $P$ is of the second type. It follows immediately from Lemma 4.5.1 that the isometry $R$ is elliptic (or the identity) if $P$ is degenerate or boundary with respect to $t_{3}$.

In the following proposition, for any point $P \in S\left(a_{0}, a_{1}, a_{2}, a_{3}\right)$, we place the label $c_{0}$ at $p_{3}$.

4.5.2. Proposition. It follows a full list of connected components of $S:=S\left(a_{0}, a_{1}, a_{2}, a_{3}\right)$ of a given (either) type presented with respect to the cases listed in Lemma 4.5.1:

a. In this case, there are two components. One is topologically a 2-sphere and the isometry $R_{0} R_{3}$ is elliptic for every point in this component. The other component is topologically a plane and the isometry $R_{0} R_{3}$ is hyperbolic for every point in this component. 
b. In this case, there are two components. One is a single point, degenerate with respect to $t_{3}$, and the isometry $R_{0} R_{3}$ is elliptic for this point. The other component is topologically a plane and the isometry $R_{0} R_{3}$ is hyperbolic for every point in this component.

c. In this case, there is a unique component, topologically a plane. There are points in the component whose isometry $R_{0} R_{3}$ is elliptic, parabolic, or hyperbolic.

d. In this case, there is a unique component, topologically a plane. The isometry $R_{0} R_{3}$ is hyperbolic or the identity for every point in this component.

e. In this case, there is a unique component, topologically a plane. The isometry $R_{0} R_{3}$ is hyperbolic for every point in this component.

Every component $C$, except of the one consisting of a single point, contains a curve $L_{2} \subset C$ dividing $C$ into 2 parts such that $t_{3} C=t_{3} L_{2}$ and every fibre of $L_{2} \stackrel{t_{3}}{\longrightarrow} \mathbb{R}$ contains at most 2 points with the unique exception in the case $\mathbf{d}$ where the fibre over $t_{3}=1$ is topologically a ray. The curve $L_{2}$ is topologically a circle or a line when $C$ is topologically a 2-sphere or a plane, respectively. The curve $L_{2}$ admits a smooth parameterization by $t_{3}$ at the points where the isometry $R_{0} R_{3}$ is hyperbolic.

Proof. Let $C$ be a connected component of $S$. The hexagons in $C$ are all of a same type. We will deal with the first type indicating in parentheses what happens to the case of the second one. Denote $t_{0}:=t_{0}\left(a_{0}, a_{3}\right)$ (see Remark 2.4). By Lemma 4.5.1, $t_{3} P<t_{0}$ if $P \in S$ is degenerate with respect to $t_{3}$.

Let $P \in C$. If $P$ is degenerate with respect to $t_{3}$, then the fibre of $C \stackrel{t_{3}}{\longrightarrow} \mathbb{R}$ at $P$ consists of a single point.

If $P$ is boundary with respect to $t_{3}$, then the fibre of $C \stackrel{t_{3}}{\longrightarrow} \mathbb{R}$ at $P$ is homeomorphic to the ray $[1, \infty[$ by Lemma 4.5.1.

Suppose that $P$ is regular with respect to $t_{3}$. Denote by $\gamma_{0}$ the geodesic that joins $c_{0}$ with $c_{3}$. By Remark 2.3, there exist unique geodesics $\gamma_{1}, \gamma_{2}$ such that $R_{0}=I_{1} I_{0}$ and $R_{3}=I_{0} I_{2}$ (such that $R_{0}=I_{0} I_{1}$ and $R_{3}=I_{2} I_{0}$ for the second type) because $R_{0} \neq 1$ and $R_{3} \neq 1$, where $I_{j}$ stands for the reflection in $\gamma_{j}$. Note that, by Remark 2.3, $\alpha\left(\gamma_{0}, \gamma_{1}\right)=a_{0}$ and $\alpha\left(\gamma_{2}, \gamma_{0}\right)=a_{3}$ (for the second type, $\alpha\left(\gamma_{1}, \gamma_{0}\right)=a_{0}$ and $\left.\alpha\left(\gamma_{0}, \gamma_{2}\right)=a_{3}\right)$.

Since $c_{1}$ or $c_{2}$ is not a fixed point of $R:=R_{0} R_{3}=I_{1} I_{2} \neq 1$ (of $R:=R_{3} R_{0}=I_{2} I_{1} \neq 1$ for the second type), the points $c_{0}, g c_{1}, g c_{2}, c_{3}$, where $g$ runs over $C_{R}$, provide the hexagons forming the fibre of $C \stackrel{t_{3}}{\longrightarrow} \mathbb{R}$ and this fibre is homeomorphic to the centralizer $C_{R}$. Indeed, let $I^{\prime}$ stand for the reflection in a geodesic joining $c_{1}$ and $c_{2}$ (here admitting that the points $c_{1}$ e $c_{2}$ may coincide, which is in fact impossible). Then, by Remark 2.3, $R_{1}=I^{\prime} I_{1}^{\prime}$ and $R_{2}=I_{2}^{\prime} I^{\prime}\left(R_{1}=I_{1}^{\prime} I^{\prime}\right.$ and $R_{2}=I^{\prime} I_{2}^{\prime}$ for the second type) for suitable (unique) reflections in geodesics $I_{1}^{\prime}$ and $I_{2}^{\prime}$. The relation $R_{2} R_{1} R_{0} R_{3}=1$ (the relation $R_{1} R_{2} R_{3} R_{0}=1$ for the second type) implies $I_{2}^{\prime} I_{1}^{\prime}=I_{2} I_{1} \neq 1$ (implies $I_{1}^{\prime} I_{2}^{\prime}=I_{1} I_{2} \neq 1$ for the second type). Now the assertion follows from Remark 2.3. Indeed, acting by $C_{R}$, we obtain $I_{1}^{\prime}=I_{1}$ and $I_{2}^{\prime}=I_{2}$ due to Remark 2.3. Since $c_{1} \neq c_{2}$, from $g c_{1}=c_{1}$ and $g c_{2}=c_{2}$ for $g \in C_{R}$, we conclude $g=1$.

Denote by $L_{2} \subset C$ the subset of all points $P \in C$ degenerate or boundary with respect to $t_{3}$ and of all those points $P \in C$ regular with respect to $t_{3}$ that satisfy $I_{1}^{\prime}=I_{1}$ and $I_{2}^{\prime}=I_{2}$. By Remark 2.3, the fibre of $C \stackrel{t_{3}}{\longrightarrow} \mathbb{R}$ at $P \in C$ either is a single point $P \in L_{2}$ (when $P$ is degenerate), or is included in $L_{2}$ and homeomorphic to [1, $\infty$ [ (when $P$ is boundary), or is a circle and contains exactly two points in $L_{2}$ (when $P$ is regular and $R$ is elliptic), or is a line and contains exactly one point in $L_{2}$ (when $R$ is parabolic or hyperbolic).

Any regular point $P \in L_{2}$ can be described as 4 geodesics $\gamma, \gamma_{0}, \gamma_{1}, \gamma_{2}$ such that $c_{0}=\gamma_{1} \cap \gamma_{0}$, $c_{1}=\gamma \cap \gamma_{1}, c_{2}=\gamma_{2} \cap \gamma, c_{3}=\gamma_{0} \cap \gamma_{2}, \alpha\left(\gamma_{0}, \gamma_{1}\right)=a_{0}, \alpha\left(\gamma_{1}, \gamma\right)=a_{1}, \alpha\left(\gamma, \gamma_{2}\right)=a_{2}, \alpha\left(\gamma_{2}, \gamma_{0}\right)=a_{3}$, $R_{0}=I_{1} I_{0}, R_{1}=I I_{1}, R_{2}=I_{2} I, R_{3}=I_{0} I_{2}$ (for the second type, $\alpha\left(\gamma_{1}, \gamma_{0}\right)=a_{0}, \alpha\left(\gamma, \gamma_{1}\right)=a_{1}$, $\left.\alpha\left(\gamma_{2}, \gamma\right)=a_{2}, \alpha\left(\gamma_{0}, \gamma_{2}\right)=a_{3}, R_{0}=I_{0} I_{1}, R_{1}=I_{1} I, R_{2}=I I_{2}, R_{3}=I_{2} I_{0}\right)$, where $I, I_{0}, I_{1}, I_{2}$ stand respectively for the reflections in $\gamma, \gamma_{0}, \gamma_{1}, \gamma_{2}$. 
Suppose that there exists a point $P \in L_{2}$ with hyperbolic $R$. Then, by Remark 2.5, we can continuously deform $P$ in $L_{2}$ making $t_{3}$ arbitrarily big because the tance $t_{3}=\operatorname{ta}\left(c_{0}, c_{3}\right)$ is greater or equal than that between the geodesics $\gamma_{1}$ and $\gamma_{2}$. As well, we can continuously deform $P$ diminishing $t_{3}$ till the moment when the geodesics $\gamma_{1}$ and $\gamma_{2}$ are becoming tangent. In other words, $] t_{0}, \infty\left[\subset t_{3} C\right.$ if $R$ can be hyperbolic for a point in $L_{2}$.

Suppose that there exists a point $P \in L_{2}$ with parabolic $R$. Then, by Remark 2.9 , there is a small continuous deformation of $P$ providing points in $L_{2}$ with hyperbolic $R$ as well as with elliptic $R$. Therefore, $\left.t_{0} \in\right] b, \infty\left[\subset t_{3} C\right.$ in this case. In terms of Remark 2.9, if the triangle $\left(v_{1}, v, v_{2}\right)$ is clockwise (counterclockwise, for the second type) oriented, then $a_{0}+a_{3}<\pi$ and $\pi<a_{1}+a_{2}$. If the triangle $\left(v_{1}, v, v_{2}\right)$ is counterclockwise (clockwise, for the second type) oriented, then $\pi<a_{0}+a_{3}$ and $a_{1}+a_{2}<\pi$. Hence, a point $P \in L_{2}$ with parabolic $R$ can exist only in the case $\mathbf{c}$ of Lemma 4.5.1.

Suppose that there exists a regular point $P \in L_{2}$ with elliptic $R$. Denote $a:=\alpha\left(\gamma_{2}, \gamma_{1}\right)$ (denote $a:=\alpha\left(\gamma_{1}, \gamma_{2}\right)$ for the second type) and let $c$ stand for the intersection point of the geodesics $\gamma_{1}, \gamma_{2}$. By Remark 2.7, when the triangle $\left(c, c_{0}, c_{3}\right)$ is clockwise (counterclockwise, for the second type) oriented, we have $a+\pi<a_{0}+a_{3}$, and when it is counterclockwise (clockwise, for the second type) oriented, we have $a_{0}+a_{3}<a$. Again by Remark 2.7, we obtain $a+a_{1}+a_{2}<\pi$ or $2 \pi<a+a_{1}+a_{2}$. Once again by Remark 2.7, we can continuously vary $a$ within the interval ]0, $\pi$ [, thus getting points in $L_{2}$, if we keep the two nonstrict inequalities, i.e., the inequalities in one of the following 4 variants:

- $a+\pi \leqslant a_{0}+a_{3}$ and $a+a_{1}+a_{2} \leqslant \pi$,

- $a_{0}+a_{3} \leqslant a$ and $a+a_{1}+a_{2} \leqslant \pi$,

- $a+\pi \leqslant a_{0}+a_{3}$ and $2 \pi \leqslant a+a_{1}+a_{2}$,

- $a_{0}+a_{3} \leqslant a$ and $2 \pi \leqslant a+a_{1}+a_{2}$.

(The first two pictures below illustrate the first and the fourth variants; the other two illustrate the second and the third variants.)
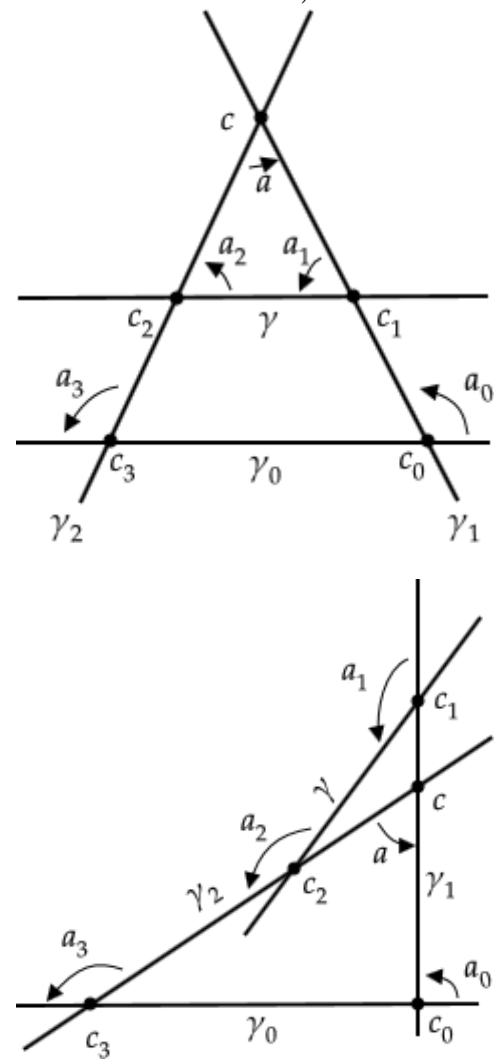
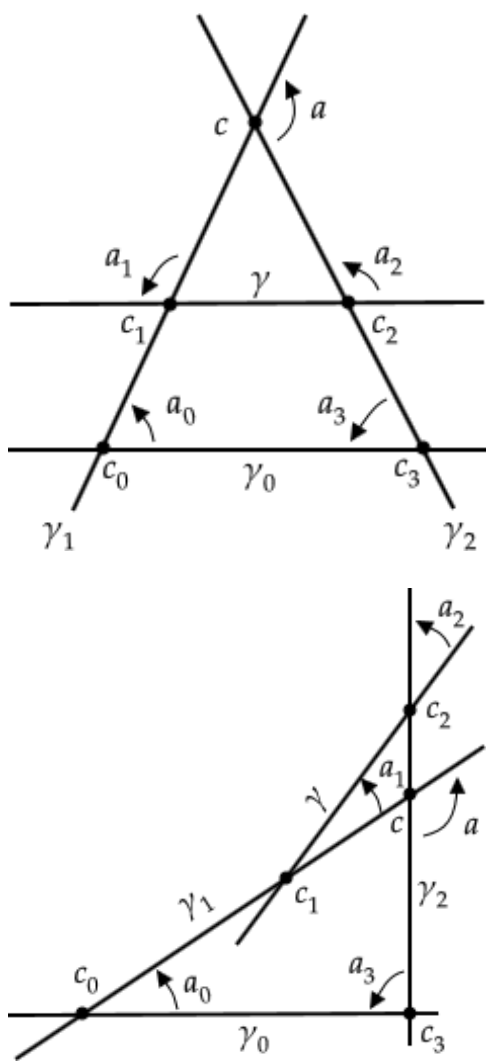
Such a deformation can be performed by varying the tance $t_{3} P$ within the interval $\left[1, t_{0}[\right.$. Note that $a$ depends monotonically on $t_{3} P$ while $t_{3} P$ varies within the interval $] 1, t_{0}\left[\right.$ and $P \in L_{2}$ remains regular of the same type. Rewriting the inequalities in a more convenient form, we arrive at

- $a \leqslant a_{0}+a_{3}-\pi$ and $a \leqslant \pi-a_{1}-a_{2}$,

- $2 \pi-a_{1}-a_{2} \leqslant a \leqslant a_{0}+a_{3}-\pi$,

- $a_{0}+a_{3} \leqslant a \leqslant \pi-a_{1}-a_{2}$,

- $a_{0}+a_{3} \leqslant a$ and $2 \pi-a_{1}-a_{2} \leqslant a$.

In either variant, there is some $0<a<\pi$ such that both inequalities are strict. This implies

- $a_{1}+a_{2}<\pi<a_{0}+a_{3}$ with $0<a_{0}+a_{3}-\pi<\pi$ and $0<\pi-a_{1}-a_{2}<\pi$,

- $3 \pi<\sum_{j} a_{j}$ with $0<2 \pi-a_{1}-a_{2}<a_{0}+a_{3}-\pi<\pi$,

- $\sum_{j} a_{j}<\pi$ with $0<a_{0}+a_{3}<\pi-a_{1}-a_{2}<\pi$,

- $a_{0}+a_{3}<\pi<a_{1}+a_{2}$ with $0<a_{1}+a_{2}-\pi<\pi$ and $0<\pi-a_{0}-a_{3}<\pi$.

In particular, for given $a_{j}$ 's, only one variant is possible, i.e., the four variants are disjoint.

In the second and third variants, we are in the case a of Lemma 4.5.1 and we can reach both values $2 \pi-a_{1}-a_{2}, a_{0}+a_{3}-\pi$ and $a_{0}+a_{3}, \pi-a_{1}-a_{2}$, respectively, by varying $a$. Since the inequalities become equalities for such points in $L_{2}$, we get by Remark 2.7 the two distinct degenerate points $P_{1}, P_{2}$ mentioned in Lemma 4.5.1 a. As $C$ is connected, we obtain $\left[t_{3} P_{1}, t_{3} P_{2}\right] \subset t_{3} C$. We claim that $\left[t_{3} P_{1}, t_{3} P_{2}\right]=t_{3} C$. Indeed, otherwise, there would exist an extra regular point $P \in L_{2}$ with elliptic $R$. The values of $a$ for such $P$ should fall into one of the four variants and, since the variants are disjoint, the variant should be the same as the one we started with. This contradicts the fact that $a$ depends monotonically on $t_{3} P$. Now we visualize $C$ as a topological 2 -sphere and $L_{2}$, as a topological circle dividing $C$ into 2 parts.

In the first and fourth variants, we act similarly. We can reach the values $\min \left(a_{0}+a_{3}-\pi, \pi-a_{1}-a_{2}\right)$ and $\max \left(a_{0}+a_{3}, 2 \pi-a_{1}-a_{2}\right)$, respectively, by varying $a$. Since one of the inequalities becomes an equality for such a point in $L_{2}$, we obtain by Remark 2.7 a degenerate point $P_{0}$ mentioned in Lemma 4.5.1 c. For a similar argument, $t_{3} C=\left[t_{3} P_{0}, b\right]$ or $t_{3} C=\left[t_{3} P_{0}, b\left[\right.\right.$ or $t_{3} C=\left[t_{3} P_{0}, \infty\left[\right.\right.$ for some $t_{3} P_{0}<b$. In the last case, we visualize $C$ as a topological plane and $L_{2}$, as a topological line dividing $C$ into 2 parts.

Now we may accomplish the case c. By Remark 2.10, there exist geodesics $\gamma, \gamma_{0}, \gamma_{1}, \gamma_{2}$ such that $\gamma_{1}, \gamma_{2}$ are tangent, $\alpha\left(\gamma_{0}, \gamma_{1}\right)=a_{0}, \alpha\left(\gamma_{1}, \gamma\right)=a_{1}, \alpha\left(\gamma, \gamma_{2}\right)=a_{2}$, and $\alpha\left(\gamma_{2}, \gamma_{0}\right)=a_{3}$ (for the second type, $\alpha\left(\gamma_{0}, \gamma_{2}\right)=a_{3}, \alpha\left(\gamma_{2}, \gamma\right)=a_{2}, \alpha\left(\gamma, \gamma_{1}\right)=a_{1}$, and $\left.\alpha\left(\gamma_{1}, \gamma_{0}\right)=a_{0}\right)$. Denote by $I, I_{0}, I_{1}, I_{2}$ the reflections in $\gamma, \gamma_{0}, \gamma_{1}, \gamma_{2}$. By Remark 2.3, we obtain elliptic isometries $R_{0}:=I_{1} I_{0}, R_{1}:=I I_{1}, R_{2}:=I_{2} I, R_{3}:=I_{0} I_{2}$ (elliptic isometries $R_{0}:=I_{0} I_{1}, R_{1}:=I_{1} I, R_{2}:=I I_{2}, R_{3}:=I_{2} I_{0}$ for the second type) of the conjugacy classes $R_{c, k_{0}}, R_{c, k_{1}}, R_{c, k_{2}}, R_{c, k_{3}}$ subject to $R_{2} R_{1} R_{0} R_{3}=1$ (to $R_{1} R_{2} R_{3} R_{0}=1$ for the second type) with parabolic $R$. So, we have constructed a point $P \in S$ with parabolic $R$. This point belongs to some component $C$. Hence, there is a regular point $P^{\prime} \in L_{2} \subset C$ with elliptic $R$. As we saw earlier, $C$ should contain the degenerate point $P_{0}$ considered above. Therefore, such a component (of a given type) is unique and we are done in the case $\mathbf{c}$ because we already know that the presence of a point $P \in L_{2}$ with hyperbolic $R$ (induced by the presence of a point with parabolic $R$ ) implies $] t_{0}, \infty\left[\subset t_{3} C\right.$.

By Remarks 2.6, 2.4, and 2.3, in an arbitrary case $\mathbf{a}-\mathbf{e}$, we get in a similar way a point $P_{t} \in S$ of a given type, depending smoothly on $t>t_{0}$, with hyperbolic $R$ such that $t_{3} P_{t}=t$. This point generates a connected component $C$ of $S$ such that $P_{t} \in L_{2}$ and we already know that $\left.t_{3} C \supset\right] t_{0}, \infty[$. Hence, by the uniqueness in Remark 2.6, there exists a unique connected component $C$ of $S$ (of a given type) containing a point with hyperbolic $R$. In the case $\mathbf{c}$, such a component was already constructed above. In the case $\mathbf{d}$, we obtain $t_{3} C=\left[t_{0}, \infty\right.$ [ by Lemma 4.5.1. In all the other cases, we have $\left.t_{3} C=\right] t_{0}, \infty$ [ because $t_{0} \in t_{3} C$ would imply the existence of a point with parabolic $R$ and we have already seen that this is possible only in the case c. Moreover, as $P_{t}$ depends smoothly on $t>t_{0}$, the curve $L_{2}$ gets a smooth parameterization by $t_{3}$ at the points with hyperbolic $R$.

We have now accomplished the cases $\mathbf{b}$ and $\mathbf{e}$. Indeed, in these cases, there cannot exist a regular point $P \in L_{2}$ with parabolic or elliptic $R$ since this is possible only in the cases $\mathbf{c}$ and $\mathbf{a}$, respectively. As $t_{3} P^{\prime}<t_{0}$ for any point $P^{\prime} \in S$ degenerate with respect to $t_{3}$, we conclude that, in the case $\mathbf{b}$, 
there exist a unique connected component $C$ of $\left.S, t_{3} C=\right] t_{0}, \infty[$, whose points have hyperbolic $R$ and a unique component consisting of a single degenerate point. Similarly, in the case e, we get a unique connected component $C$ of $\left.S, t_{3} C=\right] t_{0}, \infty[$, whose points have hyperbolic $R$. Thus, in the cases $\mathbf{b}$ and $\mathbf{e}$, we visualize the unique connected component $C$ of $S$ containing points with hyperbolic $R$ as a topological plane and $L_{2}$, as a topological line dividing $C$ into 2 parts.

There is a unique connected component $C,] 1, \infty\left[\subset t_{3} C\right.$, in the case $\mathbf{d}$. By Lemma 4.5.1, $t_{3} C=[1, \infty[$. Clearly, $\left(t_{1} P, t_{2} P, t_{3} P\right)$ tends to $\left(t_{1}, t_{2}, 1\right)$ with $t_{1}=t_{2}$ when a point $P \in C$ tends to a point boundary with respect to $t_{3}$ because $c_{0}=c_{3}$ and $c_{1}=c_{2}$ for a boundary point. Given $\left.t \in\right] 1, \infty[$, there exists a unique point $P_{t} \in L_{2}$, depending continuously on $t$, such that $t_{3} P_{t}=t$. Any point $P \in C$ with $t_{3} P=t$ has the form $P=g P_{t}$ for a suitable (unique) isometry $g \in C_{R}$. Note that, for $t$ close to 1 , we have $t_{1}\left(g P_{t}\right)$ close to $t_{1}\left(g^{-1} P_{t}\right)$ just because $c_{0}$ and $c_{1}$ are close for $P_{t}$. This means that the points $g P_{t}$ and $g^{-1} P_{t}$ tend to a same boundary point when $g P_{t}$ tends to a boundary point. In other words, in the fibre over $t_{3}=1$, two distinct points over $t>1$ tend to collide when $t$ tends to 1 (unless $g$ tends to 1 ). So, in the case $\mathbf{d}$, we visualize $C$ as a topological plane and $L_{2}$, as a topological line dividing $C$ into 2 parts.

Finally, in the case a, it suffices to construct a regular point $P \in S$ with elliptic $R$ because, as we have seen above, such a point $P$ provides the two degenerate points $P_{1}$ and $P_{2}$ in the connected component containing $P$, implying the existence and uniqueness of a connected component of $S$ whose points have elliptic $R$.

By Remark 2.8, there exist geodesics $\gamma, \gamma_{0}, \gamma_{1}, \gamma_{2}$ such that $\gamma_{1}, \gamma_{2}$ intersect, $\alpha\left(\gamma_{0}, \gamma_{1}\right)=a_{0}, \alpha\left(\gamma_{1}, \gamma\right)$ $=a_{1}, \alpha\left(\gamma, \gamma_{2}\right)=a_{2}$, and $\alpha\left(\gamma_{2}, \gamma_{0}\right)=a_{3}$ (for the second type, $\alpha\left(\gamma_{0}, \gamma_{2}\right)=a_{3}, \alpha\left(\gamma_{2}, \gamma\right)=a_{2}, \alpha\left(\gamma, \gamma_{1}\right)=a_{1}$, and $\left.\alpha\left(\gamma_{1}, \gamma_{0}\right)=a_{0}\right)$. Denote by $I, I_{0}, I_{1}, I_{2}$ the reflection in $\gamma, \gamma_{0}, \gamma_{1}, \gamma_{2}$. By Remark 2.3 , the isometries $R_{0}:=I_{1} I_{0}, R_{1}:=I I_{1}, R_{2}:=I_{2} I, R_{3}:=I_{0} I_{2}$ (the isometries $R_{0}:=I_{0} I_{1}, R_{1}:=I_{1} I, R_{2}:=I I_{2}, R_{3}:=$ $I_{2} I_{0}$ for the second type) of the conjugacy classes $R_{c, k_{0}}, R_{c, k_{1}}, R_{c, k_{2}}, R_{c, k_{3}}$ subject to $R_{2} R_{1} R_{0} R_{3}=1$ (to $R_{1} R_{2} R_{3} R_{0}=1$ for the second type) provide a regular point $P \in S$ with elliptic $R$

For given $\left.a_{0}, a_{1}, a_{2}, a_{3} \in\right] 0, \pi\left[\right.$, we denote $k_{j}:=e^{a_{j} i} \neq \pm 1$ and $u_{j}:=t_{j}\left(\bar{k}_{j}-k_{j}\right)+k_{j}$.

Let $C \subset S:=S\left(a_{0}, a_{1}, a_{2}, a_{3}\right)$ be a connected component of $S$ and let $P \in S$. Then, placing the label $c_{0}$ at $p_{3}$, the relation $R_{c_{3}, k_{3}} R_{c_{2}, k_{2}} R_{c_{1}, k_{1}} R_{c_{0}, k_{0}}=1$ holds in $\mathrm{PU} V$ if $P$ is of the first type and the relation $R_{c_{1}, k_{1}} R_{c_{2}, k_{2}} R_{c_{3}, k_{3}} R_{c_{0}, k_{0}}=1$ holds in PU $V$ if $P$ is of the second type. At the level of SU $V$, we obtain respectively the relations $R_{c_{3}, k_{3}} R_{c_{2}, k_{2}} R_{c_{1}, k_{1}} R_{c_{0}, k_{0}}= \pm 1$ and $R_{c_{1}, k_{1}} R_{c_{2}, k_{2}} R_{c_{3}, k_{3}} R_{c_{0}, k_{0}}= \pm 1$ in $\mathrm{SU} V$. They can be rewritten as the relations $R_{c_{3}, k_{3}} R_{c_{2}, k_{2}} R_{c_{1}, k_{1}} R_{c_{0}, \pm k_{0}}=1$ and $R_{c_{1}, k_{1}} R_{c_{2}, k_{2}} R_{c_{3}, k_{3}} R_{c_{0}, \pm k_{0}}=1$ in SU $V$. By Lemma 3.1, we arrive at the equation (3.5) (when $\pm k_{0}=k_{0}$ ) or at the equation (3.7) (when $\pm k_{0}=-k_{0}$ ) independently of the type of $P$. This means that $C$ satisfies the equation (3.5) or the equation (3.7).

The following corollary claims the converse, i.e., that the components of $S$ are given by the type of hexagons and by one of the equations (3.5) and (3.7).

4.5.3. Corollary. Let $\left.a_{0}, a_{1}, a_{2}, a_{3} \in\right] 0, \pi\left[\right.$. Denote $k_{j}:=e^{a_{j} i}$ and $u_{j}:=t_{j}\left(\bar{k}_{j}-k_{j}\right)+k_{j}$.

For any type of hexagons, the solutions of the equation (3.5) in $t_{1}, t_{2}, t_{3} \geqslant 1$ form a connected component $C_{1}$ of $S:=S\left(a_{0}, a_{1}, a_{2}, a_{3}\right)$ of this type, topologically a plane, with hyperbolic isometry $R_{0} R_{3}$ for some point in the component.

The equation (3.7) in $t_{1}, t_{2}, t_{3} \geqslant 1$ has no solutions in the cases $\mathbf{c}-\mathbf{e}$ of Lemma 4.5.1. In the case $\mathbf{b}$, the equation (3.7) in $t_{1}, t_{2}, t_{3} \geqslant 1$ has a unique solution that constitutes a connected component $C_{0}$ of $S$ of any given type and the isometry $R_{0} R_{3}$ is elliptic for this point. In the case a, the solutions of the equation (3.7) in $t_{1}, t_{2}, t_{3} \geqslant 1$ form a connected component $C_{0}$ of $S$ (of either type), topologically a 2 -sphere, and the isometry $R_{0} R_{3}$ is elliptic for every point in this component.

The above is a full list of connected components of $S$.

Proof. Let us first observe that a point $P \in S$ degenerate with respect to $t_{3}$ does not satisfy the equation (3.5) in the cases $\mathbf{a}-\mathbf{b}$. Indeed, if $t_{3} P=1$, this assertion is just Lemma 3.4. If $c_{1}=c_{2}$, 
we can treat the point $P$ as a point in the space $S^{\prime}:=S\left(a_{2}, a_{3}, a_{0}, a_{1}\right)$ : if $P$ corresponds to the relation $R_{3} R_{2} R_{1} R_{0}=1$ (to the relation $R_{0} R_{1} R_{2} R_{3}=1$ for the second type), then $P^{\prime}$ corresponds to the relation $R_{1} R_{0} R_{3} R_{2}=1$ (to the relation $R_{2} R_{3} R_{0} R_{1}=1$ for the second type), and the assertion follows from Lemma 3.4 applied to $S^{\prime}$, where $t_{3} P^{\prime}$ becomes 1 , because the equation (3.5) and the cases $\mathbf{a}-\mathbf{b}$ remain the same for $S^{\prime}$.

In an arbitrary case a-e, there exists a unique connected component $C_{1}$ of $S$ (of either type) possessing a point with hyperbolic $R_{0} R_{3}$. Such a component $C_{1}$ cannot be compact since $] t_{0}, \infty\left[\subset t_{3} C_{1}\right.$. By Lemma 3.6, $C_{1}$ has to satisfy the equation (3.5). By Proposition 4.5.2, there is a unique connected component (of either type) in the cases $\mathbf{c}-\mathbf{e}$; so, we are done in these cases.

In the remaining cases $\mathbf{a}-\mathbf{b}$, there are two connected components $C_{0}, C_{1}$ (of either type) and $C_{0}$, the compact one, does not satisfy the equation (3.5) because, by Lemma 4.5.1, it contains a point $P$ degenerate with respect to $t_{3}$. On the other hand, the component $C_{1}$ containing a point with hyperbolic $R_{0} R_{3}$ is not compact because $] t_{0}, \infty\left[\subset t_{3} C_{1}\right.$, hence, $C_{1}$ cannot satisfy the equation (3.7) by Lemma 3.6. Consequently, $C_{0}$ satisfies (3.7) and $C_{1}$ satisfies (3.5) and we are done

We already know (see the second paragraph in 4.5) that the group $M_{3}$ is generated by the modifications $n_{1}, n_{2}, n_{3}$ and that $n_{j}$ alters the type of a hexagon and keeps $t_{l}$ for $l \neq j$. Let us write the equations (3.5) and (3.7) in the form $e_{1}\left(t_{1}, t_{2}, t_{3}\right)=0$ and $e_{0}\left(t_{1}, t_{2}, t_{3}\right)=0$, respectively. Since $n_{j}$ transforms the relation $R_{c_{3}, k_{3}} R_{c_{2}, k_{2}} R_{c_{1}, k_{1}} R_{c_{0}, k_{0}}=1$ in $\mathrm{SU} V$ into the relation $R_{c_{1}, k_{1}} R_{c_{2}, k_{2}} R_{c_{3}, k_{3}} R_{c_{0}, k_{0}}=1$ in SU $V$ and vice-versa (analogously, for the relation $R_{c_{3}, k_{3}} R_{c_{2}, k_{2}} R_{c_{1}, k_{1}} R_{c_{0},-k_{0}}=1$ in SUV), this modification preserves the equations $e_{l}\left(t_{1}, t_{2}, t_{3}\right)=0$ by Lemma 3.1. It is easy to see that $e_{l}\left(t_{1}, t_{2}, t_{3}\right)=$ $4 t_{j}^{2} \sin ^{2} a_{j}+$ lower terms in $t_{j}$ with $\sin a_{j} \neq 0$, i.e., the equation $e_{l}\left(t_{1}, t_{2}, t_{3}\right)=0$ is quadratic in $t_{j}$. Therefore, at the level of the $t_{k}$ 's, the modification $n_{j}$ simply interchanges the roots of $e_{l}\left(t_{1}, t_{2}, t_{3}\right)=0$ in $t_{j}$. In what follows, we may not distinguish anymore the types of hexagons and we may identify the hexagons of different types with the same $\left(t_{1}, t_{2}, t_{3}\right)$. In this way, $n_{j}$ acts as an involution on every component $C_{l}$ of $S$, where $C_{l}$ is now given by the equation $e_{l}\left(t_{1}, t_{2}, t_{3}\right)=0$. (Perhaps, it would be more correct to pass to the index 2 subgroup $M$ of the group $M_{3}$. However, it is more convenient to deal with the involutions and this makes no difference in the problem of the discreteness of the action of $M_{3}$ on $S$. )

4.5.4. Definition. Let $1 \leqslant j \leqslant 3$. Denote by $A_{j} \subset S:=S\left(a_{0}, a_{1}, a_{2}, a_{3}\right)$ the set of fixed points of the involution $n_{j}$. By the above, in the connected component $C_{l}$ of $S$ given by the equation $e_{l}\left(t_{1}, t_{2}, t_{3}\right)=0$, the set $A_{j}$ is given by the equation $\frac{\partial}{\partial t_{j}} e_{l}\left(t_{1}, t_{2}, t_{3}\right)=0$. In particular, $A_{1} \cap A_{2} \cap A_{3}$ is the set of all singular points of $S$.

In the proof of Proposition 4.5.2, placing the label $c_{0}$ at $p_{3}$, we defined a curve $L_{2} \subset S$ of all points $P \in S$ degenerate or boundary with respect to $t_{3}$ and of all those points $P \in S$ regular with respect to $t_{3}$ that are subject to $R_{0}=I_{1} I_{0}, R_{1}=I I_{1}, R_{2}=I_{2} I, R_{3}=I_{0} I_{2}$ when $P$ is of the first type and are subject to $R_{0}=I_{0} I_{1}, R_{1}=I_{1} I, R_{2}=I I_{2}, R_{3}=I_{2} I_{0}$ when $P$ is of the second type, where $I, I_{0}, I_{1}, I_{2}$ are suitable reflections in geodesics.

4.5.5. Lemma. $A_{2}=L_{2}$.

Proof. If $P \in S$ is degenerate with respect to $t_{3}$, then $P$ is clearly a fixed point of $n_{2}$ because $P$ is a unique point in $S$ with the value $t_{3} P$ of $t_{3}$ and $n_{2}$ keeps the values of $t_{3}$.

Definition 4.5.4 says that $P \in A_{2}$ iff $\operatorname{ta}\left(c_{2}, p_{2}\right)=\operatorname{ta}\left(p_{3}, c_{2}\right)$ for $P$. For a point $P \in S$ boundary with respect to $t_{3}$, this condition is empty since $p_{2}=p_{3}$ in this case.

So, we assume $P \in S$ to be regular with respect to $t_{3}$. Then $c_{0} \neq c_{3}$ (we place the label $c_{0}$ at $p_{3}$ ) and $c_{1} \neq c_{2}$.

Suppose that $P \in L_{2}$. Then $R_{1}=I I_{1}$ if $P$ is of the first type and $R_{1}=I_{1} I$ if $P$ is of the second type, where $I c_{2}=c_{2}, p_{1}=R_{1} p_{3}=I I_{1} c_{0}=I c_{0}$ for $P$ of the first type, and $p_{1}=R_{1}^{-1} p_{3}=I I_{1} c_{0}=I c_{0}$ for $P$ of the second type. Therefore, $\operatorname{ta}\left(c_{2}, p_{2}\right)=\operatorname{ta}\left(p_{1}, c_{2}\right)=\operatorname{ta}\left(I c_{0}, I c_{2}\right)=\operatorname{ta}\left(p_{3}, c_{2}\right)$, i.e., $P \in A_{2}$. 
Conversely, let $P \in A_{2}$, i.e., ta $\left(c_{2}, p_{2}\right)=\operatorname{ta}\left(p_{3}, c_{2}\right)$. Denote by $I$ and $I_{0}$ the reflections in the geodesics joining $c_{1}, c_{2}$ and $c_{0}, c_{3}$, respectively. Since $\operatorname{ta}\left(c_{0}, c_{1}\right)=\operatorname{ta}\left(c_{1}, p_{1}\right)$ and $\operatorname{ta}\left(p_{1}, c_{2}\right)=\operatorname{ta}\left(c_{2}, p_{2}\right)=\operatorname{ta}\left(c_{0}, c_{2}\right)$, the triangles $\left(c_{1}, c_{0}, c_{2}\right)$ and $\left(c_{1}, p_{1}, c_{2}\right)$ are congruent. As $p_{1}=c_{0}$ implies $c_{0}=c_{1}=p_{1}$ due to $p_{1}=R_{1} c_{0}$ (when $P$ is of the first type) or $c_{0}=R_{1} p_{1}$ (when $P$ is of the second type), we obtain $p_{1}=I c_{0}$.

Suppose that $c_{2} \neq c_{3}$ and denote by $I_{2}$ the reflection in the geodesic joining $c_{2}$ and $c_{3}$. Since $\operatorname{ta}\left(c_{2}, p_{2}\right)=\operatorname{ta}\left(c_{0}, c_{2}\right)$ and $\operatorname{ta}\left(p_{2}, c_{3}\right)=\operatorname{ta}\left(c_{3}, c_{0}\right)$, the triangles $\left(c_{2}, p_{2}, c_{3}\right)$ and $\left(c_{2}, c_{0}, c_{3}\right)$ are congruent. As $c_{0}=R_{3} p_{2}$ or $p_{2}=R_{3} c_{0}$, the equality $p_{2}=c_{0}$ would imply $p_{2}=c_{0}=c_{3}$. Hence, $p_{2}=I_{2} c_{0}$ and $p_{2} \neq c_{2}$ because $p_{2}=c_{2}$ and $\operatorname{ta}\left(c_{2}, p_{2}\right)=\operatorname{ta}\left(c_{0}, c_{2}\right)$ would imply $p_{2}=c_{0}$.

Let $P$ be of the first type. Then $R_{3} p_{2}=c_{0}, R_{3} c_{3}=c_{3}, I_{0} I_{2} p_{2}=I_{0} I_{2} I_{2} c_{0}=c_{0}, I_{0} I_{2} c_{3}=c_{3}$, $R_{2} p_{1}=p_{2}, R_{2} c_{2}=c_{2}, I_{2} I p_{1}=I_{2} I I c_{0}=I_{2} c_{0}=p_{2}, I_{2} I c_{2}=c_{2}$ with $c_{0} \neq c_{3}$ and $p_{2} \neq c_{2}$. Hence, $R_{3}=I_{0} I_{2}$ and $R_{2}=I_{2} I$. Writing $R_{0}$ as $R_{0}=I_{1} I_{0}$, where $I_{1}$ is a suitable reflection in a geodesic, we conclude from the relation $R_{3} R_{2} R_{1} R_{0}=1$ that $R_{1}=I I_{1}$. Therefore, $P \in L_{2}$.

Let $P$ be of the second type. Then $R_{3} c_{0}=p_{2}, R_{3} c_{3}=c_{3}, I_{2} I_{0} c_{0}=I_{2} c_{0}=p_{2}, I_{2} I_{0} c_{3}=c_{3}, R_{2} p_{2}=p_{1}$, $R_{2} c_{2}=c_{2}, I I_{2} p_{2}=I I_{2} I_{2} c_{0}=I c_{0}=p_{1}, I I_{2} c_{2}=c_{2}$ with $c_{0} \neq c_{3}$ and $p_{2} \neq c_{2}$. Hence, $R_{3}=I_{2} I_{0}$ and $R_{2}=I I_{2}$. Writing $R_{0}$ as $R_{0}=I_{0} I_{1}$, where $I_{1}$ is a suitable reflection in a geodesic, we conclude from the relation $R_{1} R_{2} R_{3} R_{0}=1$ that $R_{1}=I_{1} I$. Therefore, $P \in L_{2}$.

Suppose that $c_{2}=c_{3}$ and $c_{0} \neq c_{1}$. Denote by $I_{1}$ the geodesic joining $c_{0}$ and $c_{1}$.

If $P$ is of the first type, then $R_{1}=I I_{1}$ because $R_{1} c_{0}=p_{1}, R_{1} c_{1}=c_{1}, I I_{1} c_{0}=I c_{0}=p_{1}, I I_{1} c_{1}=c_{1}$ with $c_{0} \neq c_{1}$. We have $R_{2}=I_{2} I$ and $R_{3}=I_{0}^{\prime} I_{2}$, where $I_{2}$ and $I_{0}^{\prime}$ are suitable reflections in geodesics. From the relation $R_{3} R_{2} R_{1} R_{0}=1$, we obtain $R_{0}=I_{1} I_{0}^{\prime}$, hence, $I_{0}^{\prime} c_{0}=c_{0}$. Since $c_{0} \neq c_{3}$ and $I_{0}^{\prime} c_{3}=c_{3}$, we conclude that $I_{0}^{\prime}=I_{0}$. Therefore, $P \in L_{2}$.

If $P$ is of the second type, then $R_{1}=I_{1} I$ because $R_{1} p_{1}=c_{0}, R_{1} c_{1}=c_{1}, I_{1} I p_{1}=I_{1} I I c_{0}=c_{0}$, $I_{1} I c_{1}=c_{1}$ with $c_{0} \neq c_{1}$. We have $R_{2}=I I_{2}$ and $R_{3}=I_{2} I_{0}^{\prime}$, where $I_{2}$ and $I_{0}^{\prime}$ are suitable reflections in geodesics. From the relation $R_{1} R_{2} R_{3} R_{0}=1$, we obtain $R_{0}=I_{0}^{\prime} I_{1}$, hence, $I_{0}^{\prime} c_{0}=c_{0}$. Since $c_{0} \neq c_{3}$ and $I_{0}^{\prime} c_{3}=c_{3}$, we conclude that $I_{0}^{\prime}=I_{0}$. Therefore, $P \in L_{2}$.

Finally, suppose that $c_{0}=c_{1}$ and $c_{2}=c_{3}$. Then $I=I_{0}$. Since $c_{0} \neq c_{3}$, we get $R_{1} R_{0}=1$ and $R_{3} R_{2}=1$. Since $R_{0}=I_{1} I_{0}, R_{3}=I_{0} I_{2}$ for $P$ of the first type and $R_{0}=I_{0} I_{1}, R_{3}=I_{2} I_{0}$ for $P$ of the second type, where $I_{1}$ and $I_{2}$ are suitable reflections in geodesics, we arrive at $R_{1}=I_{0} I_{1}, R_{2}=I_{2} I_{0}$ and at $R_{1}=I_{1} I_{0}, R_{2}=I_{0} I_{2}$, respectively. Therefore, $P \in L_{2}$

4.5.6. Lemma. A point $P \in S:=S\left(a_{0}, a_{1}, a_{2}, a_{3}\right)$ belongs to $A_{1} \cap A_{2}$ iff $P$ is degenerate or boundary with respect to $t_{3}$.

A point $P \in S$ is singular iff $c_{1}=p_{1}=c_{2}=p_{2}=c_{3}=p_{3}$ for $P$. Therefore, $S$ is not smooth iff $\sum_{j} a_{j}=2 \pi$. In this case, there exists a unique singular point in $S$; it belongs to the noncompact component $C_{1}$.

Proof. We observe first that there exists at most one point equidistant from 3 pairwise distinct points. Clearly, $P \in A_{j}$ iff $c_{j}$ is equidistant from $p_{1}, p_{2}, p_{3}$.

Let $P \in A_{1} \cap A_{2}$. If the points $p_{1}, p_{2}, p_{3}$ are pairwise distinct, then $c_{1}=c_{2}$. If $p_{1}=p_{2}$, then $p_{1}=c_{2}=p_{2}$, hence, $p_{3}=c_{2}$, therefore, $c_{1}=p_{1}=c_{2}$ again. If $p_{1}=p_{3}$, then $p_{1}=c_{1}=p_{3}$, hence, $p_{2}=c_{1}$, therefore, $c_{1}=c_{2}$ again. If $p_{2}=p_{3}$, then $c_{3}=p_{3}$. So, $P$ is degenerate or boundary with respect to $t_{3}$. It is easy to see that the converse is also true: if $c_{1}=c_{2}$ or $c_{3}=p_{3}$ for $P$, then $P \in A_{1} \cap A_{2}$.

We know now that $P$ is singular iff $c_{1}=c_{2}$ or $c_{3}=p_{3}$ and, simultaneously, $c_{2}=c_{3}$ or $c_{1}=p_{1}$. If $c_{1}=c_{2}=c_{3}$, then, placing the label $c_{0}$ at $p_{3}$, it follows from $R_{3} R_{2} R_{1} R_{0}=1$ or from $R_{1} R_{2} R_{3} R_{0}=1$ that $c_{0}=c_{1}$ because $R_{0} \neq 1$. Therefore, $c_{1}=p_{1}=c_{2}=p_{2}=c_{3}=p_{3}$. If $c_{1}=p_{1}=c_{2}\left(\right.$ or $\left.c_{2}=c_{3}=p_{3}\right)$, then $p_{3}=c_{1}$ (respectively, $p_{2}=c_{3}$ ) and, again, $c_{1}=p_{1}=c_{2}=p_{2}=c_{3}=p_{3}$. If $c_{1}=p_{1}$ and $c_{3}=p_{3}$, then $p_{3}=c_{1}$ and $p_{2}=c_{3}$, implying $c_{1}=p_{1}=c_{2}=p_{2}=c_{3}=p_{3}$ because $R_{2}$ has a unique fixed point

4.6. Discreteness of $M_{3}$. Placing the label $c_{0}$ at $p_{3}$, an arbitrary hexagon $P \in S\left(a_{0}, a_{1}, a_{2}, a_{3}\right)$ of the first type corresponds to a relation $R_{3} R_{2} R_{1} R_{0}=1$. It is easy to see that, the hexagon $P n_{2} n_{1}$ corresponds 
to the relation $R_{3} R_{2}^{\left(R_{1}^{R_{2}}\right)} R_{1}^{R_{2}} R_{0}=1$, i.e, to the relation $R_{3} R_{2}^{R_{2} R_{1}} R_{1}^{R_{2} R_{1}} R_{0}=1$. By induction on $l$, $P\left(n_{2} n_{1}\right)^{l}$ corresponds to the relation $R_{3} R_{2}^{\left(R_{2} R_{1}\right)^{l}} R_{1}^{\left(R_{2} R_{1}\right)^{l}} R_{0}=1$. Since $R_{0} R_{3}=\left(R_{2} R_{1}\right)^{-1}$, the action of $n_{2} n_{1}$ preserves the fibres of $S\left(a_{0}, a_{1}, a_{2}, a_{3}\right) \stackrel{t_{3}}{\longrightarrow} \mathbb{R}$.

In particular, if $P$ is regular with respect to $t_{3}$ with elliptic $R_{0} R_{3}$ and the action of $M_{3}$ on the connected component containing $P$ is discrete, then $R_{0} R_{3}$ has to be periodic and the period has to be the same when we slightly vary $t_{3} P$. This is impossible because the triangle $\left(c_{0}, c, c_{3}\right)$ that corresponds to the relation $R_{0}^{-1}\left(R_{0} R_{3}\right) R_{3}^{-1}=1$ (see, for instance, the proof of Lemma 4.4.1) is completely determined by its angles and, hence, does not admit any variation of $t_{3}$, where $c$ stands for the fixed point of $R_{0} R_{3}$. Consequently, the group $M$ cannot act discretely on a component containing a point regular with respect to $t_{3}$ with elliptic $R_{0} R_{3}$. (We denote by $M$ the index 2 subgroup in the group $M_{3}$.)

4.6.1. Lemma. If $M$ act discretely on a connected component of $S\left(a_{0}, a_{1}, a_{2}, a_{3}\right)$, then the component is the noncompact one (we drop the component of a single point) and

- $a_{j}+a_{k} \leqslant \pi$ for all $j \neq k$ or $\pi \leqslant a_{j}+a_{k}$ for all $j \neq k$.

Proof. Suppose that $a_{j}+a_{k}<\pi<a_{l}+a_{m}$ with $j \neq k$ and $l \neq m$. Assuming without loss of generality that $a_{0} \leqslant a_{3} \leqslant a_{1} \leqslant a_{2}$, we obtain $a_{0}+a_{3}<\pi<a_{1}+a_{2}$, i.e., we are in the case $\mathbf{c}$. By Proposition 4.5.2, we get a unique component. It possesses a point regular with respect to $t_{3}$ with elliptic $R_{0} R_{3}$. As we saw above, $M$ cannot act discretely on this component

In the sequel, we deal only with the cases listed in Lemma 4.6.1 and with the noncompact connected component $C_{1}$.

By Lemma 4.5.6, the component $C_{1}$ is not smooth only in the case $a_{0}=a_{1}=a_{2}=a_{3}=\frac{\pi}{2}$. Since, by Lemma 4.5.6, $A_{1} \cap A_{2} \cap C_{1} \neq \varnothing$ only in the case $\mathbf{d}$ of Lemma 4.5.1, the curves $A_{j}$ are pairwise disjoint in $C_{1}$ if $C_{1}$ is smooth.

For $1 \leqslant j \leqslant 3$ and $P \in S\left(a_{0}, a_{1}, a_{2}, a_{3}\right)$, denote $t_{j}^{\prime} P:=\operatorname{ta}\left(c_{j}, p_{j+1}\right)$ (the indices are modulo 3$)$. So, the condition $t_{j} P=t_{j}^{\prime} P$ is equivalent to $P \in A_{j}$. By Proposition 4.5.2 and Lemma 4.5.5, assuming $C_{1}$ smooth, the curve $A_{j}$ is a smooth line in $C_{1}$ and divides the smooth plane $C_{1}$ into two parts $H_{j}$ and $H_{j}^{\prime}$ given by the inequalities $t_{j} \leqslant t_{j}^{\prime}$ and $t_{j} \geqslant t_{j}^{\prime}$, respectively.

4.6.2. Lemma. Suppose that

- $a_{j}+a_{k} \leqslant \pi$ for all $j \neq k$ or $\pi \leqslant a_{j}+a_{k}$ for all $j \neq k$.

Then the noncompact connected component $C_{1}$ of $S\left(a_{0}, a_{1}, a_{2}, a_{3}\right)$, i.e., the one given by the equation $e_{1}\left(t_{1}, t_{2}, t_{3}\right)=0$, is smooth unless $a_{0}=a_{1}=a_{2}=a_{3}=\frac{\pi}{2}$. Assuming $C_{1}$ smooth, the curves $A_{1}, A_{2}, A_{3}$ in $C_{1}$ are smooth pairwise disjoint lines, each divides the smooth plane $C_{1}$ into two parts, and, moreover, $A_{k} \cap C_{1} \subset H_{j} \cap C_{1}$ for all $j$ and $k$.

Proof. Suppose that, say, $A_{2} \cap C_{1} \not \subset H_{1} \cap C_{1}$. Note that the inequalities $0<a_{j}<\pi$ and $a_{j}+a_{k} \leqslant \pi$ for all $j \neq k$ define in $\mathbb{R}^{4}$ a convex region that remains connected after removing the point $\left(\frac{\pi}{2}, \frac{\pi}{2}, \frac{\pi}{2}, \frac{\pi}{2}\right)$ (similarly, for the inequalities $\pi \leqslant a_{j}+a_{k}$ ). Thus, we can vary the $a_{j}$ 's keeping $C_{1}$ smooth. Since $A_{1} \cap A_{2} \cap C_{1}=\varnothing$ during the deformation and the equation $e_{1}\left(t_{1}, t_{2}, t_{3}\right)=0$ as well as the functions $t_{1}^{\prime}, t_{2}^{\prime}$ depend continuously on the $a_{j}$ 's, we obtain $A_{2} \cap C_{1} \not \subset H_{1} \cap C_{1}$ for the special case of $a_{1}=a_{2}=a_{3}=\frac{\pi}{2}$ and $\left.a_{0} \in\right] 0, \frac{\pi}{2}\left[\right.$. In this case, a hexagon are just a triangle with the vertices $p_{1}, p_{2}, p_{3}$ whose interior angles sum to $2 a_{0}$, and $c_{1}, c_{2}, c_{3}$ are respectively the middle points of the sides $\left[p_{3}, p_{1}\right],\left[p_{1}, p_{2}\right],\left[p_{2}, p_{3}\right]$ of the triangle. As $A_{2} \cap C_{1} \not \subset H_{1} \cap C_{1}$ implies $A_{2} \cap H_{1} \cap C_{1}=\varnothing$, the isosceles triangle $\left(p_{1}, p_{2}, p_{3}\right)$ with $\operatorname{dist}\left(p_{1}, p_{3}\right)=\operatorname{dist}\left(p_{2}, p_{3}\right)$ and $\operatorname{dist}\left(p_{1}, p_{2}\right)=2 \operatorname{dist}\left(c_{2}, p_{3}\right)$ whose interior angles sum to $2 a_{0}$ satisfies $2 \operatorname{dist}\left(c_{1}, p_{2}\right)<\operatorname{dist}\left(p_{1}, p_{3}\right)$, which is impossible

4.6.3. Theorem. The group $M_{3}$ generated by the modifications acts discretely on a component $C$ of $S\left(a_{0}, a_{1}, a_{2}, a_{3}\right)$ iff $C$ is noncompact, 
- $a_{j}+a_{k} \leqslant \pi$ for all $j \neq k$ or $\pi \leqslant a_{j}+a_{k}$ for all $j \neq k$, and $a_{j} \neq \frac{\pi}{2}$ for some $0 \leqslant j \leqslant 3$. In this case, the quotient $C / M$ is a 3-holed 2 -sphere and $M$ is a free group of rank 2, where $M$ stands for the index 2 subgroup in $M_{3}$.

Proof. Suppose that $a_{j} \neq \frac{\pi}{2}$ for some $0 \leqslant j \leqslant 3$. By Lemma 4.6.1, it suffices to show that $M_{3}$ acts discretely on the noncompact component $C_{1}$ in the cases mentioned in the theorem.

Denote $T:=H_{1} \cap H_{2} \cap H_{3}$. By Lemma 4.6.2 and Proposition 4.5.2, the copies $T n_{1}, T n_{2}, T n_{3}$ are pairwise disjoint and intersect $T$ only in the lines $A_{1}, A_{2}, A_{3}$, respectively. Moreover, $A_{j}$ lies in the interior of $T_{1}:=T \cup T n_{1} \cup T n_{2} \cup T n_{3}$ and $T_{1}$ is closed, connected, and limited by the pairwise disjoint lines $A_{3} n_{1}, A_{2} n_{1}, A_{1} n_{2}, A_{3} n_{2}, A_{2} n_{3}, A_{1} n_{3}$. Applying to $T_{1}$ the involutions $n_{1} n_{3} n_{1}, n_{1} n_{2} n_{1}, n_{2} n_{1} n_{2}$, $n_{2} n_{3} n_{2}, n_{3} n_{2} n_{3}, n_{3} n_{1} n_{3}$ corresponding to these lines, we obtain pairwise disjoint copies of $T_{1}$. They intersect $T_{1}$ only in the listed lines. Denote by $T_{2}$ the union of $T_{1}$ with the listed copies of $T_{1}$, and so on. The standard arguments will show that the group $M_{3}$ acts discretely on $C_{1}$ and that $T$ is its fundamental region if we observe that $D:=\bigcup_{j} T_{j}$ coincides with $C_{1}$.

As $D$ is $M_{3}$-stable and open in $C_{1}$, its boundary $\partial D:=\bar{D} \backslash D$ is $M_{3}$-stable and closed in $\mathbb{R}^{3}$. It suffices to show that $\partial D=\varnothing$.

Suppose that the intersection $D_{r}$ of $\partial D$ with the closed ball of radius $r>1$ centred at the origin in $\mathbb{R}^{3}$ is nonempty. Since $D_{r}$ is compact, there is a point $P \in D_{r}$ with a minimal value of $t_{1} t_{2} t_{3}$. As $T \cap \partial D=\varnothing$, we have, say, $t_{3}^{\prime} P<t_{3} P$. Then $t_{1}\left(P n_{3}\right)=t_{1} P, t_{2}\left(P n_{3}\right)=t_{2} P, t_{3}\left(P n_{3}\right)=t_{3}^{\prime} P<t_{3} P$, and $P n_{3} \in D_{r}$. This contradicts the choice of $P$.

The remaining case $a_{0}=a_{1}=a_{2}=a_{3}=\frac{\pi}{2}$ is considered in the next remark

4.6.4. Remark. The connected component $C$ of $S:=S\left(\frac{\pi}{2}, \frac{\pi}{2}, \frac{\pi}{2}, \frac{\pi}{2}\right)$ with the action of the group $M$ is isomorphic to the space $\mathbb{R}^{2} / \pm 1$ with the natural action of the congruence subgroup $\Gamma_{2}$ in $\mathrm{SL}_{2} \mathbb{Z}$ (known to be generated by $\left[\begin{array}{ll}1 & 2 \\ 0 & 1\end{array}\right]$ and $\left.\left[\begin{array}{ll}1 & 0 \\ 2 & 1\end{array}\right]\right)$. Therefore, almost every $M$-orbit is dense in $C$.

Proof. All vertices of an arbitrary hexagon $P \in S$ lie on a geodesic. Therefore, seeing a hexagon as a triple $\left(c_{1}, c_{2}, c_{3}\right)$, we can interpret it as a triple of real numbers considered up to isometries of the real line. In these terms, $R_{j}: r \mapsto 2 c_{j}-r$. Since the modifications $n_{2} n_{1}$ and $n_{3} n_{1}$ transform the relation $R_{3} R_{2} R_{1} R_{0}=1$ (we place the label $c_{0}$ at $p_{3}$ ) into the relations $R_{3} R_{2}^{R_{2} R_{1}} R_{1}^{R_{2}} R_{0}=1$ and $R_{3} R_{2}^{R_{1} R_{3}} R_{1} R_{0}^{R_{3} R_{1}}=1$, respectively, assuming $c_{3}:=0 \in \mathbb{R}$, we can see that $n_{2} n_{1}:\left(c_{1}, c_{2}\right) \mapsto$ $\left(2 c_{2}-c_{1}, 2 c_{2}-\left(2 c_{1}-c_{2}\right)\right)=\left(-c_{1}+2 c_{2},-2 c_{1}+3 c_{2}\right)$ and $n_{3} n_{1}:\left(c_{1}, c_{2}\right) \mapsto\left(c_{1}, 2 c_{1}+c_{2}\right)$. In other words, we identify $S$ with $\mathbb{R}^{2} / \pm 1$ where $n_{2} n_{1}$ acts as $\left[\begin{array}{ll}-1 & 2 \\ -2 & 3\end{array}\right]$ and $n_{3} n_{1}$, as $\left[\begin{array}{ll}1 & 0 \\ 2 & 1\end{array}\right]$. It remains to observe that $\left[\begin{array}{ll}1 & 0 \\ 1 & 1\end{array}\right]\left[\begin{array}{ll}1 & 2 \\ 0 & 1\end{array}\right]\left[\begin{array}{cc}1 & 0 \\ -1 & 1\end{array}\right]=\left[\begin{array}{ll}-1 & 2 \\ -2 & 3\end{array}\right]$ and $\left[\begin{array}{ll}1 & 0 \\ 1 & 1\end{array}\right]\left[\begin{array}{ll}1 & 0 \\ 2 & 1\end{array}\right]\left[\begin{array}{cc}1 & 0 \\ -1 & 1\end{array}\right]=\left[\begin{array}{ll}1 & 0 \\ 2 & 1\end{array}\right]$.

\section{Hyperbolic 2-spheres with $n+1$ cone singularities and convex $2 n$-gons}

Given $a_{0}, a_{1}, \ldots, a_{n} \in\left[\frac{\pi}{2}, \pi\left[\right.\right.$ such that $\sum_{j} a_{j}>2 \pi$, we deal with the space $C\left(a_{0}, a_{1}, \ldots, a_{n}\right)$ of hyperbolic 2-spheres $\Sigma$ with labeled cone singularities $c_{0}, c_{1}, \ldots, c_{n}$ such that the apex curvature at $c_{j}$ equals $2 a_{j}$ for all $j$; the 2 -spheres are considered up to orientation- and label-preserving isometries.

Let $\Sigma \in C$. We pick geodesic segments $\left[c_{0}, c_{j}\right] \subset \Sigma$ whose pairwise intersections are just $c_{0}$ (say, the shortest ones) and cut $\Sigma$ along these segments. We get a hyperbolic $2 n$-gon $P$ with consecutive vertices $c_{1}, p_{1}, \ldots, c_{n}, p_{n}$ such that the interior angle at $c_{j}$ equals $2 \pi-2 a_{j} \leqslant \pi$ for all $1 \leqslant j \leqslant n$ and the interior angles at the $p_{j}$ 's sum to $2 \pi-2 a_{0} \leqslant \pi$. It follows that $P$ is star-like, hence, it is embeddable by Remark 2.11 into the hyperbolic plane as a convex $2 n$-gon. Applying to $P$ the modification $m_{j, l}$ (see Definition 4.3), we obtain another convex $2 n$-gon $P m_{j, l}$ such that the sphere $\Sigma$ is glued from $P m_{j, l}$.

In this section, we show that $C\left(a_{0}, a_{1}, \ldots, a_{n}\right)=S_{0}\left(a_{0}, a_{1}, \ldots, a_{n}\right) / M_{n}$, where $M_{n}$ is the group generated by the modifications $m_{j, l}$ and $S\left(a_{0}, a_{1}, \ldots, a_{n}\right) \supset S_{0}\left(a_{0}, a_{1}, \ldots, a_{n}\right)$ is the union of $(n-1)$ ! components formed by all convex $2 n$-gons in $S\left(a_{0}, a_{1}, \ldots, a_{n}\right)$. Components in question correspond 
to types of $2 n$-gons (see Subsection 1.2.5 for the definition of the type). The other components of $S\left(a_{0}, a_{1}, \ldots, a_{n}\right)$ are formed by the $2 n$-gons that are nonsimple as closed curves. It is easy to see that such $2 n$-gons can also be characterized as those that 'limit' area different from $2 \sum_{j} a_{j}-(n+4) \pi$ (see [Ana1] for the definition of the area 'limited' by a nonsimple closed curve).

5.1. Theorem. Let $a_{0}, a_{1}, \ldots, a_{n} \in\left[\frac{\pi}{2}, \pi\left[\right.\right.$ be such that $\sum_{j} a_{j}>2 \pi$. Given convex $2 n$-gons $P, P^{\prime} \in$ $S_{0}\left(a_{0}, a_{1}, \ldots, a_{n}\right)$ such that the corresponding 2-spheres $\Sigma, \Sigma^{\prime}$ are isometric (the orientation and the labels are preserved), there exists $m \in M_{n}$ such that $P^{\prime}=P m$.

5.2. We take a $2 n$-gon $P \in S_{0}\left(a_{0}, a_{1}, \ldots, a_{n}\right)$ with consecutive vertices $c_{1}, p_{1}, \ldots, c_{n}, p_{n}$. Without loss of generality, we assume that the interior angles of $P$ at the $p_{j}$ 's sum to $2 \pi-2 a_{0}$ and the interior angle of $P$ at $c_{j}$ equals $2 \pi-2 a_{j}$ for all $1 \leqslant j \leqslant n$. Denote by $D$ the closed disc in the hyperbolic plane limited by $P$, let $\Sigma$ stand for the 2 -sphere glued from $P$, and let $p, c_{1}, \ldots, c_{n} \in \Sigma$ be respectively the cone points of apex curvatures $2 a_{0}, 2 a_{1}, \ldots, 2 a_{n}$. The sides of $P$ (after gluing) provide simple geodesic segments $s_{j} \subset \Sigma$ joining respectively $p$ and $c_{j}, 1 \leqslant j \leqslant n$, with pairwise intersection $p$.

Any $2 n$-gon $P^{\prime} \in S_{0}\left(a_{0}, a_{1}, \ldots, a_{n}\right)$ that produces the same $\Sigma$ after gluing is therefore nothing but simple geodesic segments $s_{j}^{\prime} \subset \Sigma$ joining respectively $p$ and $c_{j}, 1 \leqslant j \leqslant n$, and $p$ is their pairwise intersection. Some of the $s_{j}$ 's can coincide with some of the $s_{j}^{\prime}$. Denote by $0 \leqslant u \leqslant n$ the number of noncoinciding ones. Clearly, $P^{\prime}=P$ if $u=0$.

In terms of the closed disc $D$, an arbitrary segment $s_{j}^{\prime}$ is given by finitely many disjoint geodesic segments $\left[q_{0}, q_{1}^{\prime}\right],\left[q_{1}, q_{2}^{\prime}\right], \ldots,\left[q_{v-1}, q_{v}^{\prime}\right] \subset D$, where $1 \leqslant v, q_{0}=p_{k}$ for some $1 \leqslant k \leqslant n, q_{v}^{\prime}=c_{j}$, the interior of $\left[q_{l}, q_{l+1}^{\prime}\right]$ lives in the interior of $D$ for all $0 \leqslant l \leqslant v-1$ unless $v=1$ and $k=j-1, j$, and, for all $1 \leqslant l \leqslant v-1$, the points $q_{l}^{\prime} \neq q_{l}$ are in the interiors of different sides of $P$ (glued in $\Sigma$ ), symmetric relatively their common vertex $c_{w_{l}}, 1 \leqslant w_{l} \leqslant n$, i.e., $\operatorname{dist}\left(q_{l}^{\prime}, c_{w_{l}}\right)=\operatorname{dist}\left(c_{w_{l}}, q_{l}\right)$. Clearly, $s_{j}^{\prime}=s_{j}$ if $v=1$ and $k=j-1, j$.

Proof of Theorem 5.1. We work in the settings of 5.2 and proceed by induction on $u$ and then, by induction on the number of intersections of the noncoinciding segments provided by $P^{\prime}$ with those provided by $P$. As we do not count as intersections the points $p, c_{1}, \ldots, c_{n}$, the intersections in question are represented by points in the interior of $D$ or in the interiors of the sides of $P$.

The case $u=0$ was done in 5.2. Therefore, we assume $u \geqslant 1$. This means that there exists $1 \leqslant j \leqslant n$ such that $s_{j}^{\prime} \neq s_{j}$. Hence, we obtain $v \geqslant 1$ disjoint geodesic segments as in 5.2.

We claim that the $2 n$-gons $P m_{k, w}$ and $P^{\prime}$ satisfy the induction hypothesis, where $w:=w_{1}$ if $v>1$ and $w:=j$ if $v=1$.

Suppose that $v=1$. Since $s_{j}^{\prime} \neq s_{j}$, we conclude that $k \neq j-1, j$ (see 5.2) and that $m_{k, j}$ is indeed applicable. Moreover, when we pass from $P$ to $P m_{k, j}$, the segment $s_{j} \subset \Sigma$ is substituted by the segment $s_{j}^{\prime} \subset \Sigma$ and the rest of the segments provided by $P$ remains the same. In other words, $u$ diminishes.

Suppose that $v>1$. Then $k \neq w_{1}-1, w_{1}$ because the interior of $\left[q_{0}, q_{1}^{\prime}\right]=\left[p_{k}, q_{1}^{\prime}\right]$ lives in the interior of $D$. So, $m_{k, w}$ is applicable. When passing from $P$ to $P m_{k, w}$, we simply replace $s_{w}$ by the segment $s$ represented by $\left[c_{w}, q_{0}\right]$. So, $u$ cannot grow and in fact remains the same. Every intersection with $s$ can be seen as an intersection with $\left[c_{w}, q_{0}\right]$ living inside the interior of $D$. Looking more closely at the triangle with the vertices $q_{0}, q_{1}^{\prime}, c_{w}$, we understand that every intersection of some $s_{l}^{\prime}$ with $s$ can be seen as an intersection of $s^{\prime} \subset D$, one of the disjoint segments related to $s_{l}^{\prime}$ (as in 5.2), with $\left[c_{w}, q_{0}\right]$. The latter induces an intersection of $s^{\prime}$ with $\left[q_{1}^{\prime}, c_{w}\right]$ because $s^{\prime}$ and $\left[q_{0}, q_{1}^{\prime}\right]$ are disjoint: they are related to different segments provided by $P^{\prime}$. It remains to observe that the intersection corresponding to $q_{1}^{\prime}$ disappears when passing from $P$ to $P m_{k, w}$

\section{References}

[Ale] A. D. Alexandrov, Convex polyhedra, Springer Monographs in Mathematics. Springer-Verlag, Berlin, 2005. Translated from the 1950 Russian edition by N. S. Dairbekov, S. S. Kutateladze, and 
A. B. Sossinsky, with comments and bibliography by V. A. Zalgaller and appendices by L. A. Shor and Yu. A. Volkov

[AGr] S. Anan'in, C. H. Grossi, Coordinate-free classic geometries, Moscow Math. J. 11 (2011), no. 4, 633-655

[Ana1] S. Anan'in, E. C. B. Goncalves A hyperelliptic view on Teichmüller space. I, arXiv: 0709.1711

[Ana2] S. Anan'in, Reflections, bendings, and pentagons, arXiv: 1201.1582

[DM1] P. Deligne, G. D. Mostow, Monodromy of hypergeometric functions and non-lattice integral monodromy, Publ. Math. IHES, no. 63 (1986), 5-89

[DM2] P. Deligne, G. D. Mostow, Commensurabilities among lattices in PU $(1, n)$, Annals of Math. Studies 132 (1993)

[DPP] M. Deraux, J. R. Parker, J. Paupert, New non-arithmetic complex hyperbolic lattices, Invent. Mat. 203 (2016), no. 3, 681-771

[FaW] E. Falbel, R. A. Wentworth, On products of isometries of hyperbolic space, Topology and its Applications 156 (2009), 2257-2263

[GhP] S. Ghazouani, L. Pirio, Moduli spaces of flat tori with prescribed holonomy, arXiv: 1604.01812

[GMST] W. M. Goldman, G. McShane, G. Stantchev, S. P. Tan, Automorphisms of two-generator free groups and spaces of isometric actions on the hyperbolic plane, arXiv: 1509.03790v2

[Gol] W. M. Goldman, The modular group action on real SL(2)-characters of a one-holed torus, Geometry \& Topology 7 (2003), 443-486

[HRi] C. D. Hodgson, I. Rivin, A characterization of compact convex polyhedra in hyperbolic 3-space, Invent. Mat. 111 (1993), 77-111

[Kui] N. H. Kuiper, Hyperbolic 4-manifolds and tessellations, Publ. Math. IHES, no. 68 (1988), 47-76

[McR] D. B. McReynolds, Arithmetic lattices in $\mathrm{SU}(n, 1), 2015$,

http://www.its.caltech.edu/ dmcreyn/ComplexArithmeticI.pdf

[MPa] G. Mondello, D. Panov, Spherical metrics with conical singularities on a 2-sphere: angle constraints, arXiv: 1505.01994

[Thu] W. P. Thurston, Shapes of polyhedra and triangulations of the sphere, Geometry \& Topology Monographs 1 (1998), 511-549

[WaW] P. Waterman, S. Wolpert, Earthquakes and tessellations of Teichmüller space, TAMS 278 (1983), no. $1,157-167$

Departamento de Matemática, icmC, Universidade de São Paulo, Caixa Postal 668, 13560-970-SÃO CARLOS-SP, BRASIL

E-mail address: sasha_a@icmc.usp.br

Departamento de Matemática, iCMC, Universidade de São Paulo, Caixa Postal 668, 13560-970-SÃo CARLOS-SP, BRASIL

E-mail address: grossi@icmc.usp.br

Korea Institute for Advanced Study (KIAS)

85 Hoegiro Dongdaemun-gu, Seoul 02455, Republic of Korea

E-mail address: jjlee@kias.re.kr

Departamento de Matemática, iCMC, Universidade de São Paulo, Caixa Postal 668, 13560-970-SÃo CARLOS-SP, BRASIL

E-mail address: joao.reis.reis@usp.br 

CAPÍTULO

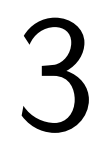

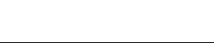

\section{REFERÊNCIA BIBLIOGRÁFICA}

[Ale] A. D. Alexandrov, Convex polyhedra, Springer Monographs in Mathematics. SpringerVerlag, Berlin, 2005. Translated from the 1950 Russian edition by N. S. Dairbekov, S. S. Kutateladze, and A. B. Sossinsky, with comments and bibliography by V. A. Zalgaller and appendices by L. A. Shor and Yu. A. Volkov

[AGr] S. Anan'in, C. H. Grossi, Coordinate-free classic geometries, Moscow Math. J. 11 (2011), no. $4,633-655$

[Ana1] S. Anan'in, E. C. B. Goncalves A hyperelliptic view on Teichmüller space. I, arXiv: 0709.1711

[Ana2] S. Anan'in, Reflections, bendings, and pentagons, arXiv: 1201.1582

[DM1] P. Deligne, G. D. Mostow, Monodromy of hypergeometric functions and non-lattice integral monodromy, Publ. Math. IHES, no. 63 (1986), 5-89

[DM2] P. Deligne, G. D. Mostow, Commensurabilities among lattices in PU(1,n), Annals of Math. Studies 132 (1993)

[DPP] M. Deraux, J. R. Parker, J. Paupert, New non-arithmetic complex hyperbolic lattices, Invent. Mat. 203 (2016), no. 3, 681-771

[FaW] E. Falbel, R. A. Wentworth, On products of isometries of hyperbolic space, Topology and its Applications 156 (2009), 2257-2263

[GhP] S. Ghazouani, L. Pirio, Moduli spaces of flat tori with prescribed holonomy, arXiv: 1604.01812

[GMST] W. M. Goldman, G. McShane, G. Stantchev, S. P. Tan, Automorphisms of two-generator free groups and spaces of isometric actions on the hyperbolic plane, arXiv: 1509.03790v2 
[Gol] W. M. Goldman, The modular group action on real SL(2)-characters of a one-holed torus, Geometry \& Topology 7 (2003), 443-486

[HRi] C. D. Hodgson, I. Rivin, A characterization of compact convex polyhedra in hyperbolic 3-space, Invent. Mat. 111 (1993), 77-111

[Kui] N. H. Kuiper, Hyperbolic 4-manifolds and tessellations, Publ. Math. IHES, no. 68 (1988), $47-76$

[McR] D. B. McReynolds, Arithmetic lattices in $S U(n, 1), 2015$, http://www.its.caltech.edu/ $\sim$ dmcreyn/ComplexArithmeticI.pdf

[MPa] G. Mondello, D. Panov, Spherical metrics with conical singularities on a 2-sphere: angle constraints, arXiv: 1505.01994

[Thu] W. P. Thurston, Shapes of polyhedra and triangulations of the sphere, Geometry \& Topology Monographs 1 (1998), 511-549

[WaW] P. Waterman, S. Wolpert, Earthquakes and tessellations of Teichmüller space, TAMS 278 (1983), no. 1, 157-167 


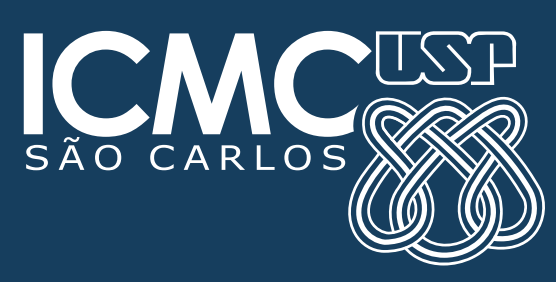

